The Palladio Component Model

Ralf Reussner, Steffen Becker, Jens Happe, Heiko Koziolek, Klaus Krogmann,

Michael Kuperberg

Interner Bericht 2007-21 



\section{The Palladio Component Model}

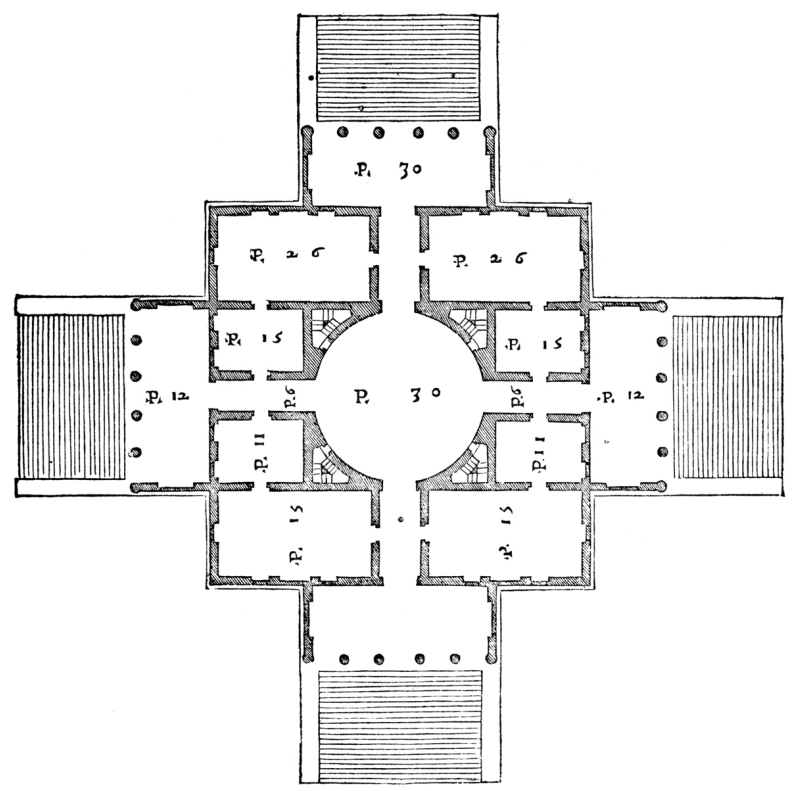

Ralf Reussner, Steffen Becker, Jens Happe,

Heiko Koziolek, Klaus Krogmann, Michael Kuperberg

Chair for Software Design \& Quality (SDQ)

University of Karlsruhe (TH), Germany

\footnotetext{
reussner@ipd.uka.de sbecker@ipd.uka.de happe@ipd.uka.de koziolek@ipd.uka.de

krogmann@ipd.uka.de mkuper@ipd.uka.de
}

October 8, 2007 


\section{Contents}

1 Introduction 4

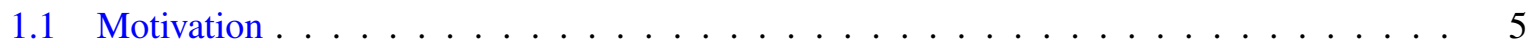

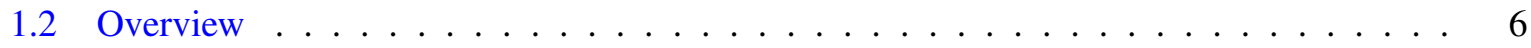

2 Foundations $\quad 7$

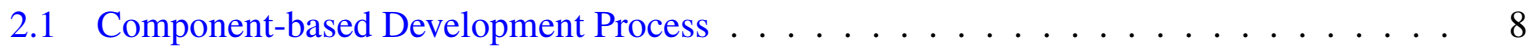

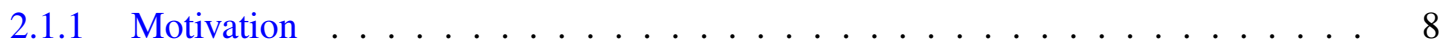

2.1.2 Roles in Component-based Development . . . . . . . . . . . . . . . . . 8

2.1.3 Development Process Model . . . . . . . . . . . . . . . . . . . . . . . . . 10

2.1 .4 Specification Workflow . . . . . . . . . . . . . . . . . . . . 12

2.1.5 QoS Analysis Workflow . . . . . . . . . . . . . . . . . . 14

2.1 .6 Discussion . . . . . . . . . . . . . . . . . . . . . 15

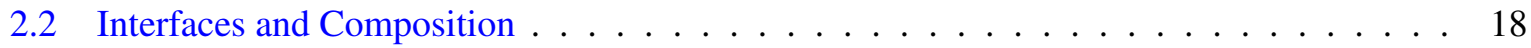

2.2.1 Interfaces as First-Class Entities . . . . . . . . . . . . . . . . . . 18

2.2 .2 Composed Structure . . . . . . . . . . . . . . . . . . . . . . . . . . 19

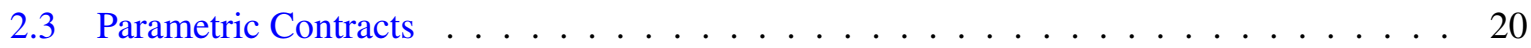

2.3.1 Classical Contracts for Software Components . . . . . . . . . . . . . . . . 20

2.3.2 Parametric contracts as a generalisation of classical contracts . . . . . . . . . 21

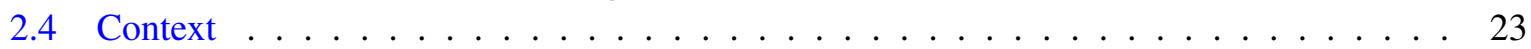

2.4 Motivation . . . . . . . . . . . . . . . . . 23

2.4 .2 Context Influences . . . . . . . . . . . . . . . . . . . . . . . 24

2.4 .3 An Explicit Context Model . . . . . . . . . . . . . . . . . . . . . . . . 26

2.5 Random Variables . . . . . . . . . . . . . . . . . . . . . . . 28

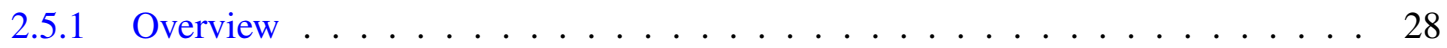

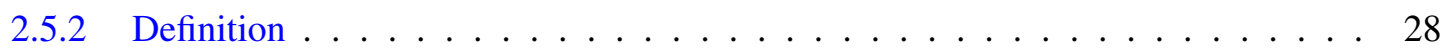

2.5 .3 PDF discretisation . . . . . . . . . . . . . . . . . . . . . 29

2.5 .4 Functional random variables $\ldots \ldots \ldots \ldots \ldots$

2.5 .5 Stochastic Expressions . . . . . . . . . . . . . . . . . 32

3 Concepts $\quad 35$

3.1 Component Developer $\ldots \ldots \ldots \ldots \ldots \ldots \ldots \ldots$

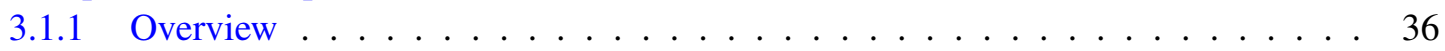

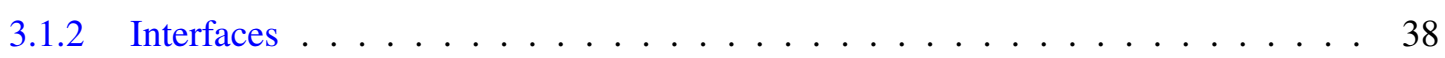

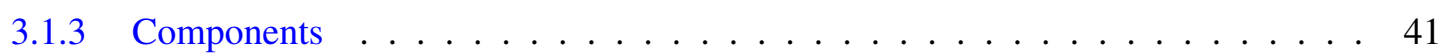

3.1 .4 Service Effect Specification . . . . . . . . . . . . . . . . . 47 


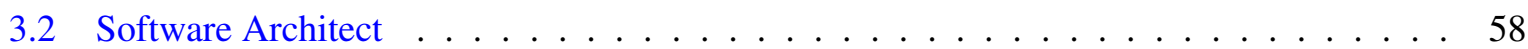

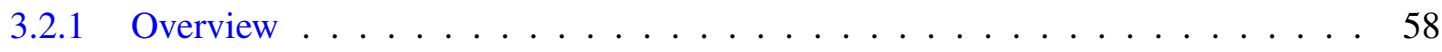

3.2 .2 Assembly . . . . . . . . . . . . . . . . . . . . . . . . 59

3.2 .3 Assembly Context . . . . . . . . . . . . . . . . . . . . . . . . 59

3.2 .4 System Assembly Connectors . . . . . . . . . . . . . . . . . . . . 60

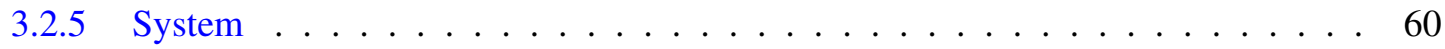

3.2 .6 System Roles . . . . . . . . . . . . . . . . . . . . . . . . . . 60

3.2 .7 System Delegation Connectors . . . . . . . . . . . . . . . . . . . . . . . . 60

3.3 System Deployer . . . . . . . . . . . . . . . . . . . . . . . . . 61

3.3 .1 Motivation . . . . . . . . . . . . . . . . . . . 61

3.3.2 Responsibilities of the Deployer . . . . . . . . . . . . . . . . . 61

3.3 .3 Resource Types . . . . . . . . . . . . . . . . . . . . . . 62

3.3.4 Resource Environment . . . . . . . . . . . . . . . . . . . . . . 63

3.3.5 Allocation Context . . . . . . . . . . . . . . . . . . . . 65

3.3 .6 Open Issues and Future Work _. . . . . . . . . . . . . . . . 67

3.4 Domain Expert . . . . . . . . . . . . . . . . . . . . . . . . . . . . . . 69

3.4 .1 Overview . . . . . . . . . . . . . . . . . . . . . . 69

3.4 .2 Usage Model . . . . . . . . . . . . . . . . . . . . . . . . . . 69

3.4 .3 Parameter Model . . . . . . . . . . . . . . . . . . . . . . 73

3.5 QoS Analyst . . . . . . . . . . . . . . . . . . . . . . 78

4 Technical Reference $\quad 79$

4.1 Core and Repository . . . . . . . . . . . . . . . . . . . . . . . . . . 80

4.2 Assembly and System . . . . . . . . . . . . . . . . . . . . . . . 84

4.3 Resource Type and Resource Environment . . . . . . . . . . . . . . . . . 85

4.4 Usage Model . . . . . . . . . . . . . . . . . . . . . . . . 86

5 Discussion $\quad 88$

$5.1 \quad$ PCM versus UML2 . . . . . . . . . . . . . . . . . . . . . . . . . . . . . . . . 89

5.2 Related Work . . . . . . . . . . . . . . . . . . . . . . . . . . . 91

5.3 Open Issues and Limitations $\ldots \ldots \ldots \ldots$

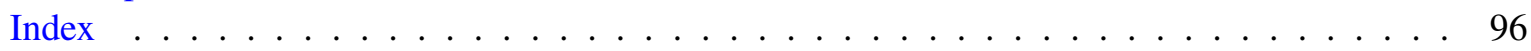


Chapter 1

\section{Introduction}




\subsection{Motivation}

This report introduces the Palladio Component Model (PCM), a novel software component model for business information systems, which is specifically tuned to enable model-driven quality-of-service (QoS, i.e., performance and reliability) predictions (based on work previously published in $[1,2,3$, $4,5])$. The PCM's goal is to assess the expected response times, throughput, and resource utilization of component-based software architectures during early development stages. This shall avoid costly redesigns, which might occur after a poorly designed architecture has been implemented. Software architects should be enabled to analyse different architectural design alternatives and to support their design decisions with quantitative results from performance or reliability analysis tools.

Component-based software engineering (CBSE) [6] promises many advantages over object-oriented or procedural development approaches. Besides increased reusability, better preparation for evolution, higher quality due to increased testing, and shorter time-to-market, CBSE potentially offers better predictability for the properties of architectures, because individual components should be provided with more detailed specifications. A large number of component models has been designed for different purposes. Component models used in the industry today (COM+/.NET, J2EE/EJB, CCM, etc.) do not offer capabilities for predicting QoS attributes. Component models from academia [7] have been designed to support purposes like runtime configuration, protocol checking, mobile device assessment etc. Some of them deal with QoS predictions (e.g., ROBOCOP, KLAPER, CB-SPE, PACC, etc.), but often have a different notion of software components.

Model-based QoS-prediction approaches for determining the performance and reliability of software systems have been researched for decades, but are still hardly used in practice. A survey by [8] classifies recent performance prediction approaches, and the overview by [9] includes a large number of reliability models. These approaches mostly target monolithic systems and are usually not sufficiently tuned for component-based systems. Specifying QoS properties of independently deployable software components is difficult, because component developers cannot know on what kind of machine their code is used, what parameters will be supplied to the component's provided services, and how the components required services will react.

Two key features of the PCM are i) the parameterised component QoS specification and ii) the developer role concept. Concerning i), the PCM is based on the component definition introduced by [6]. Software components are black box entities with contractually specified interfaces. They encapsulate their contents and are a unit of independent deployment. Most importantly, components can be composed with other components via their interfaces. The PCM offers a special QoS-specification for software components, which is parameterised over environmental influences, which should be unknown to component developers during design and implementation.

Concerning ii), the domain-specific language of the PCM is aligned to the different roles involved in component based development. Component developers specify models of individual components, which are composed by software architects to architectures. Deployers can model the hardware/VM/OSenvironment of the architecture, and domain experts are enabled to supply a description of the user's behaviour, which is necessary for QoS predictions. A QoS-driven development process model supports the roles in combining their models. 


\subsection{Overview}

This report is structured as follows:

- Chapter 2: lays the foundation to understand the concepts of the PCM. First, the QoS-driven development process model targeted by the PCM is introduced (2.1). Basic principles of interfaces and composition are highlighted. (2.2). Parametric contracts enable adapting the pre/postconditions of components (2.4). As QoS of component depends on the context a component is executed in, the PCM introduces a special context concept (2.4). To specify resource demands, random variables can be used in the PCM (2.5).

- Chapter 3: presents the concepts of the PCM and is structured after the roles involved in modeling. Component developers specify interfaces, services, components, and QoS properties (3.1). Software architects use the specifications of component developers to build architectures (3.2). Deployers model the resource environment of an architecture (3.3). Domain experts provide information about the user behaviour (3.4). QoS experts collect the information from the different roles, use prediction tools and pre-interpret the results (3.5).

- Chapter 4: contains the technical reference of the PCM.

- Chapter 5: discusses the PCM, describes related work, open issues, as well as limitations and assumptions present in the PCM. 
Chapter 2

\section{Foundations}




\subsection{Component-based Development Process}

\subsubsection{Motivation}

Component-based software development follows a different process than classical procedural or objectoriented development [6]. The task of developing software artefacts is split between the role of the component developer, who develops individual components, and the software architect, who assembles those components to form an application. Further roles are involved in specifying requirements and defining the resource environment.

For using the PCM, a specific development process with specific developer roles is envisioned, which builds on an existing component-based development process introduced by Cheeseman and Daniels [10], which was in turn based on the Rational Unified Process (RUP).

Early QoS analyses of a component-based architectures depend on information about its usage profile and resource environment. This information might not be available directly from software architects or component developers. Thus, further developer roles, such as deployers, domain experts and QoS analysts are needed for the specification and QoS analysis of a component-based architectures. These developer roles and their tasks are described in Section 2.1.2. For the PCM, a domain specific modeling language has been created for each of these roles. These modeling languages will be described in detail in Chapter 3.

The PCM process model extends the process model by Cheeseman and Daniels (Section 2.1.3). Section 2.1.4 elaborates on the specification workflow and illustrates the interdependencies between component developer and software architect. The PCM process model additionally contains a workflow "QoS-Analysis" (Section 2.1.5), in which all of the developer roles interact to predict the performance or reliability of the architecture.

The development process introduced in the following is generic, so that it could be followed by other model-based QoS prediction approaches for component systems [11] as well. It is furthermore not restricted to a specific QoS property like performance, but can also be used for reliability, availability, security, safety, etc. The process model reflects our vision of software development including early QoS analyses. Its applicability in practice remains to be validated. Some discussion points about the process model as well as related work are summed up in Section 2.1.6.

\subsubsection{Roles in Component-based Development}

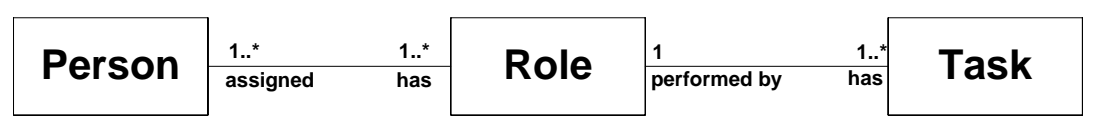

Figure 2.1: Concept of Roles.

Before introducing the individual roles of the component-based development process, we describe the general concept of roles in software development. Figure 2.1 illustrates the relation of persons, roles, and tasks. A role groups a set of tasks that have an overall purpose and each task is associated to exactly one role. For example, the role component developer performs tasks like component implementation and component specification. A role can be adopted by multiple persons, e.g. there can be multiple persons who are component developers involved in the process. On the other hand, it is also possible for a person to adopt multiple roles. For instance, some component developers might also play the role of software architects, who are responsible for designing the software architecture. The relation of persons and roles 
is an important concept and has to be considered when reading the following descriptions of roles.

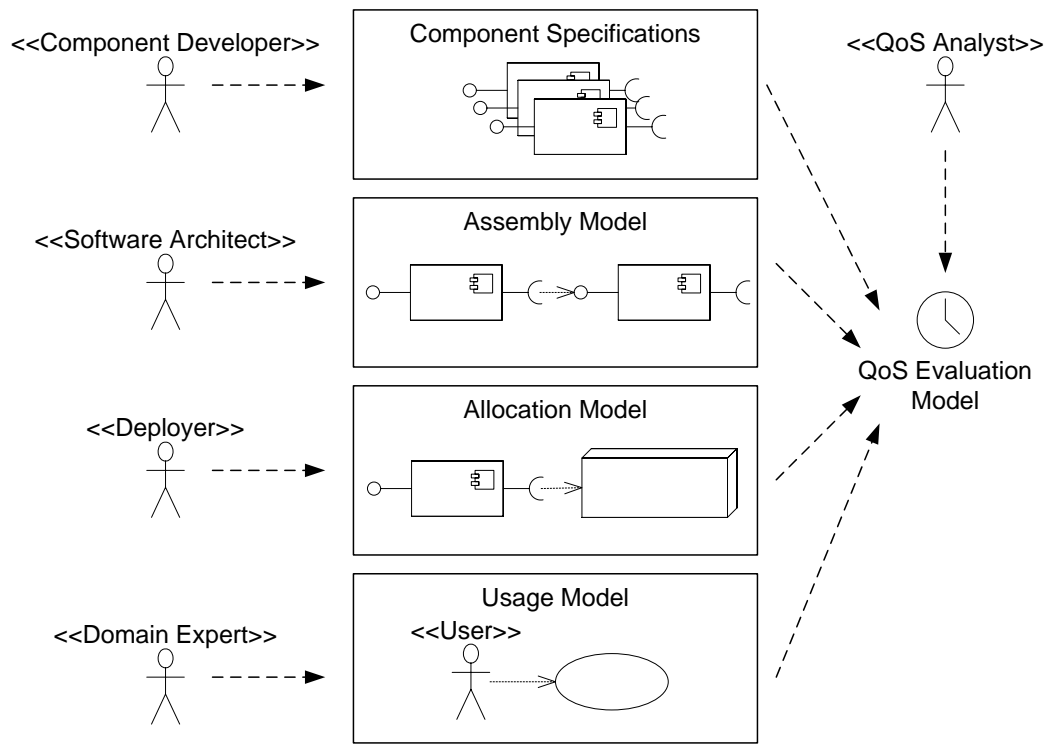

Figure 2.2: Developer Roles in the Palladio Process Model

Since we want to evaluate QoS attributes during early development stages, we need additional information about the internal component structure, the usage model, and the execution environment. These cannot be provided by a single person (e.g., the software architect) involved in the development process, since the required knowledge is spread among different persons with different development roles. Therefore, a QoS analyst requires support of component developers, software architects, domain experts, and deployers to analyse the QoS attributes of a software architecture (cf. Fig. 2.2).

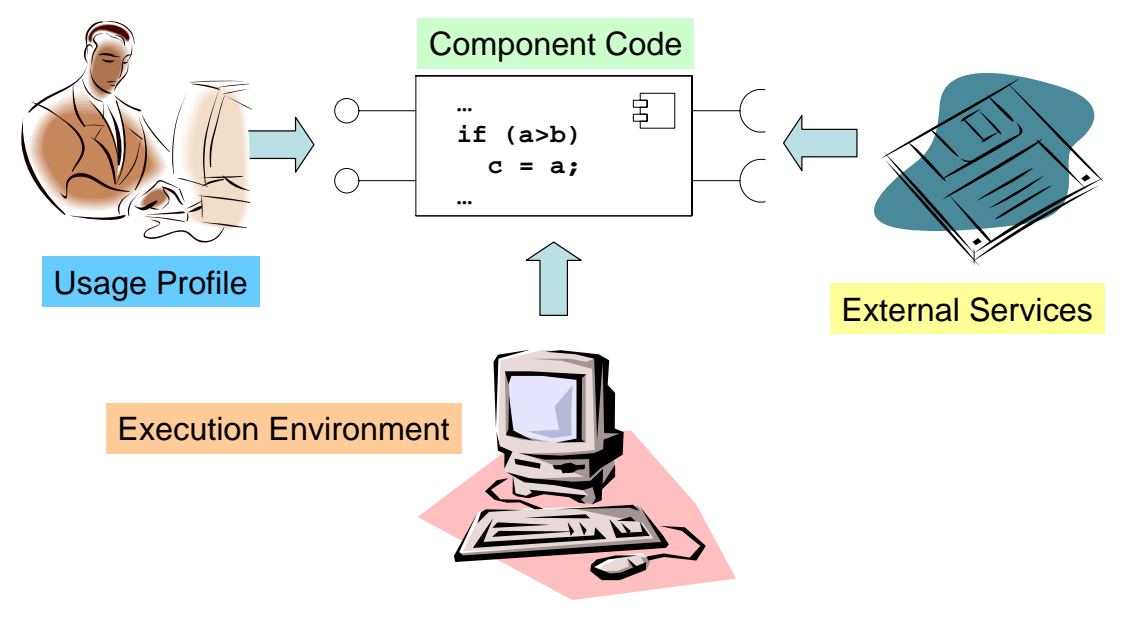

Figure 2.3: Influences on the QoS properties of a Software Component

Component developers are responsible for the specification and implementation of components. They develop components for a market as well as per request. To enable QoS analyses, they need to specify the QoS properties of their components without knowing a) to which other components they are connected, b) on which hardware/software platform they are executed, and c) which parameters are used 
when calling their services (cf. Figure 2.3). Only such a specification enables independent third party analyses. In the PCM, component developers use service effect specifcations (SEFF, cf. Section 3.1.4) to characterise the QoS properties of their components.

Software architects lead the development process for a complete component-based application. They design the software architecture and delegate tasks to other involved roles. For the design, the planned application specification is decomposed into component specifications. Existing component specifications can be selected from repositories to plan the integration of existing components into a software architecture. If no existing specification matches the requirements for a planned component, a new component has to be specified abstractly. The software architect can delegate this task to component developers. Additionally, the software architect specifies component connections thereby creating an assembly model (cf. Section 3.2.2). After finishing the design, software architects are responsible for provisioning components (involving make-or-buy decisions), assembling component implementations, and directing the tests of the complete application.

Deployers specify the resources, on which the planned application shall be deployed. Resources can be hardware resources, such as CPUs, storage devices, network connections etc., as well as software resources, such as thread pools, semaphores or database connection. The result of this task is a so-called resource environment specification (cf. Section 3.3.4). With this information, the platform independent resource demands from the component specifications can be converted into timing values, which are needed for QoS analyes. For example, a component developer may have specified that a certain action of a component service takes 1000 CPU cycles. The resource environment specification of the deployer provides the information how many cycles the CPU executing the component processes per second. Both information together yield the execution time of the action. Besides resource specification, deployers allocate components to resources. In the PCM, this step can also be done during design by creating a so-called allocation model (cf. Section 3.3.5). Later in the development process, during the deployment stage, deployers can be responsible for the installation, configuration, and start up of the application.

Domain experts participate in requirement analysis, since they have special knowledge of the business domain. They are familiar with the users' work habits and are therefore responsible for analysing and describing the user behaviour. This includes specifying workloads with user arrival rates or user populations and think times. In some cases, these values are already part of the requirement documents. If method parameter values have an influence on the QoS of the system, the domain experts may characterise these values. The outcome of the domain experts' specification task is a so-called usage model (cf. Section 3.4.2).

QoS analysts collect and integrate information from the other roles, extract QoS information from the requirements (e.g., maximal response times for use cases), and perform QoS analyses by using mathematical models or simulation. Furthermore, QoS analysts estimate missing values that are not provided by the other roles. For example, in case of an incomplete component specification, the resource demand of this component has to be estimated. Finally, they assist the software architects to interpret the results of the QoS analyses.

\subsubsection{Development Process Model}

In the following, the roles described in the former section are integrated into a development process model featuring QoS analysis. We focus on the development process that is concerned with creating a working system from requirements and neglect the concurrent management process that is concerned with time planning and controlling. We base our model on the UML-centric development process model described by Cheeseman and Daniels [10], which is itself based on the Rational Unified Process (RUP). 


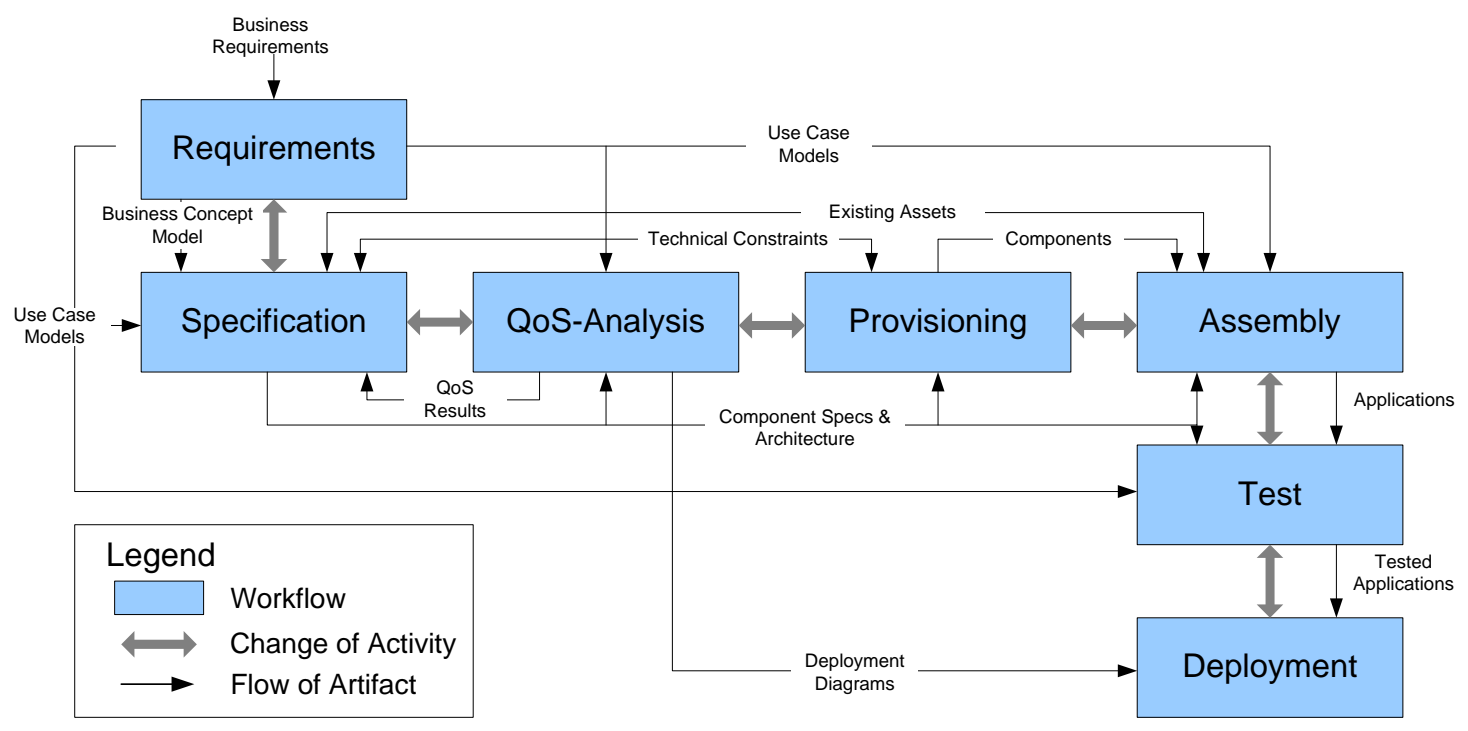

Figure 2.4: QoS Driven Process Model: Overview

The main process is illustrated in Figure 2.4. Each box represents a workflow. Thick arrows between boxes represent a change of activity, while the thin arrows characterise the flow of artifacts between the workflows. The workflows do not have to be traversed sequentially (i.e., no waterfall model). Backward steps into former workflows are allowed. The model also allows an incremental or iterative development based on prototypes.

The workflows requirements, provisioning, assembly, test, and deployment have mainly been inherited from the original model and will briefly be described in the following. The workflow "specification" has been slightly modified to explicitly include the interaction between component developer and software architect and the specification of extra-functional properties. The workflow "QoS Analysis" has been added to the model and will be described in detail below.

Requirements The business requirements coming from customers are formalised and analysed during this workflow. It produces a business concept model and a use case model. The former is a conceptual model of the business domain and creates a common vocabulary between customers and developers. It is, however, not relevant for QoS Analysis. The latter describes the interaction between users (or other external actors) with the system. It establishes the system boundaries and a set of use cases that define the functional requirements.

Specification Business concept model and use case model are input from the requirements to this workflow. Additionally, technical constraints, which might have been revealed during provisioning, and QoS metrics from already performed QoS predictions can be input to the specification workflow after initial iterations of the process model. During specification, the component-based software architecture is designed. Components are identified, specified, and their interaction is defined. The software architect usually interacts with component developers during this workflow. More detail about this workflow is provided in Section 2.1.4. The output artifacts of this workflow are complete component specifications (in PCM also extra-functional specifications) and the component architecture (called assembly model in the PCM). 
QoS Analysis Component specifications, the architecture, and use case models are input to the QoS analysis workflow. During this workflow, deployers provide models of the resource environment of the architecture, which contain specifications of extra-functional properties (Section 3.3). The domain expert takes the use case models, refines them, and adds QoS-relevant information, thereby creating a PCM usage model (Section 3.4). Finally, the QoS-Analyst a) combines all of the models, b) estimates missing values, c) checks the models' validity, d) feeds them into QoS predictions tools, and e) prepares a pre-evaluation of their predictions, which is targeted at supporting the design decisions of the software architect. More detail about the QoS analysis workflow follows in Section 2.1.5. Outputs of the QoS analysis are pre-evaluated results for QoS metrics, which can be used during specification to adjust the architecture, and deployment diagrams that can be used during deployment.

Provisioning Compared to classical development processes the provisioning workflow resembles the classical implementation workflow. However, one of the assets of component-based development is reuse, i.e. the incorporation of components developed by third parties. During the provisioning workflow "make-or-buy" decisions are made for individual components. Components that cannot be purchased from third-parties have to be implemented according to the specifications from the corresponding workflow. Consequently, the provisioning workflow receives the component specifications and architecture as well as technical constraints as inputs. The outputs of this workflow are implemented software components.

Assembly Components from the provisioning workflow are used in the assembly workflow. Additionally, this workflow builds up on the component architecture and the use case model. The components are assembled according to the assembly model during this workflow. This might involve configuring them for specific component containers or frameworks. Furthermore, for integrating legacy components it might be necessary to write adapters to bridge unfitting interfaces. The assembled components and the complete application code are the outputs of this workflow.

Test The complete component-based application is tested according to the use case models in this workflow in a test environment. It also includes measuring the actual extra-functional properties of the application and their comparison with the predicted values. Once the functional properties have been tested and the extra-functional properties are satisfiable in the test environment the application is ready for deployment in the actual customer environment.

Deployment During deployment, the tested application is installed in its actual customer environment. The term deployment is also used to denote the process of putting components into component containers, but here the term refers to a broader task. Besides the installation, it might be necessary to adopt the resource environment at the customer's facilities or to instruct future users of the system. For the mapping of components to hardware resources, the deployment diagrams from the QoS analysis workflow can be used.

\subsubsection{Specification Workflow}

The specification workflow (see Figure 2.5, right column) is carried out by software architects. The workflows of the software architect and the component developers influence each other. Existing components (e.g., from a repository) may have an impact on the inner component identification and component specification workflow, as the software architect can reuse existing interfaces and specifications. Vice versa, 
components newly specified by the software architect serve as input for the component requirements analysis of component developers, who design and implement new components.

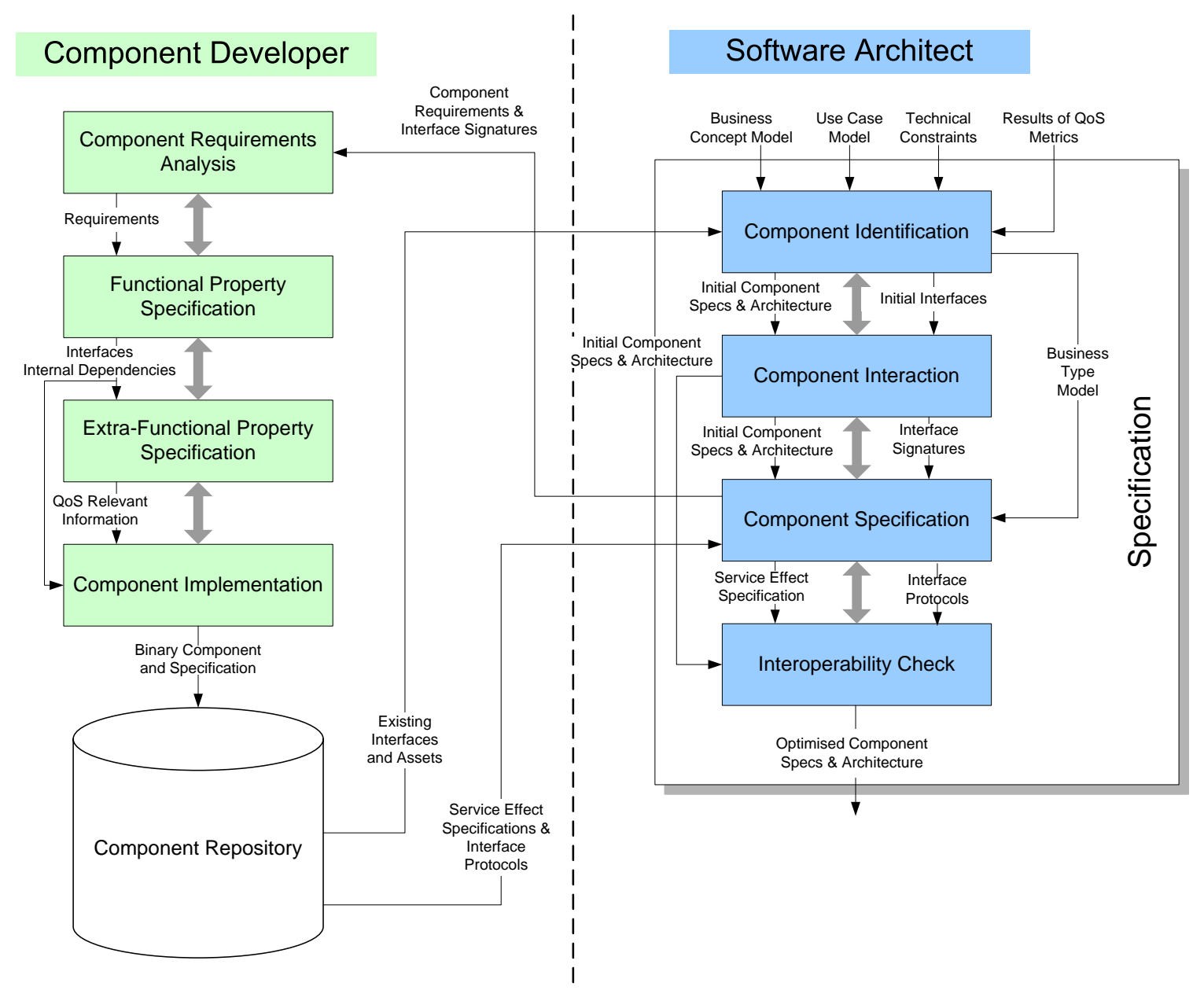

Figure 2.5: Specification Workflow

The component developer's workflow is only sketched here, since it is performed separately from the software architect's workflows. If a new component needs to be implemented, the workflow of the component developer (see Figure 2.5) can be assumed to be part of the provisioning workflow according to Cheesman and Daniels [10].

Any development process model can be used to construct new components as long as functional and extra-functional properties are specified properly. First, a component requirements analysis has to be conducted. It is succeeded by functional property specification and then extra-functional property specification. The functional properties consist of interface specifications (i.e., signatures, pre/postconditions, protocols), descriptions of internal dependencies between provided and required interfaces. We use service effect specifications (Section 3.1.4) to describe such dependencies. Additionally, descriptions of the functionality of component services have to be made. Extra-functional, QoS-relevant information includes resource demands, reliability values, data flow, and transition probabilities for service effect specifications. Finally, after component implementation according to the specifications, component developers have to put the binary implementations and the specifications into repositories, where they can 
be retrieved and assessed by software architects.

The specification workflow of the software architect consists of four inner workflows. The first two workflows (component identification and component interaction) are adapted from [10] except that we explicitly model the influence on these workflows by existing components. For component identification, so-called ProvidedComponentTypes can be used in the PCM (cf. Section 3.1.3.3). Component interaction can be described in the PCM once the provided component types have evolved to ImplementationComponent Types (cf. Section 3.1.3.1). During the component specification, the software architect additionally gets existing interface and service effect specifications as input. Both are transferred to the new workflow interoperability check. In this workflow, interoperability problems are solved and the architecture is optimised. For example, functional parametrised contracts [12], which are modelled as service effect specifications, can be computed (cf. Section 2.3). The outputs of the specification workflow are an architecture and component specifications with refined interfaces.

\subsubsection{QoS Analysis Workflow}

During QoS analysis, the software architecture is refined with information on the deployment context, the usage model, and the internal structure of components. Figure 2.6 shows the process in detail.

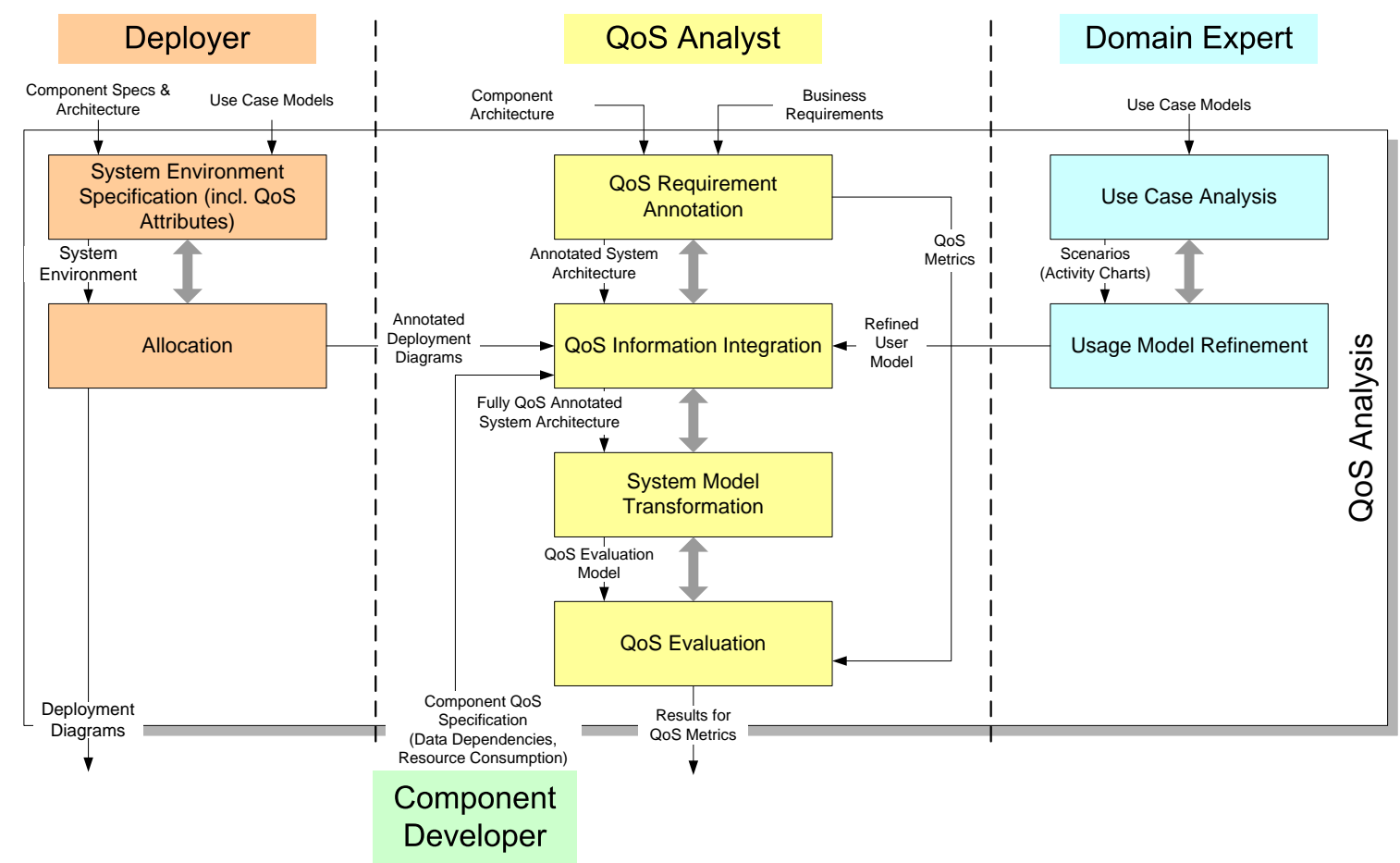

Figure 2.6: Detailed View of the QoS Analysis Workflow

The deployer starts with the resource environment specification based on the software architecture and use case models. Given this information, the required hardware and software resources and their interconnections are derived. As a result, this workflow yields a description of the resource environment, for example, a deployment diagram without allocated components or an instance of the resource environment model (cf. Section 3.3.4). Instead of specifying a new resource environment, the deployer can also use the descriptions of existing hardware and software resources. Moreover, a set of representative sys- 
tem environments can be designed if the final resource environment is still unknown. For QoS analysis, detailed information on the resources modelled in the environment are required.

During allocation, the deployer specifies the mapping of components to resources. The result of this workflow can be a complete deployment diagram or a resource environment plus allocation contexts for components as described in section 3.3.5. The resulting specifications are part of the inputs of the QoS analysis models used later. The resulting fully specified resource environment and component allocation are passed to the QoS analyst.

The domain expert refines the use case models based on the requirements during the use case analysis workflow. A description of the scenarios for the users is created based on an external view of the current software architecture. The scenarios describe how users interact with the system and which dependencies exists in the process. For example, activity charts or usage models (cf. Section 3.4.2) can be used to describe such scenarios. The scenario descriptions are input to the usage model refinement. The domain expert annotates the descriptions with, for example, branching probabilities, expected size of different user groups, expected workload, user think times, and parameter characterisations.

As the central role in QoS analysis, QoS analysts integrate relevant information, perform evaluations, and deliver feedback to all involved parties. In the QoS requirement annotation workflow, the QoS analyst maps QoS requirements to direct requirements of the software architecture. For example, the maximum waiting time of a user becomes the upper limit of the response time of a component's service. While doing so, the QoS analyst selects metrics, like response time or probability of failure on demand, that are evaluated during later workflows.

During QoS information integration, the QoS analyst collects the specifications provided by the component developers, deployers, domain experts, and software architects, checks them for soundness, and integrates them into an overall QoS model of the system. In case of missing specifications, the QoS analyst is responsible for deriving the missing information by contacting the respective roles or by estimation and measurement. The system specification is then automatically transformed into a prediction model.

The QoS evaluation workflow either yields an analytical or simulation result. QoS evaluation aims, for example, at testing the scalability of the architecture and at identifying bottlenecks. The QoS analyst performs an interpretation of the results, comes up with possible design alternatives, and delivers the results to the software architect. If the results show that the QoS requirements cannot be fulfilled with the current architecture, the software architect has to modify the specifications or renegotiate the QoS requirements.

\subsubsection{Discussion}

Related Work There are numerous publications on component-based development processes $[6,13$, $14,15,16,2]$. However, most of these process descriptions do not deal with extra-functional properties. Furthermore, there are many approaches for the performance prediction of component-based software systems [11], but only few describe the encompassing development process in detail or spread the needed information for QoS analyses among the participating roles.

Role Names There are several synonyms for the role names we have chosen for the PCM process model.

- Component Developer: Application Component Provider, Component Implementer, Component Programmer 
We chose "component developer" because it is quite generic, should be known to most software engineers, and describes the tasks of this role best.

- Software Architect: System Architect, Component Assembler, System Assembler, Architect, Application Assembler

It might be argued that this role does not only deal with software, but also has an influence on the hardware environment. Therefore the broader term 'system' instead of 'software' could be used. However, the term software architect is quite established and the tasks involving the hardware environment of the component-based system could be delegated to the deployer. The term component assembler is sometimes used in the literature, it is, however, a too restricted term for the tasks of this role.

- Deployer: System Allocator, Component Deployer, Assembly Allocator, System Administrator, Resource Specifier, Execution Environment Modeller, Middleware Expert, Deployment Expert In J2EE the term 'deployment' is used for assembling components and allocating them on resources. In the PCM, we explicitly separate between assembly and allocation, as the former is conducted by the software architect and the latter by the deployer. We chose the most generic term 'deployer' for the role, which is responsible for specifying the resource environment and allocating component assemblies to resources.

- QoS Analyst: Performance Analyst, Reliability Specialist, QoS Expert, QoS Evaluator, QoS Manager

As we do not want to restrict our model to performance or reliability analyses we chose the collective term 'QoS' (Quality-of-Service), which covers performance, reliability, availability, etc. The goal of this role is to come up with analyses of the QoS properties of an application, so we chose the term 'QoS analyst'

- Domain Expert: Business Expert, Usage Modeller

We are not sure, if there are dedicated roles for specifying user behaviour in IT organisations. Therefore we chose the term 'domain expert', because this role might be involved into other tasks related to requirements analyses in addition to the task of usage modelling.

Is there a QoS Analyst? As described in Section 2.1.2, the role of QoS analysts bundles the tasks of 1) deriving QoS information from the requirements, 2) integrating information from the other roles, 3) estimating missing input parameters, 4) using QoS analysis tools such as queueing network solver, and 5) pre-interpreting the results of these tools.

It can be argued that this role is not really necessary, as most of the tasks could also be performed by the software architect. In fact, task 2), 4), and 5), should even be encapsulated into user-friendly tools, so that no special knowledge would be required to perform the QoS analysis. Task 3) might require special knowledge of a QoS domain, but software architects should at least be able to provide rough estimation for missing values, which might be sufficient for early QoS analysis.

However, it can also be argued, that existing tools are not so far advanced to automate the tasks of this role. The manual specification of additional input parameters for the prediction method might be too time-consuming and thus expensive for software architects. Additionally, it remains questionable if task 5) can be encapsulated into tools as it is sometimes difficult to map analysis or simulation results to problems in the architecture. Furthermore, designing QoS-improving architectural alternatives requires special knowledge (such as performance patterns or configuration options of component containers). 
We have decided to keep the role in the model, because of role-based separation of concerns. We suppose that today the QoS analysis task is often delegated to specialists. 


\subsection{Interfaces and Composition}

\subsubsection{Interfaces as First-Class Entities}

According to Parnas [17], an interface is an abstraction of piece of software (a software entity) which should contain a sufficient amount of information for a caller to understand and finally request the realised functionality from any entity claiming to offer the specified functionality. Note that this implies, that the specification of the interface also has to contain a sufficient amount of information for the implementer to actually implement the interface. Due to the inherent need of an interface to abstract the behaviour of the software entity not in all cases there is sufficient information provided to use or implement an interface in an unambiquious way.

This definition has several consequences. First of all, interfaces can exist on their own, i.e., without any entity requesting or implementing the specified functionality. In industrial practice, this is actually often used. For example, Sun defined for the Java programming language several sets of Java interfaces which deal with a specific sets of generic functionality without actually providing an implementation. Part of these domain-standards are the Java Messaging Standard dealing with different types of message-based communication or the Java Persistence API concerned with persisting objects in relational databases.

Second, two roles can be identified a software entity can take relative to an interface. Any entity can either claim to implement the functionality specified in an interface or to request that functionality. This is reflected in the Palladio Component Model by a set of abstract meta-classes giving a conceptual view on interfaces, entities and their relationships. The abstract meta-class InterfaceProvidingEntity is inherited by all entities which can potentially offer interface implementations (Figure 2.7). Similarly, the meta-class InterfaceRequieringEntity is inherited by all entities which are allowed to request functionality offer by entities providing this functionality. Details follow in Section 3.1.

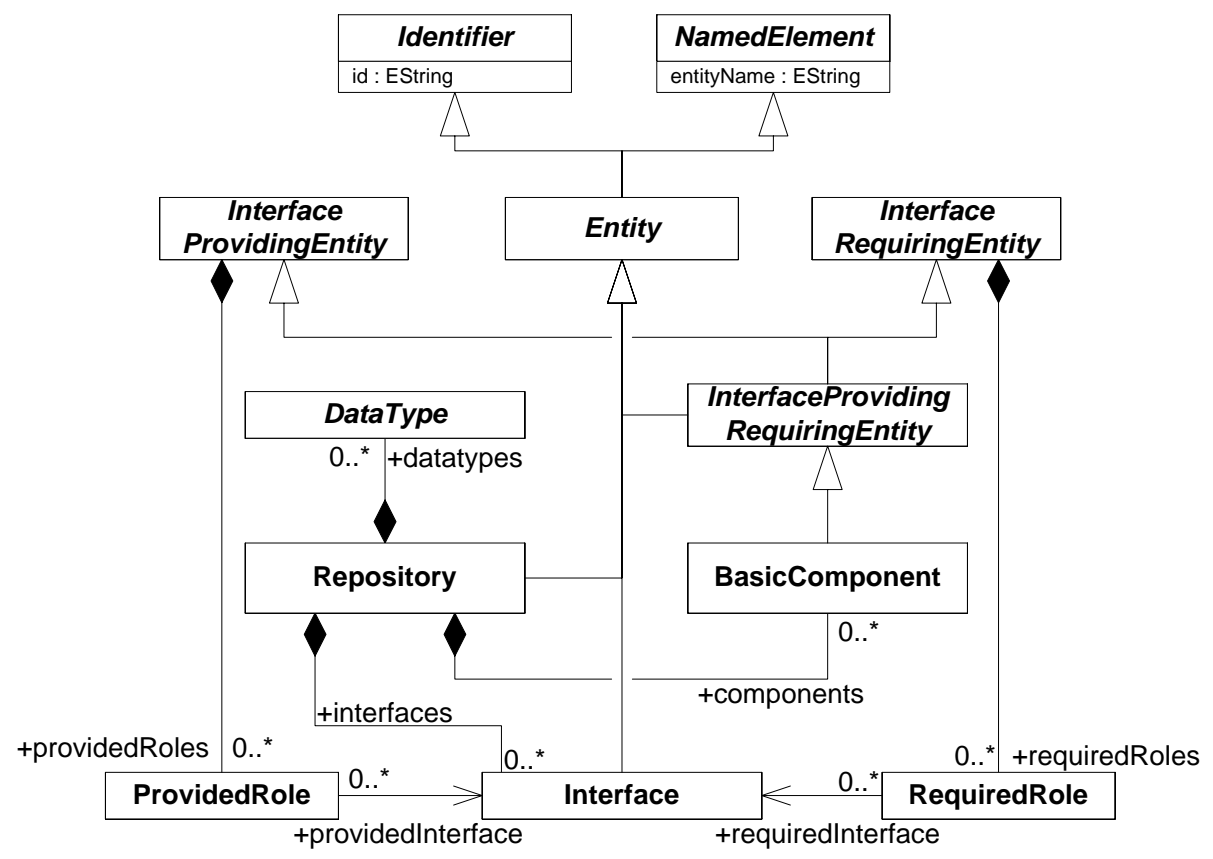

Figure 2.7: InterfaceProvidingRequiringEntity in the PCM metamodel 


\subsubsection{Composed Structure}

Clements Szyperski approaches the definition of a component in his book on component based softwareengineering with the statement "Components are for composition, much beyond is unclear" [6]. This statement does not only point out how hard it is to find a common definition for the term "component". It also highlights the most common principle in the definitions of the term component in the literature: a component can be composed with other components in order to get a more complex structure. We also consider the ability to compose components into new structures as a primary feature of components. As a consequence, our component model contains an abstract conceptual view of the concept of a ComposedStructure, which is a structure build by composing components (Figure 2.8, details in Section 3.2).

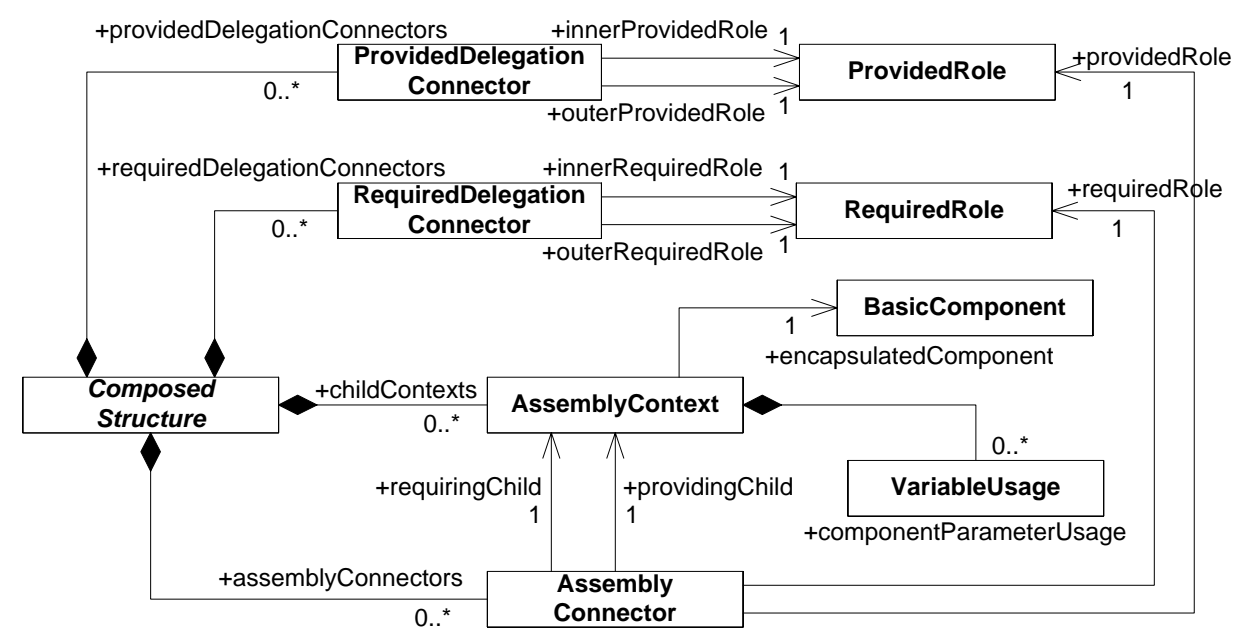

Figure 2.8: ComposedStructure in the PCM metamodel 


\subsection{Parametric Contracts}

\subsubsection{Classical Contracts for Software Components}

Before defining contracts for components, we briefly review B. Meyer's design-by-contract principle from an abstract point of view. According to [18, p. 342], a contract between the client and the supplier consists of two obligations:

- The client has to satisfy the precondition of the supplier.

- The supplier has to fulfill its postcondition, if the precondition was met by the client.

Each of the above obligations can be seen as the benefit for the other party. (The client can count on the postcondition if the precondition was fulfilled, while the supplier can count on the precondition). Putting it in one sentence:

If the client fulfills the precondition of the supplier, the supplier will fulfil its postcondition.

The used component plays the role of a supplier. But to formulate contracts for components, we also have to identify the pre- and postconditions and the user of a component. But what is to be considered a precondition, postcondition and user depends on whether the component is used at run-time or configuration-time. Let's first consider the component's use at run-time. Using a component at run-time is calling its services. Hence, the user of a component $C$ is the set of all components connected to $C$ 's provides-interface(s).

The precondition for that kind of use is the precondition of the service, likewise the postcondition is the postcondition of the service. Actually, that shows that this kind of use of a component is nothing different as using a method. Therefore, the author considers this case as the use of a component service, but not as the use of a component. Likewise, the contract to be fulfilled here from client and supplier is a method contract as described by B. Meyer already in 1992. This is the contract for using a component service, but not the contract for using the component!

The other case of component usage (usage at composition-time) is actually the relevant case when talking about the contractual use of components. This is the important case when architecting systems out of components or deploying components within existing systems for reconfigurations. Again, in this case a component $C$ is acting as a supplier and the component connected to the provides interface(s) as a client. The component $C$ offers services to the those components of the assembly context which are connected to $C$ 's provides-interface(s). According to the above discussion of contracts, these offered services are the postcondition of the component, i.e., what the client can expect from a working component. Also according to B. Meyer's above mentioned description of contracts, the precondition is what the component $C$ expects from those components of the assembly context which are connected to $C$ 's requires-interface(s) to be provided by the assembly context, in order to enable $C$ to offer its services (as stated in its postcondition). Hence, the precondition of a component is stated in its requires-interfaces. Analogously to the above single sentence formulation of a contract, we can state:

If the user of a component fulfills the component's requires-interface (offers the right required components in the assembly context) the component will offer its services as described in the provides-interface.

Let us denote with pre $_{c}$ the precondition of a component $c$ and with post $t_{c}$ the postcondition of a component $c$. For checking whether a component $c$ can be replaced safely by a component $c^{\prime}$, one has to ensure 
that the contract of $c^{\prime}$ is a subcontract of $c$. The notion of a subcontract is described in [18, p. 573] like contravariant typing for methods: A contract $c^{\prime}$ is a subcontract of contract $c$ iff

$$
\text { pre }_{c^{\prime}} \unlhd \text { pre }_{c} \wedge \text { post }_{c^{\prime}} \unrhd \text { post }_{c}
$$

(Where $\unrhd$ means "stronger", i.e., if pre $_{c}$ and post $_{c}$ are predicates, $\unrhd$ is the logical implication $\Rightarrow$. In the set semantics of pre- and postcondition below, $\unrhd$ is the inclusion $\supseteq$.)

To check the interoperability between components $c$ and $c^{\prime}$ (see point (1) in figure 2.9), one has to check whether

$$
\text { pre }_{c} \unlhd \text { post }_{c^{\prime}}
$$

Coming back to protocol-modelling interfaces, we can consider the precondition of a component as the set of required method call sequences, while the postcondition is the set of offered call sequences. In this case, the checks described in the above formulas (2.1) and (2.2) boiled down to checking the inclusion relationship between the sets of call sequences, i.e., for the substitutability check we have:

$$
\text { pre }_{c^{\prime}} \subseteq \text { pre }_{c} \wedge \text { post }_{c^{\prime}} \supseteq \text { post }_{c}
$$

and for the interoperability check:

$$
\text { pre }_{c} \subseteq \text { post }_{c^{\prime}}
$$

For arbitrary sets $A$ and $B$ holds $A \subseteq B \Longleftrightarrow A \cap B=A$. Hence, the inclusion check we need for checking interoperability and substitutability can be reduced to computing the intersection and equivalence of sets of call sequences. One of the main reasons for choosing finite state machines (FSMs) as a model to specify these sets of call sequences was the existence of efficient algorithms for computing the intersection of two FSMs and checking their equivalence. Of course, more powerful models than FSMs exist (in the sense that they can describe protocols which cannot be described by FSMs) but for many of these models (like the various push-down automata) the equivalence is not decidable (see e.g., [19]). Hence, one can use these models for specifying component interfaces, but that does not help to check their interoperability or substitutability at configuration-time.

\subsubsection{Parametric contracts as a generalisation of classical contracts}

While interoperability tests check the requires-interface of a component against the provides-interface of another component, parametric contracts link the provides-interface of one component to the requiresinterface of the same component (see points (2) and (3) in figure 2.9).

The usefulness of parametric contracts is based on the observation that in practice often only a subset of a component's functionality is used. This is especially true for coarse-grained components. In this case, also only a subset of the functionality described in the requires-interface is actually used. That means that the component could be used without any problems in assembly contexts where not all dependencies, as described in the requires interface, are fulfilled. Vice versa, if a component does not receive all (but some) functionality it requires from the assembly context, it often can deliver a reasonable subset of its functionality.

These facts can be modelled by a set of possible provides-interfaces $\mathbf{P}:=\{$ prov $\}$ and a set of possible requires-interfaces $\mathbf{R}:=\{r e q\}$ and a monotone total bijective mapping $p$ between them $p$ : $\mathbf{P} \rightarrow \mathbf{R} .{ }^{1}$ As a result, each requires-interface $r e q \in \mathbf{R}$ is now a function of a provides-interface prov:

\footnotetext{
${ }^{1} p$ can be made total and surjective by defining $\mathbf{P}:=\operatorname{dom}(p)$ and $\mathbf{R}:=i m(p)$.
} 


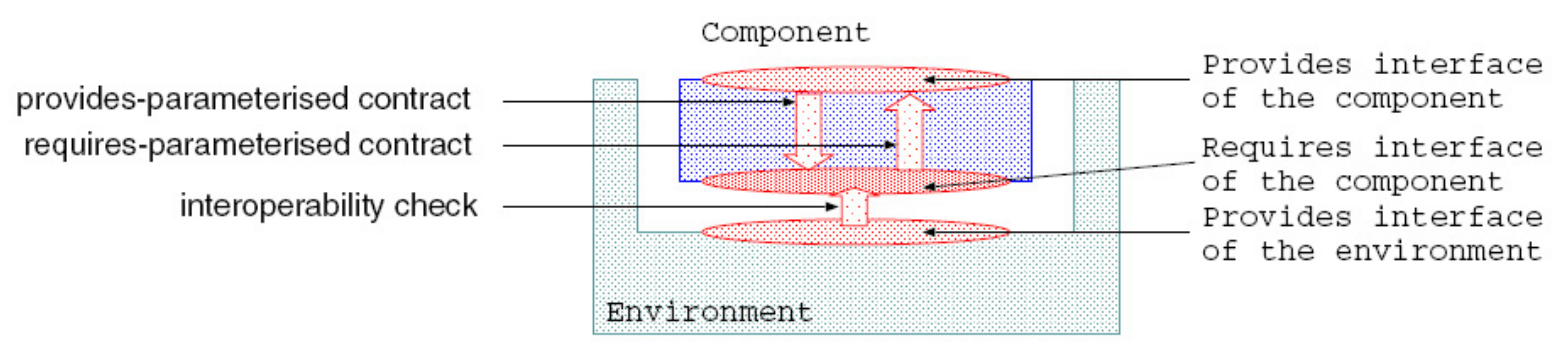

Figure 2.9: Interoperability checks (1) and Requires-parametric Contract (2) and Provides-parametric Contract (3)

$r e q=p($ prov $)$ and (because $p$ is bijective) each provides-interface prov $\in \mathbf{P}$ can be modelled as a function of a requires-interface $r e q \in \mathbf{R}:$ prov $=p^{-1}(r e q)$.

This mapping $p$ is now called parametric contract, since it parameterises the precondition with the postcondition of the component and vice versa. It can be considered as a generalisation of "classical contract" which uses a fixed pre- and postcondition. The parametric contract is bundled with the component and computes the interfaces of the components on demand.

For the following, assume component $B$ uses component $C$ and is used by component $A$. If component $A$ uses only a subset of the functionality offered by $B$ we compute a new requires-interface of $B$ with the parametric contract $p_{B}$ :

$$
p_{B}\left(r e q_{A} \cap \operatorname{prov}_{B}\right)=: r e q_{B}^{\prime} \subseteq r e q_{B}
$$

Note that the new requires-interface $r e q_{B}^{\prime}$ requires possibly less than the original requires-interface $r e q_{B}:=p_{B}\left(\operatorname{prov}_{B}\right)$ (but never more) since $p_{B}$ is monotone and $\operatorname{req}_{A} \cap$ prov $_{B} \subseteq$ prov $_{B}$. When computing the requires-interface out of a provides-interface (possibly intersected with an external requiresinterface) the parametric contract is called provides-parametric contract.

Likewise, if component $C$ does not provide all the functionality required by $B$, one can compute a new provides-interface $\operatorname{prov}_{B}^{\prime}$ with $p_{B}$ :

$$
p_{B}^{-1}\left(\operatorname{req}_{B} \cap \operatorname{prov}_{C}\right)=: \operatorname{prov}_{B}^{\prime} \subseteq \operatorname{prov}_{B}
$$

Since $p_{B}$ is monotone, $p^{-1}$ is, too. With $\operatorname{req}_{B} \cap \operatorname{prov}_{C} \subseteq \operatorname{req}_{B}$ we have $\operatorname{prov}_{B}^{\prime} \subseteq \operatorname{prov}_{B}:=p^{-1}\left(\operatorname{req}_{B}\right)$. In this case we use a requires-parametric contract.

Technically, the parametric contract is specified by the service effect specification. The actual way what to specify to calculate the parametric contract depends on the interface model used. In case of protocol modelling interfaces, the service effect specification can be given by FSMs [20]. In case of quality of service modelling interfaces, only requires parametric contracts are used. This is because a provides parametric contract evaluates not to a concrete interface with QoS requirements, but to constraints which describe a set of possible requires interfaces. Anyhow, for QoS modelling interfaces the parametric contract is given by service effect specifications, as described in section 3.1.4. 


\subsection{Context}

\subsubsection{Motivation}

One of the most important arguments for component-based software development is the black-box reuse of components. Components are developed by third party vendors, who sell their products to multiple clients. Therefore, component developers cannot make assumptions on the underlying operating system and hardware as well as the usage profile and connected components. In other words, the context the component will be used in is unknown to component developers. Szyperski defines a software component as "a unit of composition with contractually specified interfaces and explicit context dependencies only. A software component can be deployed independently and is subject to a composition by third parties" [6].

This definition emphasises the importance of context dependencies and their explicit definition. However, it remains vague what is actually part of the context beyond the relationships defined by the provided and required interfaces of a component. One of those undefined dependencies is the underlying hardware that influences QoS attributes of a component, like performance and reliability. Especially for QoS predictions, knowledge about such dependencies to the context is needed in addition to functional specifications, like behavioural protocols [21] and service effect specifications [22].

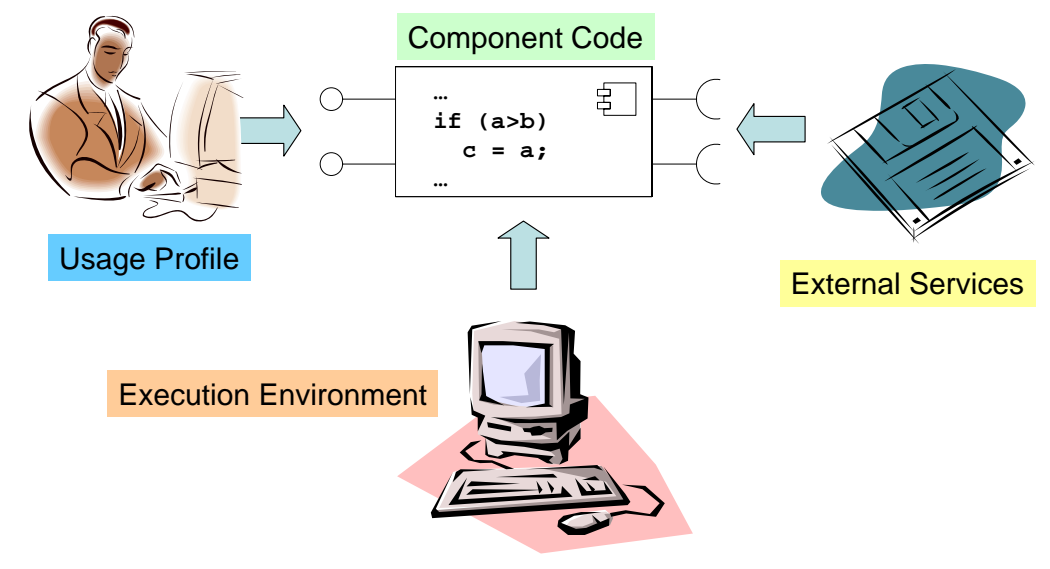

Figure 2.10: Influences on QoS properties of a software component.

Factors influencing the QoS attributes of a component can be classified into four main categories as shown in figure 2.10:

1. The implementation of the component, e.g. the selection of an algorithm.

2. The quality of required services, e.g. calling a slow or a fast service will result in a different performance for the provided service perceived by a user.

3. The runtime environment the component is deployed in. This includes the hardware and system software like the operating system and middleware platforms.

4. The usage of the component, e.g. if the component has to serve many requests per time span it is more likely to slow down.

With these four categories of influences we can define the quality of a provided service $s$ of a concrete component as a function of the varying influences. The implementation of the component's service is 
considered as a constant as it does not depend on its context but is fixed by the component developer at implementation time. Thus, the QoS of a component can be defined as a function of the remaining parameters, which are determined during its allocation, assembly and usage:

$$
q_{i m p l}: \mathcal{P}(s) \times D R \times U P \rightarrow Q
$$

where $\mathcal{P}(s)$ is the domain of the set of external services used by service $s, D R$ specifies the deployment relationship defining which component and connector is deployed on which part of the execution environment and $U P$ describes the usage profile. As a result, the function yields a value in the domain of the investigated quality metric $Q$.

\subsubsection{Context Influences}

Since QoS attributes of a component are strongly influenced by the environment the component is used in, the actual delivered QoS can only be determined knowing all influencing factors. We identified three aspects defined during system design that determine the complete context of a component based on the influences shown in figure 2.10: composition (connected components), usage, and allocation. For understandability, we split the influence of composition into the parts hierarchy and system/assembly and leave out the influence of the usage profile. All aspects are associated to different roles in the componentbased development process as described in section 2.1.

The structure of a system/assembly is defined by software architects who decide which components are used and how they are connected. Similarly component developers may construct composite components, which define the hierarchy of the system. Deployers define the execution environment and allocate software components among different resources, like servers and desktop computers.

System/Assembly (Horizontal Composition) A system specifies which components are used within an application and how they communicate. Within the system, the required interfaces of components are connected to provided interfaces of other components. That way it is determined which concrete external services are called by a component.

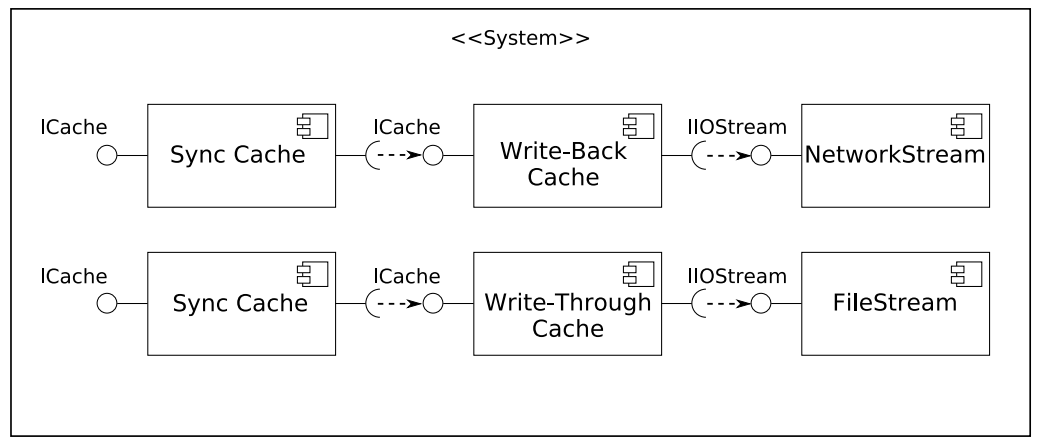

Figure 2.11: Component assembly.

A component can be used multiple times within a single system. Figure 2.11 illustrates this with a simple example. Three different types of components exist in the system shown there. On the right hand side, we have two I/O components that manage the access either to a file or network connection. Two different kinds of caching components that implement different caching strategies are shown in the 
middle. The SyncCache component on the left-hand side allows multiple tasks to access the caches concurrently without producing an incorrect state of the connected single-threaded caches.

The same component (SyncCache) is inserted at two different locations within the system. Both representations of the component are connected differently. Thus, users or other components that call the services provided by the different component representations will experience different QoS on the provided interfaces of the respective component representations. This is caused by the different caching strategies and I/O devices used by the SyncCache components. Modelling the component context explicitly allows us to hold the information on the diverse connections and the resulting quality attributes without changing the component specification.

Hierarchy (Vertical Composition) Related to the system, another important part of the context is the hierarchy in which a component is used. In figure 2.12, a composite component (BillingManager) is depicted which has been designed to create bills and store each one in a single PDF (Portable Document Format) file. The component is additionally supposed to write a summary of all the created bills as PDF file. Hence, the component PDFCreator is used in two different places. Notice however, that this kind of usage is usually unknown to the creators of the outer composite component. For them, the inner component (BillCreator) is a black box. They do not know the internal details and, hence, the usage of the inner PDFCreator is hidden.

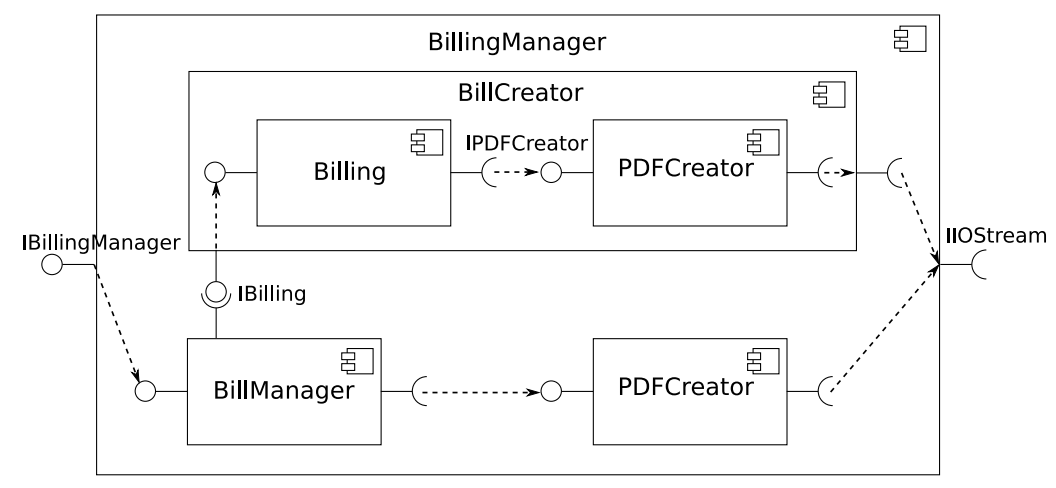

Figure 2.12: Component hierarchy.

In this case, the PDFCreator component is used in different contexts on different hierarchy levels. Note, that this only makes sense if the underlying component model supports hierarchical components at all. Considering parametric contracts, both components might offer different characteristics (QoS, functions offered, etc.). Additionally, they are used differently in their contexts. The PDFCreator of the inner component produces bills with less pages than the summary PDF file created by the outer PDFCreator.

Allocation An explicit context model is especially advantageous to model the allocation of components on hardware and software resources. Figure 2.13 depicts a system that uses replicated components to fulfil requests. In our example, server I is assumed to be slow and server II is assumed to be fast. Hence, the workload is not distributed equally, but $30 \%$ of the requests are directed to server I and $70 \%$ are directed to server II.

Here, we see several context influences. We have two copies of the same component allocated on 


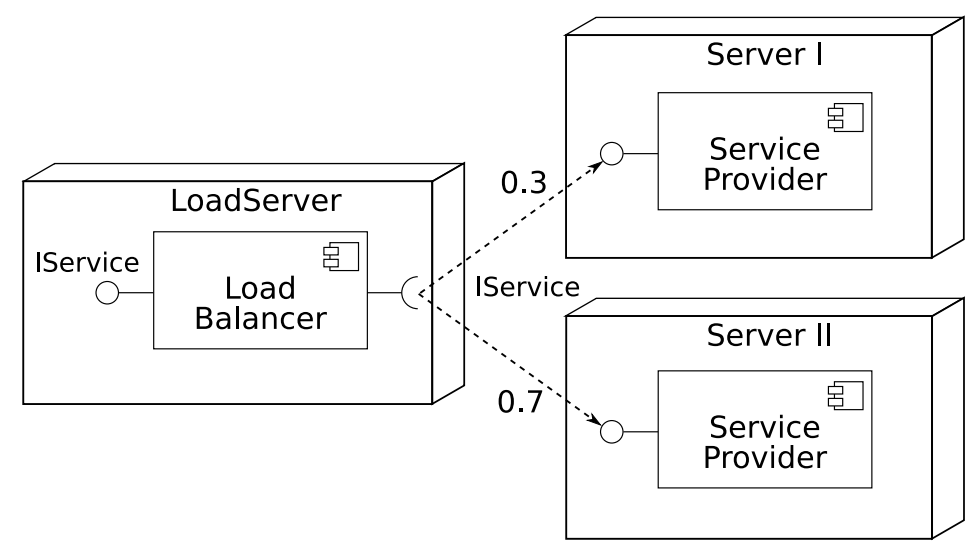

Figure 2.13: Component allocation.

different machines and, thus, in different contexts. The workload of each replicated component is different because of the distribution strategy. The processing power available to both replicated components is varying with the underlying hardware systems. However, both components are connected with an identical logical link going from the required interface of the workload balancer to the provided service of the replicated component. But again, each of these logical connections is most likely using a different physical communication channel, i.e., different network links.

\subsubsection{An Explicit Context Model}

In the previous section, we identified different input factors of the provided QoS of the same component in various contexts. In order to cope with these factors, we model a component's context explicitly as described in sections 3.2.3, and 3.3.5. Table 2.1 summarizes the attributes of our context model.

\begin{tabular}{|c|l|l|l|}
\hline & Software Architect & Deployer & Domain Expert \\
\hline \multirow{5}{*}{ Specified } & $\begin{array}{c}\text { System/Assembly } \\
\text { (see section 3.2.5) } \\
\text { Containment }\end{array}$ & $\begin{array}{l}\text { Deployed-on relation } \\
\text { (see section 3.3.5) } \\
\text { Execution environment } \\
\text { (see section 3.3.4) } \\
\text { - Component configuration } \\
\text { - Container properties }\end{array}$ & $\begin{array}{l}\text { System usage: } \\
\text { - Call probability } \\
\text { (see section 3.4.2) } \\
\text { - Call parameter } \\
\text { (see section 3.4.3) } \\
\text { - Workload }\end{array}$ \\
\hline Computed & $\begin{array}{c}\text { Results of parametric } \\
\text { contracts } \\
\text { (Functional) }\end{array}$ & \multicolumn{2}{|c|}{$\begin{array}{c}\text { QoS-Attributes } \\
\text { (Extra-Functional) }\end{array}$} \\
\hline
\end{tabular}

Table 2.1: Properties of the context

We arrange the table according to two dimensions. The vertical dimension distinguishes specified and computed attributes. Specified attributes are provided during the design process. They determine the computed attributes. The horizontal dimension divides the properties according to the roles of the 
development process that specify them: Software architects, deployers, and domain experts. While functional properties of the architecture can already be analysed on the basis of the system assembly, since only components and their connections are required to perform interoperability checks [21] or to evaluate parametric contracts [22], extra-functional attributes require additional information on the execution environment and usage of the system.

The deployer needs to specify the underlying hardware (CPU speed, cache sizes, available memory, available bandwidth, ...) and system software (details on the used middleware, virtual machines, container configurations, ...). The domain expert specifies probabilities for calling specific services, probability distributions on the actual parameter characteristics, or the request arrival rates. Using this information, it is possible to estimate QoS properties, like the actual execution time of a service on the specified runtime environment considering the specified usage profile. However, it depends on the capabilities of the analytical method, which information has to be specified and which QoS metrics can be derived. 


\subsection{Random Variables}

\subsubsection{Overview}

In the Palladio Component Model, we use random variables in expressions which specify parametric dependencies. The rationale behind this is that many aspects of larger software systems, especially in the business information systems area, can not be modelled having complete information (see for a classification of the information types for example [23]). Uncertainties can be found in many aspects of the system model. Two main sources stem from the behaviour of users and time spans of method executions (because we do not consider real-time environments). User behaviour can only be specified in a stochastical manner. How long users think (aka think time) between requests to the system, what parameter values they use in their requests can often only be characterized using probabilities. The second source comes from the facts that the PCM is designed to support predictions on an architectural level. On such a level, real-time constraints are usually not available. The reasons are also manifold. First, the information is simply not available at early design stages. Second, environmental features as garbage collectors, middle-ware services, etc. make it hard to predict timing time consumptions with certainty.

In the following, the use of random variables in parametric dependencies is introduced. These types of specifications are used in several places in the PCM. They are used especially in the ResourceDemandingSEFF and the ResourcePackage to describe resource consumptions. A library in the implementation of the PCM supports the use of random variables in different types. See 2.5.5 for technical details on this library.

\subsubsection{Definition}

Mathematically, a random variable is defined as a measurable function from a probability space to some measurable space. More detailed, a random variable is a function

$$
X: \Omega \rightarrow \mathbb{R}
$$

with $\Omega=$ the set of observable events and $\mathbb{R}$ being the set associated to the measurable space. Observable events in the context of software models can be for example response times of a service call, the execution of a branch, the number of loop iterations, or abstractions of the parameters, like their actual size or type. Note, that often a random variable has a certain unit (like seconds or number of bytes, etc.). It is important for the user of prediction methods to keep the units in the calculations and in the output to increase the understandability of the results.

A random variable $X$ is usually characterised by stochastical means. Besides statistical characterisations, like mean or standard deviation, a more detailed description is the probability distribution. A probability distribution yields the probability of $X$ taking a certain value. It is often abbreviated by $P(X=t)$. For discrete random variables, it can be specified by a probability mass function (PMF). For continuous variables, a probability density function (PDF) is needed. However, for non-standard PDFs it is hard to find a closed form (a formula describing the PDF). Because of this and for reasons of computational complexity, we use discretisized PDFs in our model.

For the event spaces $\Omega$ we support include integer values $\mathbb{N}$, real values $\mathbb{R}$, boolean values and enumeration types (like "sorted" and "unsorted") for discrete variables and $\mathbb{R}$ for continuous variables. 


\subsubsection{PDF discretisation}

A probability density function (PDF) represents a probability distribution in terms of integrals. The probability of an interval $[a, b]$ for a pdf $f(x)$ is given by the integral

$$
\int_{a}^{b} f(x) d x
$$

for any two number $a$ and $b, a<b$. To fulfill this property, $f(x)$ has to be a non-negative Lebesqueintegrable function $\mathbb{R} \rightarrow \mathbb{R}$. The total integral of $f(x)$ has to be 1 .

To create a discrete representation of a PDF, we use the fact that the probability of an interval is given by its integral. Basically, there are two ways to approximate a PDF, which mainly differ in the way how the intervals are determined. The first one uses a sampling rate and, therefore, a fixed interval size. The second one uses arbitrary sizes for intervals. Both methods store the probabilities for the intervals, not the probability density.

\subsubsection{Sampling - Fixed intervals}

To create an approximation of a PDF by a set of fixed intervals, the domain of the PDF is devided into $N$ intervals denoted by the set $I$, each of which has the same width specified by the value $d$. The $i$ th interval is then defined by $[(i-1 / 2) d,(i+1 / 2) d[$. For our purposes, we can assume that the domain of a PDF is always greater or equal to zero. Thus, we set the first interval $(i=0)$ to $[0,1 / 2 d[$. To minimize computational errors, we associate the probability of the $i$ th interval $[(i-1 / 2) d,(i+1 / 2) d[$ to its middle value $i * d$. So, we get a set of $N$ probabilities, where the probability of interval $i, p_{i}$ is given by the integral:

$$
p_{i}=\int_{(i-1 / 2) d}^{b} f(x) d x, \quad \lim _{b \rightarrow(i+1 / 2) d}
$$

for $i>0$ and

$$
p_{i}=\int_{0}^{b} f(x) d x, \quad \lim _{b \rightarrow 1 / 2 d}
$$

for $i=0$. The approximation of a PDF is completely described by the interval width $d$ and the probabilities $p_{i}$ for all intervals $I$.

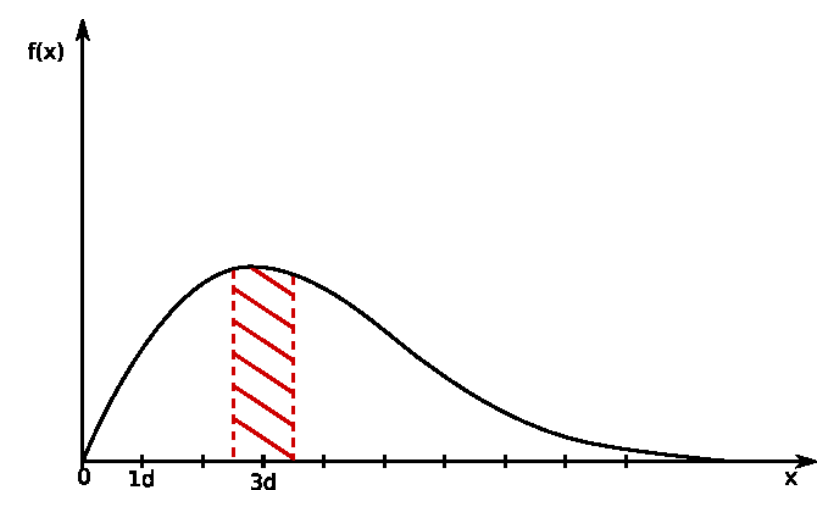

Figure 2.14: The probability of the interval $[2.5 d, 3.5 d[$ is the striped area under the graph. 
Example 2.1. Figure 2.14 illustrates how a pdf $f(x)$ is approximated by deviding its domain into a set of intervals. The $\mathrm{X}$-axis shows the multiples of the interval width $d(1 d, 2 d, 3 d \ldots)$. These values represent the mean values of the intervals to which the probabilities will be associated. The figure shows how the probability of the third interval is computed. The interval borders are given by $[2.5 d, 3.5 d[$ and its mean value is $3 d$. The probability $p_{3}$ is the striped area under the graph. So, all values lying in this area are associated to the value $3 d$.

\subsubsection{Approximation by boxes - Variable intervals}

For many PDFs, variable interval sizes allow a better approximation using less values compared to fixed ones. This is especially useful if the function consists of large, almost constant parts and sharp peaks on the other hand. Variable interval sizes allow the specification of almost constant areas by one large interval and the use of multiple, fine grained intervals for sharp peaks, which need to be described in more detail.

We have a set of intervals $I$ so that for each two intervals $J_{1}, J_{2} \in I, J_{1} \neq J_{2}$ the disjunction is the empty set $J_{1} \cap J_{2}=\emptyset$ and the union of all intervals forms a new interval from zero to $x \in \mathbb{R}^{+}$, $\cup_{J \in I}=[0, x[$. Intuitively, this means that the intervals do not overlap and that there are no gaps between the intervals.

To ensure both properties mentioned above, the intervals are specified by their right hand value only. Thus, we have a set $I_{X}$ whose values define the right hand sides of all intervalls. Suppose we can define an order on the set such that $x_{1}<x_{2}<\ldots<x_{n-1}<x_{n}$. Then the $i$ th interval is $\left[x_{i-1}, x_{i}[\right.$ for $i>1$ and $\left[0, x_{1}\right.$ [ for $i=1$. This allows us to specify $n$ intervals by $n$ values only and to ensure that the intervals neither do overlap nor have gaps inbetween. Now the probability $p_{i}$ for the $i$ th interval is given by

$$
p_{i}=\int_{x_{i-1}}^{b} f(x) d x, \quad \lim _{b \rightarrow x_{i}}
$$

for $i>1$ and

$$
p_{i}=\int_{0}^{b} f(x) d x, \quad \lim _{b \rightarrow x_{1}}
$$

for $i=1$.

\subsubsection{Functional random variables}

\subsubsection{General}

Additionally, it is often necessary to build new random variables using other random variables and mathematical expressions. For example, to denote that the response time is 5 times slower, we would like to simply multiply a random variable for a response time by 5 and assign the result to a new random variable. For this reason, our specification language supports some basic mathematical operations $(*,-,+, /, \ldots)$ as well as some logical operations for boolean type expressions $(==,>,<$, and, or,$\ldots)$.

To give an example, the distribution of a random variable $N$ is depicted in figure 2.15. The variable could model some characterisation of the size of a parameter of a component service.

To determine the time consumption of the method body which depends on the characterisation $N$ it is known that the amount of CPU instructions needed to execute the method is three times $N$. The resulting distribution function is shown in figure 2.16. 


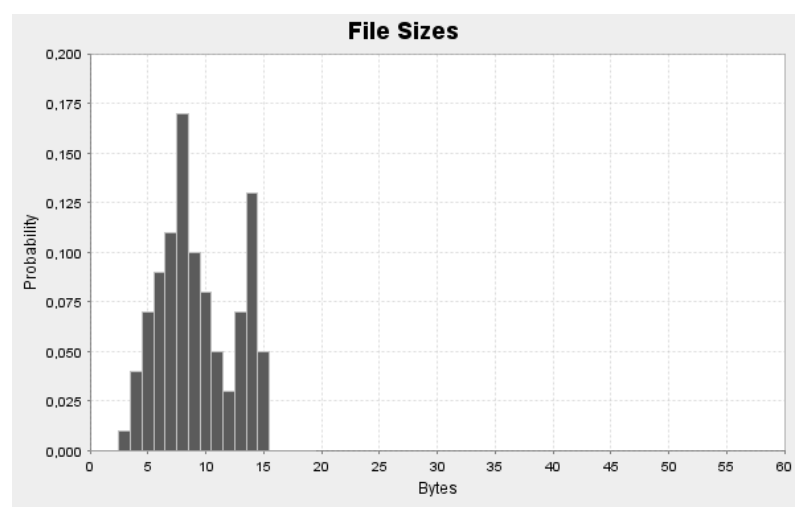

Figure 2.15: A Distribution of a Discrete Random Variable N

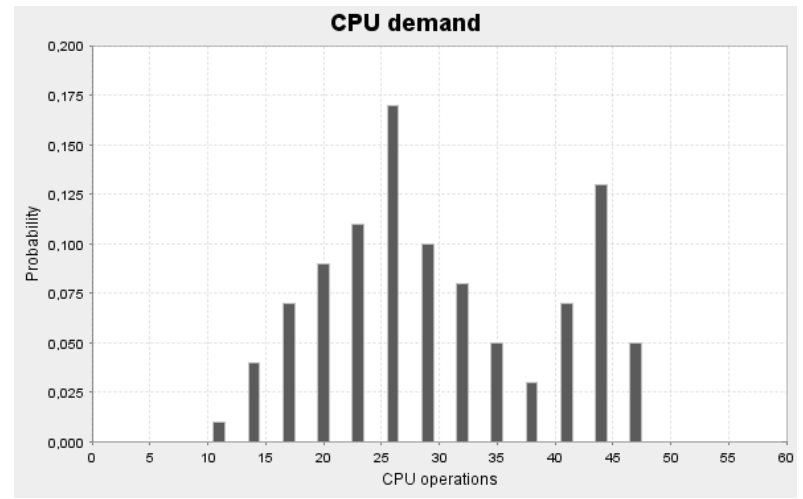

Figure 2.16: A Distribution of $\mathrm{N} * 3$

\subsubsection{Differences btw. discrete and continuous variables}

As introduced above we support the use of discrete as well as continuous variables. However, in such a case special care has to be taken when constructing expressions. Three times of a discrete variable can not be determined in the same way as three times a continuous variable. The reason for this is that continuous variables are also scaled continuously. To give an example, consider the continuous variable $\mathrm{X}$ which is uniformly distributed in a range between 5 and 10 seconds. If the variable is now multiplied by three, possible values of the resulting random variable can be in the interval between 15 and 30 seconds. The resulting distribution is again uniformly distributed having a density function which is one-third of the original density function.

An analogous example for a discrete random variable follows. Consider a discrete random variable taking the value 5 in $30 \%$ of all cases, 7 in $20 \%$ of all cases and 10 in the remaining $50 \%$. If this variable is multiplied by 3 , the result is a variable taking the value 15 in $30 \%, 21$ in $20 \%$ and 30 in $50 \%$ of all cases. The probabilities of the single events stay the same only the actual outcome changes.

The depicted difference is especially important in the case of discretisized PDFs. Any mathematical operation in which such a variable is involved has to treat the discretisized PDF as a 'real' PDF in order to avoid calculation mistakes. 


\subsubsection{Stochastic Expressions}

We call the language in which functional random variables can be specified Stochastic Expressions. As said before, the specifications of this kind of expressions is based on mathematical operations like addition or multiplication. The complete grammar is given below.

\subsubsection{Parser (EBNF)}

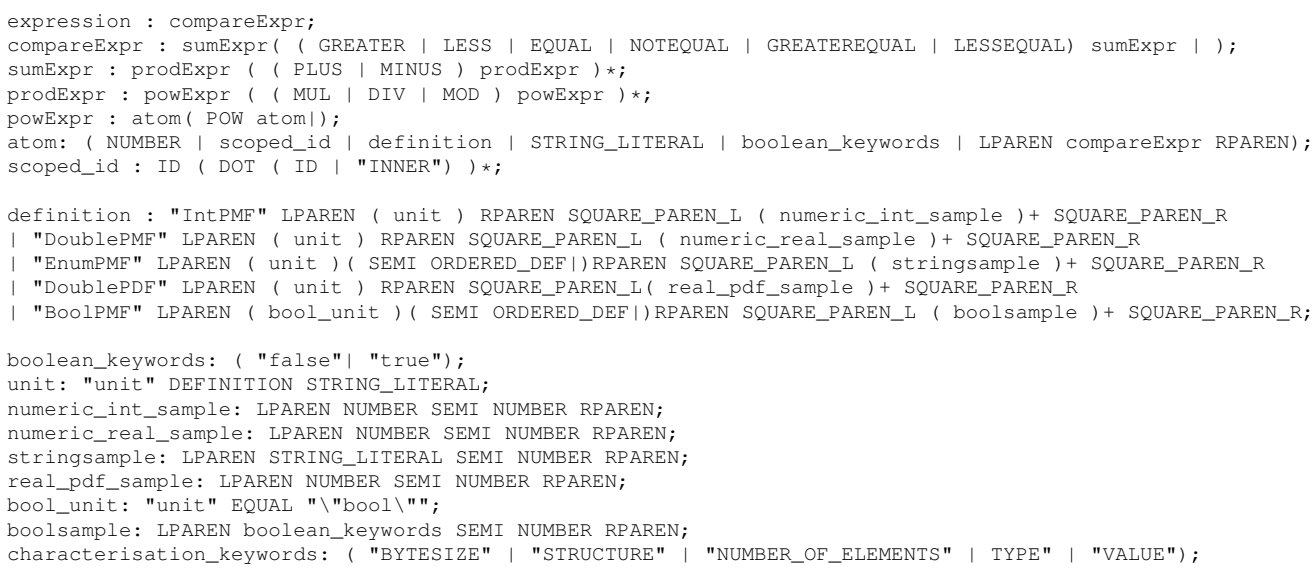

\subsubsection{Lexer (EBNF)}

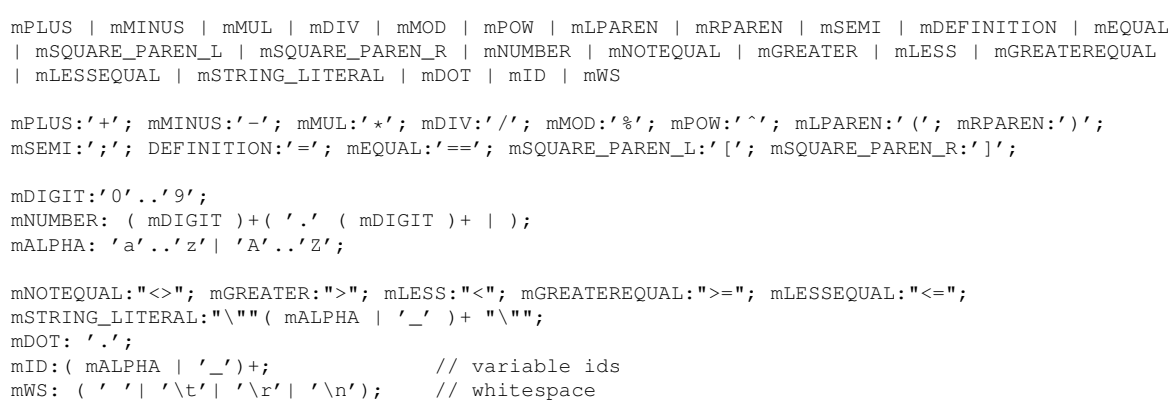

\subsubsection{Examples}

DoublePDF (unit="s") $[(1.0 ; 0.3)(1.5 ; 0.2)(2.0 ; 0.5)]$

- Specifies a time interval as boxed probability density function

- the unit is seconds

- the probability of the time being between 0 and 1 second is 30 percent $(0.3)$

- the probability of the time being between 1 and 1.5 seconds is 20 percent $(0.2)$

- the probability of the time being between 1.5 and 2 seconds is 50 percent $(0.5)$

- the probabilty of the time being longer than 2 seconds is 0 percent.

- all probabilities sum up to 1.0 
IntPMF (unit="iterations") [(27;0.1)(28;0.2) $(29 ; 0.6)(30 ; 0.1)]$

- Specifies the number of executing a loop as a probability mass function (PMF)

- the unit is iterations

- the probability of executing the loop exactly 27 times is 10 percent $(0.1)$

DoublePMF (unit=" ") $[(22.3 ; 0.4)(24.8 ; 0.6)]$

- Specifies a floating point variable charcterisation as a probability mass function (PMF)

- unit is omitted

- the probability of the variable taking the value 22.3 is 40 percent $(0.4)$

EnumPMF (unit="graphics") [ ("circle";0.2) ("rectangle";0.3) ("triangle";0.5) ]

- Specifies a probability mass function over the domain of a parameter

- Graphics-Objects can either be circles, rectangles, or triangles with the respective probabilities BoolPMF (unit="bool") [ (false;0.3) (true;0.7) ]

- Specifies a probabilty mass function for a boolean guard on a branch transition

- The guard is false with a probability of 30 percent and true with a probablity of 70 percent.

23

- An integer constant

- Can be used for example for loop iteration numbers, variable characterisations or resource demands

42.5

- An floating point number constant

- Can be used for variable characterisations and resource demands (not for loop iterations)

"Hello World!"

- A string constant

number.VALUE

- Characterises the value of the variable "number"

- You can assign a constant or probabilty function to a characterisation

- For example, number.VALUE = 762.3 or number.VALUE = DoublePMF(unit="') $[(22.3 ; 0.4)(24.8 ; 0.6)]$ 
graphic.TYPE

- Characterises the type of the variable "graphic"

- For example: graphc.TYPE = "polygon"

file.BYTESIZE

- Characterises the size of variable "file" in bytes

array.NUMBER_OF_ELEMENTS

- Characterises the number of elements in the collection variable "array"

- For example:

array.NUMBER_OF_ELEMENTS $=$ IntPMF (unit="elements") $[(15 ; 0.1)(16 ; 0.9)]$

set.STRUCTURE

- Characterises the structure of the collection variable "set"

- For example: sorted, unsorted

$2+4,34.3-1,88.2 * 1.2,14 / 2,60 \backslash \div 12$

number.VALUE * 15, file.BYTESIZE / 2

- Arithmetric expressions can combine constants

- Allowed are + (addition), - (substraction), * (multiplication), / (division),

- Arithmetric expressions may include variable characterisations

DoublePDF (unit="s") $[(1.0 ; 0.3)(1.5 ; 0.2)(2.0 ; 0.5)] \star 15$

IntPMF (unit="iterations") [(1124.0;0.3)(1125.5;0.7)] + 2.5

DoublePDF (unit="s") [(12.0;0.9) $(15 ; 0.1)]$ -

DoublePDF (unit="s") [ $(128.0 ; 0.3)(256 ; 0.2)(512.0 ; 0.5)]$

- Arithmetric expressions can also combine probability functions

number.VALUE < 20, fOO.NUMBER_OF_ELEMENT $==12$,

blah.VALUE $>=108.3$ AND fasel.TYPE == "mytype"

- Boolean expressions evalute to true or false

- You can use them on guarded branch transitions

- Valid operators are $>$ (greater), $<$ (less), == (equal), != (not equal), $\geq$ (greater equal), $\leq$ (less equal), AND, OR 
Chapter 3

Concepts 


\subsection{Component Developer}

\subsubsection{Overview}

Component developers are responsible for the implementation of software components. They take functional and extra-functional requirements for components to be developed and turn them into component specifications and executable software components. They may also receive specifications from other parties and implement components against them.

Component developers deposit their specifications and implementations into repositories, where they can be accessed by software architects to compose systems or by other component developers to create composite components. To provide an overview of the following section, Figure 3.1 contains an exemplary component repository, which contains most of the entities supported by the PCM.

Interfaces, components, and data types are first-class-entities in PCM repositories. They may exist own their own and do not depend on other entities. For example, Figure 3.1 contains the interface MyInterface (upper left), which is not bound to a component. The interface contains a list of service signatures. Interfaces may also contains protocol specifications, which restrict the order of calling its services, or QoS specifications, which describe their extra-functional properties. Section 3.1.2 describes interfaces in detail.

Components may provide or require interfaces. The binding between a component and an interface is called "provided role" or "required role" in the PCM. We distinguish provided and requires roles depending on the meaning of an interface for a component. For example, figure 3.1 contains the component A (top), which is bound to the interface YourInterface in a providing role. Section 3.1.3 details on the relationship between components and interfaces.

Common data types are needed in repositories, so that the signatures of service specifications refer to standardised types. In the PCM, data types can be primitive types, collection types, or composite types to build complex data structure. Figure 3.1 contains a PrimitiveDataType "INT" and a CollectionDataType "INT-Array", which only contains "INTs" as inner elements. Section 3.1.2 contains more information on data types.

Different types of components can be modelled in the PCM to a) reflect different development stages and b) to differentiate between basic (atomic) components and composite components.

Concerning a), Figure 3.1 contains the components B, which has a ProvidedComponent Type and does not contain required interfaces, $\mathrm{C}$, which has a CompleteComponent Type and contains no inner structure, and component $\mathrm{D}$, which has an ImplementationComponent Type and may contain an inner structure. Components may be refined from ProvidedComponentTypes to Implementationcomponent Types during design.

Concerning b), component E in Figure 3.1 is a composite component. The PCM is a hierarchical component model, which allows composing new components from other components. From the outside, composite components look like basic components, as they publish provided and required interfaces. Within the composite component, they use delegation connectors to forward requests to inner components and assembly connectors to bind inner components. Composite components may also include other composite components (notice component $\mathrm{G}$ within component $\mathrm{E}$ ).

For each provided service, basic components may include a mapping to required services, a so-called ServiceEffectSpecification (SEFF). It models the order in which required services are called by the provided service and may also include resource demands for computations of the service, which are needed for performance predictions. SEFFs are an abstract behavioural description of a component designed to preserve the black-box principle. Section 3.1.4 explains different types of SEFFs and their application. 


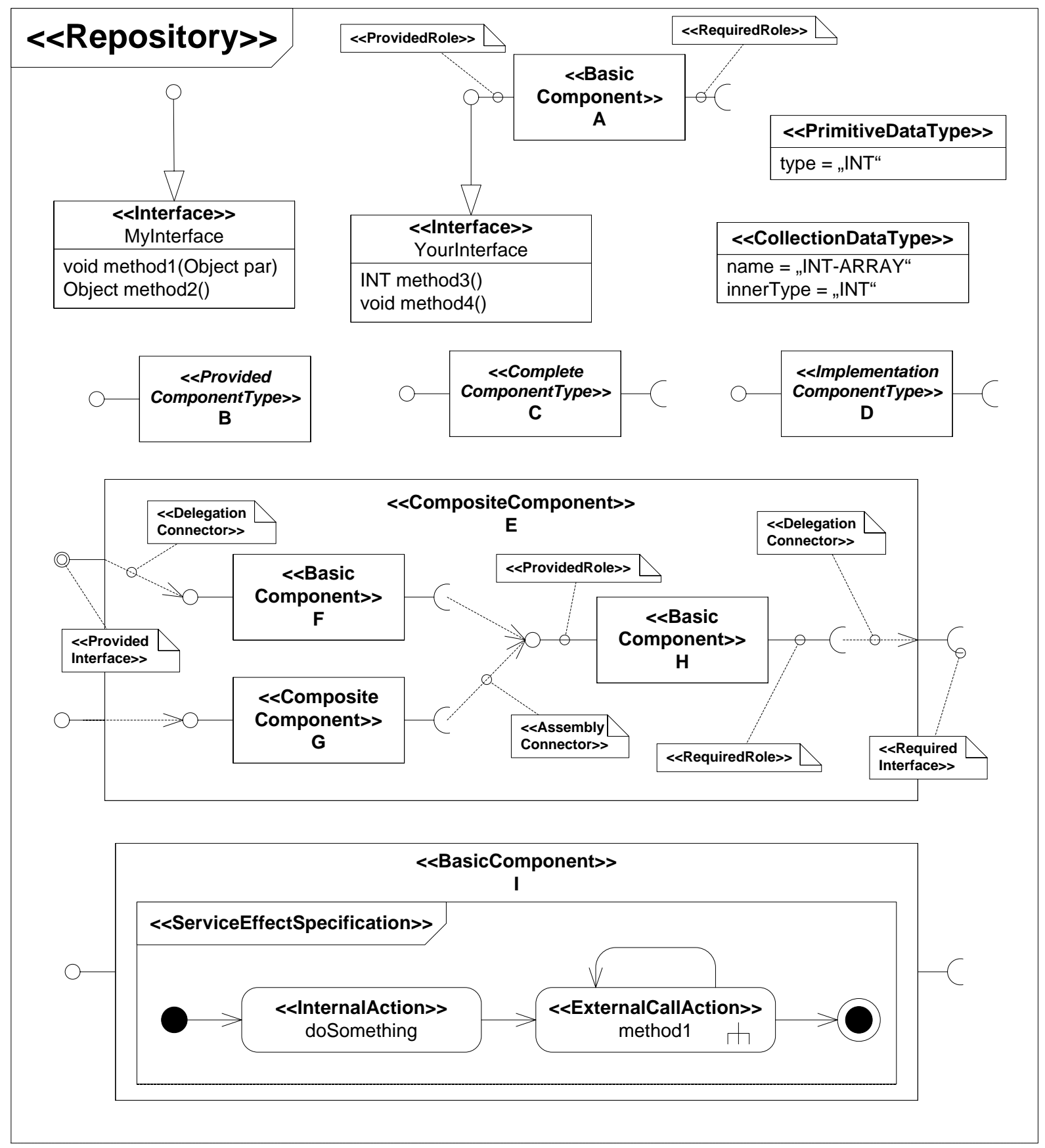

Figure 3.1: Repository Example 


\subsubsection{Interfaces}

Szyperski et al. emphasise the relation between components and interfaces: "Interfaces are the means by which components connect [6, p. 50]." For components, interfaces are a key concept serving multiple purposes. First, this section will describe the structure of PCM interfaces and then discuss the role of interfaces as contracts as well as inheritance of interfaces.

An interface within the PCM consists of a list of sigatures (mandatory), a protocol specification (optional). The following explains both concepts in more detail.

\subsubsection{Signatures}

A signature in the PCM is comparable to a method signature in programming languages like C\# or Java. It is widely compatible with the OMG's IDL standard [24, p. 3-1 and following]. Each signature of an interface is unique and contains:

- A type of the return value or void (no return value)

- An identifier naming the service

- An ordered set of parameters $\left(0 .{ }^{*}\right)$. Each parameter is a tuple of a datatype and an identifier (which is unique across the parameters). Additionally, the modifiers in, out, and inout (with its OMG IDL semantics, cf. [24, Chapter 3]) can be used for parameters.

- An unordered set of exceptions.

A signature has to be unique for an interface through the tuple (identifier, parameters). An interface has a list of $1 .{ }^{*}$ signatures and a signature is assigned to exactly one interface. However, different interfaces can define equally named signatures, which are distinguished by their parameters. If, for example, void doIt () is defined for interface A and B, void doIt () is not identical in both interfaces.

\subsubsection{Protocols}

A protocol is a set of call sequences to the services of a single interface and can be optionally added to an interface specification. In general, a protocol defines the set of all possible call sequences of the interface's signatures. Depending on the role of the interface (cf. Section 3.1.2.5), protocols are interpreted differently. Protocols of provided interfaces specify the order in which services have to be called by clients. Protocols of required interfaces specify the set of all possible call sequences to required services. However, the specification of a protocol is independent of its interface's role.

Figure 3.2 shows an example of protocols visualised as finite state machine. Nodes represent states, while edges represent calls to services and are labelled with signatures. The figure on the left hand side shows the protocol for the interface IReaderWriter as a finite state machine. First, open (..) is called. Then, read (..) and write (..) can be called in an arbitrary sequence. Finally, close() terminates the protocol.

Besides finite state machines, different formalisms can be used to model protocols. For example, Petri nets or stochastic process algebras could model interface protocols. However, the choice of a formalism implies the possible analyses. For example, to check the interoperability of two components, language inclusion has to be checked. The language inclusion is undecidable for Petri nets in the general case, so protocols modelled with Petri nets cannot be checked for interoperability. 

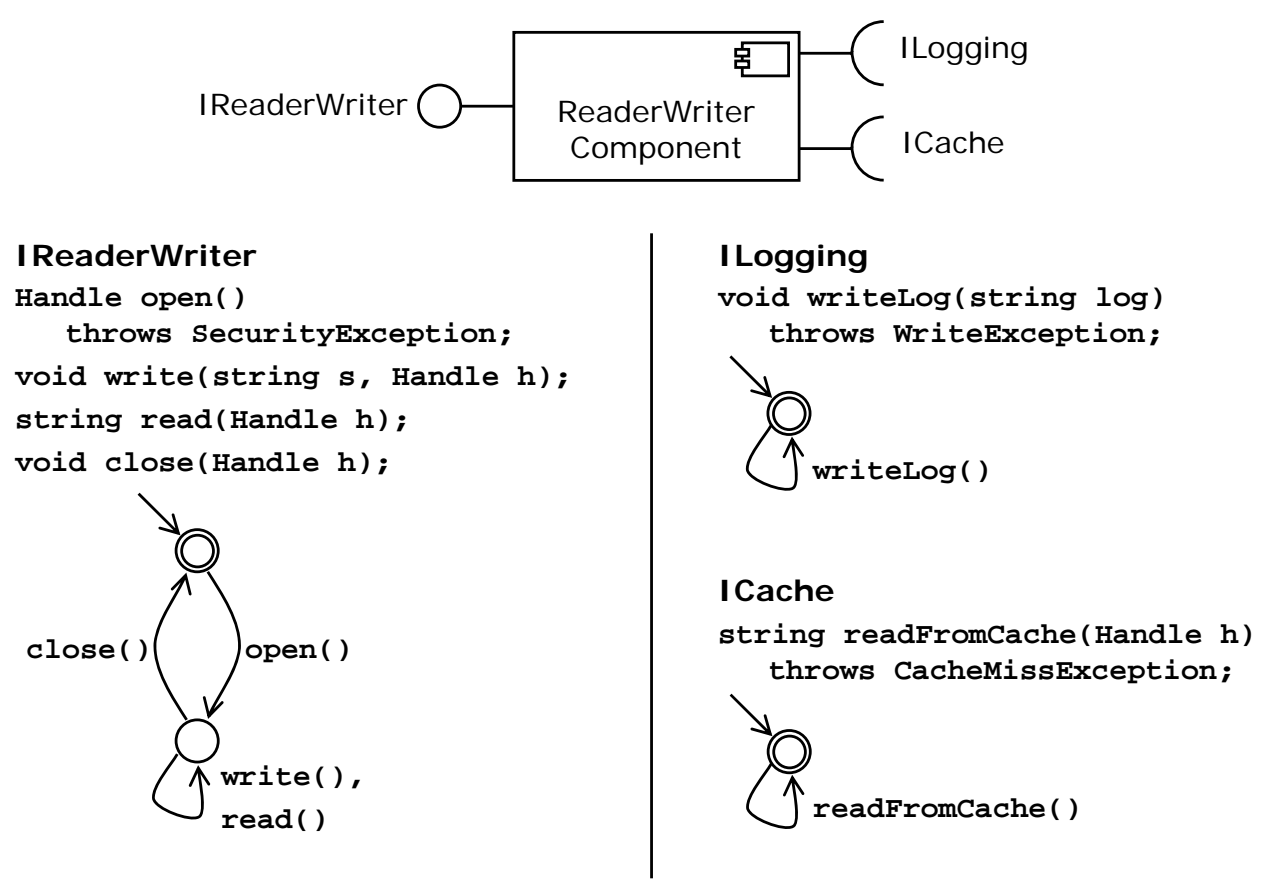

Figure 3.2: Example: Interfaces with Signature Lists and FSM protocols

\subsubsection{Interfaces as Contracts}

Interfaces are applied to specify the allowed communication between components. The contracts specified in the interface (method contracts, invariants) characterize the valid behaviour of these entities. In object-oriented languages an object can act in two roles with respect to an interface: server or client. In the server role, the object "implements" or "realizes" the operations specified in the interfaces and observes the method pre- and postconditions. In the client role, the object calls services offered in a given interface by fulfilling the precondition and expecting the postcondition. However, in both cases the interface and its associated contracts serve both roles as contract on which they can rely.

As with legal contracts, interfaces can exist even if no one actually declared their commitment to them, i.e., there is no specific client or server. For example, this is used to define a certain set of standardised interfaces of a library to enable the construction of clients and servers of these libraries independently. Thus, in our model the concept Interface exists as first class entity which can be specified independent from other entities.

An interface protocol is a special case of the more general concept of arbitrary preconditions for methods. Any kind of protocol can be expressed via preconditions. Thus, the protocol is an abstraction of the set of all preconditions. The abstraction is often based on the expressiveness of the used specification formalism.

\subsubsection{Interface Inheritance}

The subtype relationship of any two arbitrary interfaces $I_{1}, I_{2}$ can be specified as follows. Interface $I_{1}$ is subtype of $I_{2}$ if it is able to fulfil at least the contracts of $I_{2}$. In detail, this means it has to be able to handle all the (single) method calls which $I_{2}$ can handle. Additionally, it must also at least support the call sequences which $I_{2}$ supports. A common constraint for the hierarchy of interfaces is that any given 
interface can not be supertype of itself, which gives us a acyclic subtype hierarchy.

This definition of interface inheritance is required to support contra-variance - cases in which not the original interface is used, but a super- or sub-type. A sub-type can replace a super-type at the provided side of a component, while a super-type can be used instead of a sub-type at the required side.

\subsubsection{Roles}

Components use interfaces to declare their provided and required functionality. These interfaces are often referred to as provided and required interfaces. Since interfaces themselves can be considered as contracts that do not make any statement about the participants of the contract (cf. Section 3.1.2), the role of the component for the contract needs to be set elsewhere.

The PCM uses Roles for this purpose. A Role associates an interface to a component. The type of the association determines whether the component offers or demands the interfaces. ProvidedRoles reference the interfaces offered by a component. In this case the component takes the role of a server. It implements the services defined in the interfaces. Furthermore, it can rely on the call sequences defined in the interface's protocol, because its clients will adhere to it. On the other hand, RequiredRoles reference the interfaces requested by a component. The component uses the interfaces to implement its functionality. It will only call the its services according to the interface's protocol specification. 


\subsubsection{Components}

To create a software system, software architects can use existing components from repositories or specify new ones. So, some of the components in an architecture are already specified while others are only sketched. As a consequence, we cannot characterise component-based development processes into the classical top-down (i.e., going from requirements to implementation) or bottom-up (i.e., assembling existing component to create an application) categories. Instead, it is a mixture of both approaches.

This mixture needs to be reflected in the component model, since software architects must be able to use fully specified components in combination with nearly unspecified ones in their architectural descriptions. The PCM reflects this requirement by a so-called component type hierarchy. It distinguishes three abstraction levels for software component specifications (from abstract to concrete): provided types, complete types, and implementation types. On the most abstract level, provided types specify a component's provided interfaces leaving its requirements and implementation open. This allows software architects to create ideas of components, leaving their realisation unspecified. On the middle level, complete types fully specify a component's required and provided interfaces, but do not make any statements about its internal structure. This is more concrete than provided types as the component's dependencies have already been defined. However, the actual implementation (how provided services use required ones) still remains open. Software architects can use complete types for substitution of one component by another, if they have a selection of multiple components (e.g., different variants or versions) with the same functionality but different extra-functional properties. Last but not least, implementation types abstractly specify the internal behaviour of a software component. Their behavioural model describes how the provided services of the actual component implementation call its required services. Behavioural descriptions of software components are needed to evaluate extra-functional properties such as reliability or performance of software architectures.

This section provides an overview on the different component types and their relationships. It first introduces the ideas and concepts of the type hierarchy. Then, the realisation in the PCM and the meta model of the type hierarchy are explained. The following describes the concepts of the three levels of the type hierarchy. The explanation starts with the most concrete implementation type, since it conforms to the intuitive understanding of a software component for most developers. Based on the concepts introduced the more abstract concepts of complete and provided types are explained.

\subsubsection{Implementation Component Type}

Implementation (component) types include descriptions of a component's provided and required interfaces as well as abstract specifications of its internal structure. The specification of the internal structure depends on the way the component is realised. In general, components can either be implemented from scratch or composed out of other components. In the first case, the implemented behaviour of each provided service needs to be specified with a service effect specification (SEFF, cf. Section 3.1.4) to describe the component's abstract internal structure. We refer to such components as basic components, since they form the basic building blocks of a software architecture. On the other hand, developers can use existing components to assemble new, composite components. The internal structure of these components is the structure of the assembly (i.e., the included components and their interconnections). The following explains the concepts of basic and composite components in more detail

Basic Components Basic components are atomic building blocks of a software architecture. They cannot be further subdivided into smaller components. 


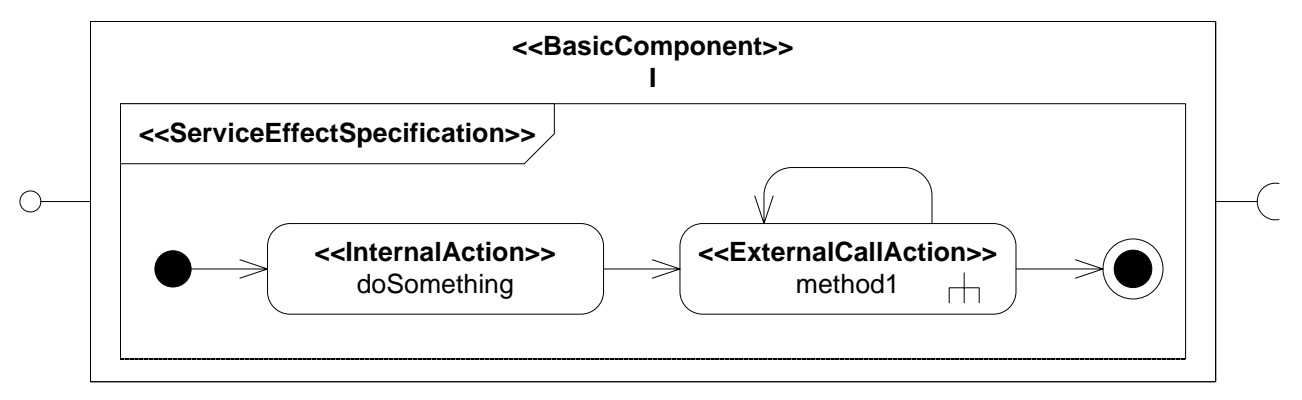

Figure 3.3: Example of a Basic Component.

Basic components encapsulate their internal structure (black box view). For reasoning about a basic component's properties, it may contain SEFFs, which describe the dependency between provided and required roles (cf. Figure 3.3). SEFFs abstract from the component's internal behaviour and only reveal necessary internals to reason on the component properties, such as protocol interoperability and QoS. Section 3.1.4 describes SEFFs in detail.

Composite Components Composite components are created by assembling other, existing components (cf. Figure 3.4). They base on composed structures (cf. Section 2.2.2), which contain a set of assembly contexts, delegation connectors, and assembly connectors. Assembly contexts embed components into the composite component. Assembly connectors bind required and provided roles of inner components in different contexts. Delegation connectors bind provided (required) roles of the composite component with provided (required) roles of its inner components.

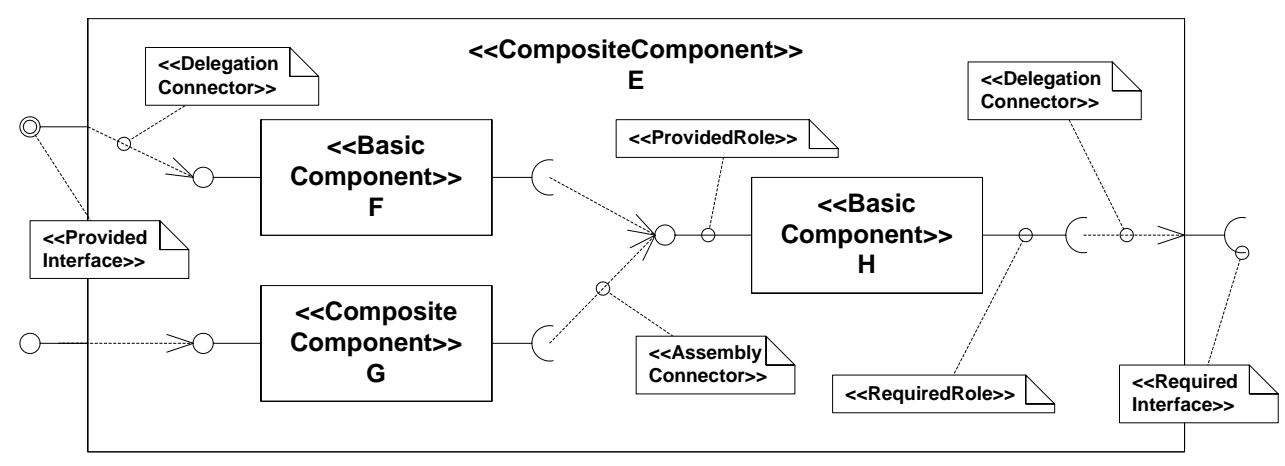

Figure 3.4: Example of a Composite Component.

Composite components may contain other composite components, which again are composed of other components. This enables building arbitrary hierarchies of nested components. Like basic components, composite components may contain SEFFs. However, these SEFFs are not specified manually by the component developer, but can be computed by combining the SEFFs of the inner components.

\subsubsection{Complete Component Type}

Complete (Component) types abstract from the realisation of components. They only have provided and required roles omitting the components' internal structure (i.e., the service effect specifications or encapsulated components). Thus, complete types represent a black box view on components. 
Software architects can integrate complete types into their architectures, which are fully connected with their provided and required roles. However, as their internal structures are not specified, they can be substituted by basic or composite components in later development stages. This is especially useful if the component is developed in a top down fashion. As described in Section 2.1.2, software architects can use complete types as a requirement specifications handed over to third parties. Component developers provide the actual implementations and specifications which complete the software architecture as soon as they are available.

If a component's implementation and specification does not exist, software architects can still model and evaluate an architecture. However, they have to provide basic QoS estimates for the complete component types in their architecture to evaluate its QoS attributes. Furthermore, the QoS results cannot be expected to be as accurate as for implementation component types.

\subsubsection{Provided Component Type}

Provided (Component) types abstract a component to its provided interfaces, leaving its requirements and implementation details open. So, provided types subsume components which offer the same functionality, but with different implementations and requirements. As different implementations might require different services from the environment, provided types omit required interfaces. Provided types allow software architects to focus on a component's functionality.

Using provided types, software architects can draft ideas on how functionality can be partitioned among different components without worrying about their implementation. In the initial phases of architectural design, it often does not make sense to arrange all details of a component, since most of them depend on the actual implementation and thus need to be specified by component developers. As during this phase the actual implementation is unknown, also the required interfaces of a component cannot be stated. However, software architects can still pre-evaluate software architectures containing providedtypes by giving basic QoS estimates for them. This gives rough estimates about the quality of a software system and defines QoS requirements for the component implementation.

\subsubsection{Type Hierarchy}

The provided, complete, and implementation component types can be organised in a hierarchy as shown in Figure 3.5. Provided types are on the top, most abstract level, since they only specify provided roles. The lower levels extend provided types with requirement and implementation specifications. On the middle level, complete types extend provided types with required roles, but still abstract from the actual implementation of a component. Implementation types on the lowest level of the hierarchy can either be composite or basic components. Thus, they specify the provided and required roles as well as the abstract internal structure of a component.

The different levels of the hierarchy are related to one another by the conforms and impl-conforms relationships. These relationships define under which conditions a component specification on a lower level is of a higher level type. A complete type conforms to a provided type if it offers at least the functionality specified in the provided type. Furthermore, an implementation type impl-conforms to a complete type if it offers at least the functionality of the complete type and requires at most the functionality of it required interfaces. The following explains both relationships in more detail and introduces a notion of substitutability based on their definition. 


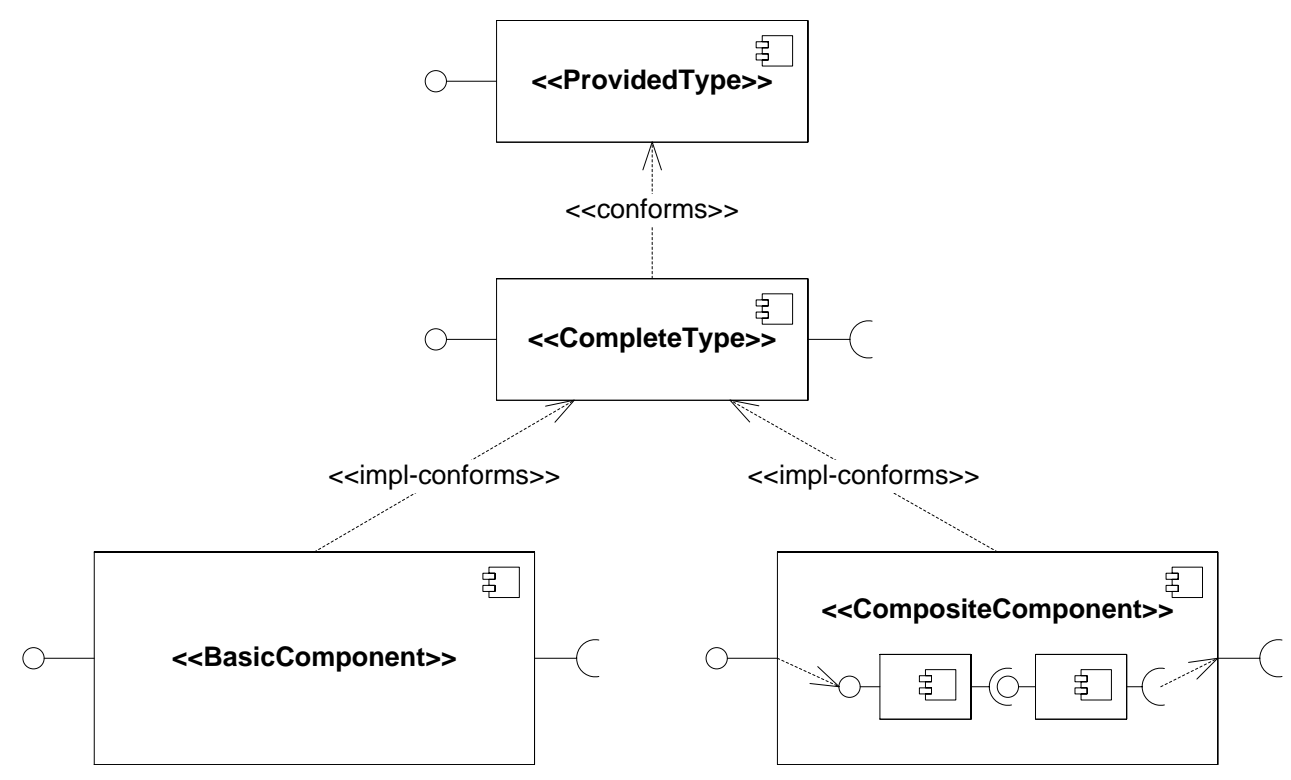

Figure 3.5: Component Type Hierarchy.

Conforms Relation The conforms relation is a subtype relation of provided and complete types. Abstractly speaking, a complete type conforms to provided type if it provides at least the functionality specified in the provided type. In order to concretise this statement, we need to define the provided functionality of a component and its relation.

Provided roles of components define their offered functionality associating interfaces to components. Thus, the conforms relation is defined on the provided interfaces of components ${ }^{1}$.

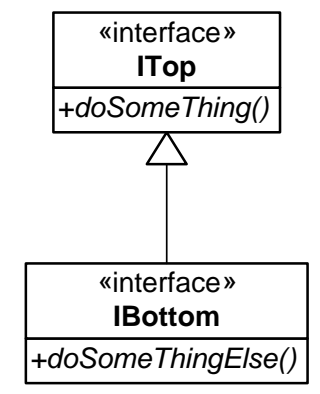

Figure 3.6: Example of interface inheritance.

Section 2.2 introduces the concepts of interfaces in the PCM. At this point, we only give a brief overview on the fundamental concepts. Interfaces are organised in an inheritance hierarchy. So, an interface can have multiple supertypes and subtypes. Basically, a supertype of an interface provides less and the subtype provides more services. Figure 3.6 shows an example. There, interface ITop is a supertype of interface IBottom while IBottom is a subtype of ITop.

\footnotetext{
${ }^{1}$ Another option would be the definition of the conforms relation on services provided by a component neglecting the corresponding interfaces. However, this is ambiguous, since two interfaces can provide syntactically equal services, but with different semantics. Furthermore, the PCM allows the specification of protocols for interfaces, which have to be considered in the conforms relation.
} 
In order to give a meaningful definition of the conforms relation, we have to consider the inheritance hierarchy of interfaces. We can refine the definition of the conforms relation.

A complete type conforms to a provided type if it provides at least the interfaces or subtypes of the interfaces specified in the provided type. More formally, let Prov be the set of provided interfaces of a component type including all supertypes. Then a complete type $\mathrm{C}$ conforms to a provides type $\mathrm{P}$ if $\operatorname{Prov}_{P} \subseteq \operatorname{Prov}_{C}$, the interfaces provided by $\mathrm{P}$ are a subset of the interfaces provided by C.

Implementation-Conforms Relation The impl-conforms relation is a subtype relation between implementation types and complete types. Abstractly speaking, an implementation type (either a basic or composite component) conforms to a complete type if it provides the same or more functionality and requires the same or less functionality than the complete type.

With respect to the provided functionality, the impl-conforms relation is similar to the conforms relation. In addition, the required functionality of an implementation type must be less or equal to the required functionality of a complete type. Analogously to the provided functionality, the required functionality is specified in the components' required roles (i.e., the interfaces associated to the component with its required roles). Considering the supertype and subtype relation of interfaces, we can define the impl-conforms relation as follows.

An implementation type conforms to a complete type if it provides at least the interfaces or subtypes of the interfaces provided by the complete type and if it requires at most the interfaces or supertypes of the interfaces required by the complete type. More formally, let Prov be the set of provided interfaces of a component type including all supertypes and $R e q$ be the set of required interfaces of a component type including all subtypes. Then an implementation type I conforms to a complete type $\mathrm{C}$ if $\operatorname{Prov}_{C} \subseteq$ Prov $_{I}$ and $R e q_{C} \supseteq R e q_{I}$, the interfaces provided by $\mathrm{C}$ are a subset of the interfaces provided by $\mathrm{I}$ and the interfaces required by $C$ are a superset of the interfaces required by $I$.

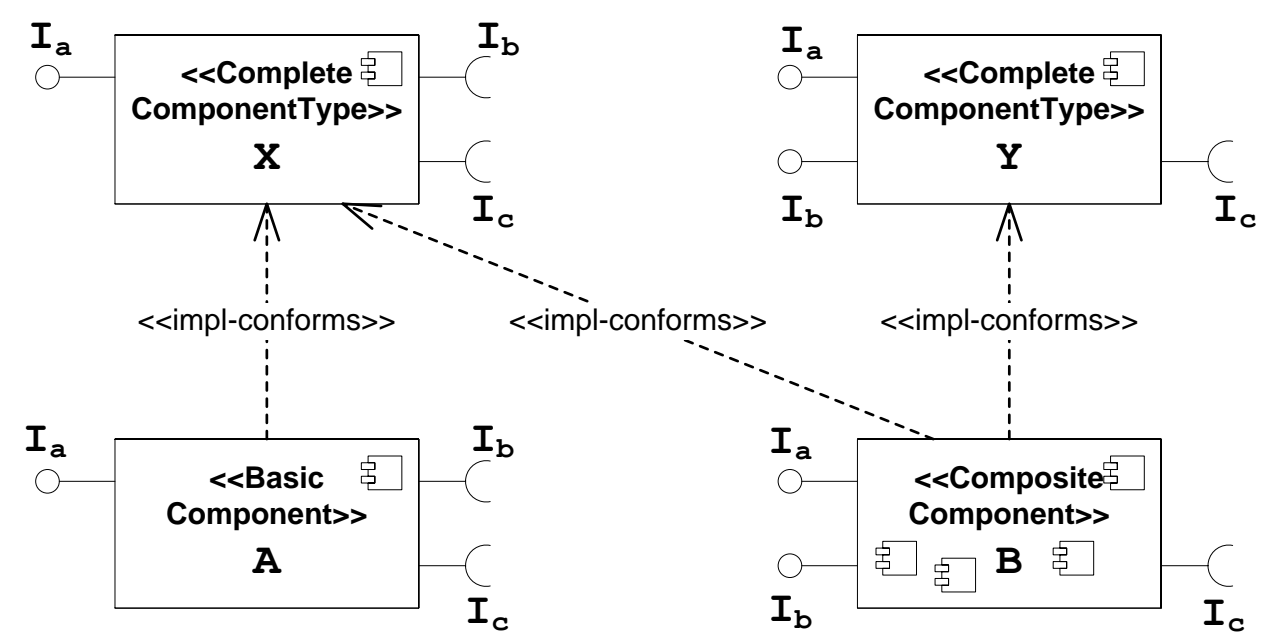

Figure 3.7: Example for Component Type Conformance.

Figure 3.7 shows an example for conforms relations of implementation and complete components. Complete component $X$ provides interface $I a$ and requires $I b$ and $I c$ while complete component $Y$ provides $I a$ and $I b$ and requires $I C$. Basic component $A$ provides and requires the same interfaces as complete type $\mathrm{X}$ and thus impl-conforms to $\mathrm{X}$ as indicated by the dashed arrow with the stereotype 
$<<$ impl-conforms >>. However, A does not conform to $Y$ since it does not provide interface $I b$ and additionally requires it. Composite component $\mathrm{B}$ impl-conforms to both types $\mathrm{X}$ and $\mathrm{Y}$ as it provides interfaces $I a$ and $I b$ and only requires $I C$.

Cardinality of the Conforms Relations The conforms as well as the impl-conforms relations are many-to-many relations between two levels of the component type hierarchy. Each implementation type can conform to multiple complete types and each complete type can be implemented multiple times. Figure 3.7 illustrates this. Composite component $\mathrm{B}$ impl-conforms to complete types $\mathrm{X}$ and $\mathrm{Y}$ and complete type $\mathrm{X}$ is implemented by basic component $\mathrm{A}$ and composite component $\mathrm{B}$. The same holds for the conforms relation as well. Each provided type can abstract multiple complete types and each complete type can conform to multiple provided types.

Substitutability The main application of both conforms relations is the definition of substitutability for software components in the PCM. A component can substitute another component if it conforms to its type. Depending on the type of conforms relation, the substitution of a software component can have different effects. The following discusses this in more detail.

Assume we have a software architecture where an implementation type $A$ is used. If a component $B$ shall substitute $A$ and $B$ conforms to the provided type of A, but not impl-conforms to its complete type, $B$ provides at least the interfaces offered by $A$, but requires additional interfaces. Thus, replacing $A$ by $B$ in the given architectures can lead to problems since not all of its requirements are fulfilled. On the other hand, a component $B^{\prime}$ that impl-conforms to $A$ can easily replace $A$, since it provides the necessary interfaces and all its requirements can be fulfilled by the surrounding architecture.

\subsubsection{Type Hierarchy Meta Model}

A part of the meta model describing the component type hierarchy is analogous to the structure of the type-hierarchy itself (cf. Figure 3.8). Each type level is a specialisation of the upper levels. So, lower levels in the hierarchy only add information to the component specification, e.g. the complete type adds mandatory required roles. Thus, lower type levels inherit the attributes of the upper levels.

However, the inheritance between the different type levels is only partially connected to the conforms relations. As a consequence of the inheritance, an instance of a basic component is as well an implementation, complete, and provides type. Due to the definition of the conforms relation, it certainly conforms to itself. However, the conforms relation is not restricted to itself, it can conform to other component types as well. 


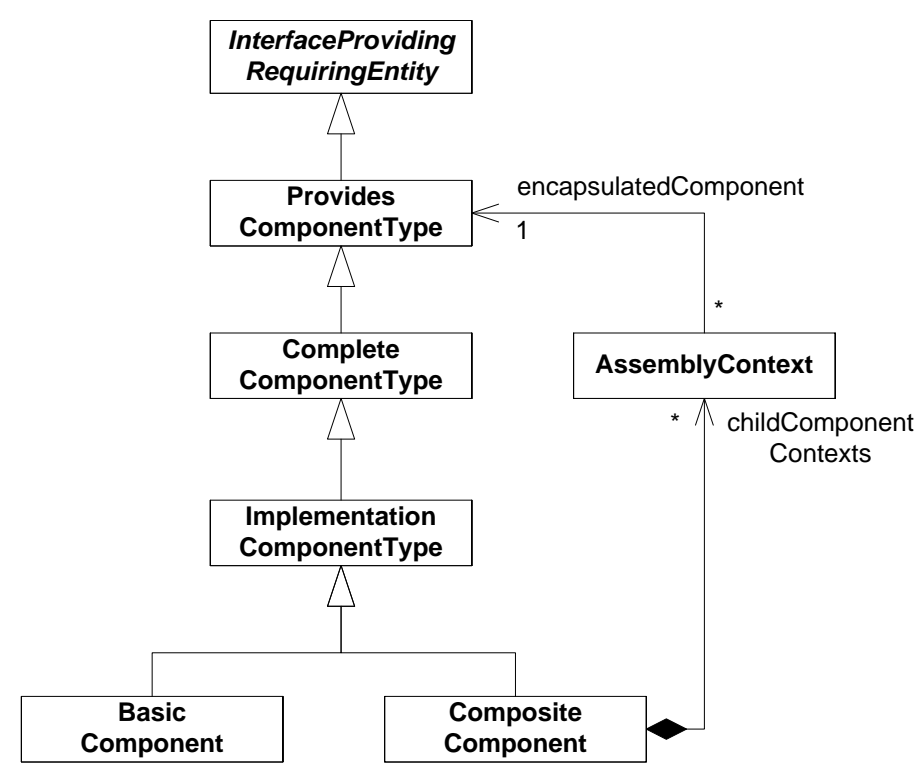

Figure 3.8: Meta Model of the Component Type Hierarchy.

\subsubsection{Service Effect Specification}

\subsubsection{Motivation}

The goal of the PCM is to provide modeling capabilities that enable QoS analyses of component-based software architectures. As clients perceive different QoS characteristics of a provided service in a component-based architecture depending on a particular context, component developers have to provide parameterised specifications of the QoS attributes of their components. Such context dependencies for a specific component service may originate from a) input parameters (including the current component internal state), b) resource usage, and c) usage of required services. These influences have to be made explicit in the service's specification.

To achieve accurate QoS analyses, a description of the usage of required services (influence c)) for each provided service of a component is useful, because the QoS characteristics perceived at the provided interface can depend on QoS characteristics of calls to required services. For example, consider a provided service calling a slow required service. In this case, the response time of the provided service will be perceived as slow by its clients, because the execution time of the slow required service has to be included in its own execution time (details can be found in [25]). software architects cannot know how requests to a provided service of a component are propagated to required services if no dependencies between them are specified. Thus, component developers have to enhance their component specifications with a description of such intra-component dependencies to enable accurate specification-based QoS analyses by third parties.

\subsubsection{Description}

A service effect specification (SEFF) describes how a provided service of a component calls its required services and is thus an abstraction of the control flow through the component. In the simplest case, a SEFF of a provided service is a list of signatures of the services in the component's required interfaces. For more sophisticated analyses, a SEFF can be modelled as a finite state machine (FSM), which captures 
sequences, branches, and loops. In any case, a SEFF captures the externally visible behaviour of a provided service while hiding its internal computations.

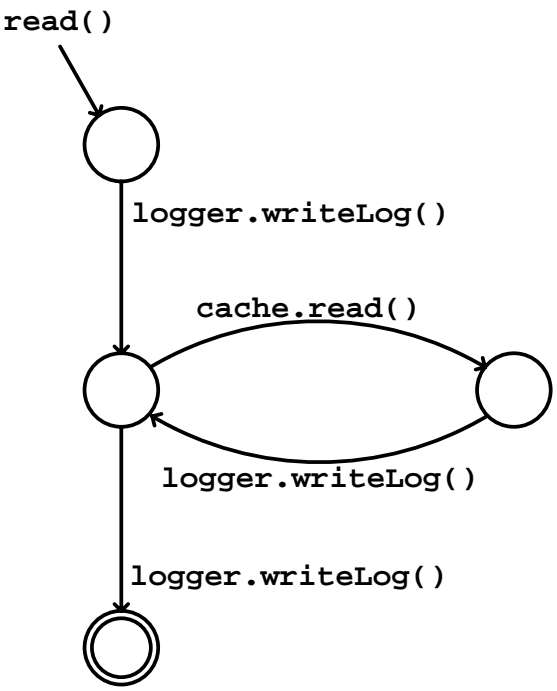

(a)

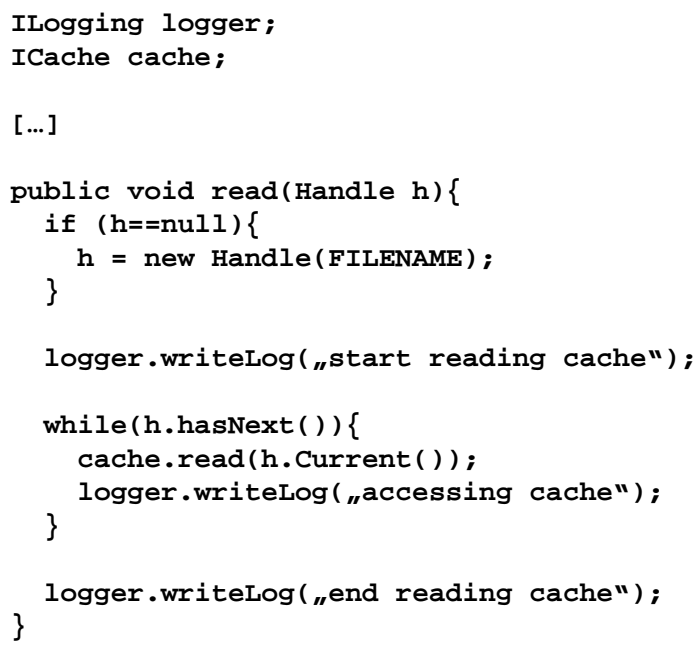

(b)

Figure 3.9: Example SEFF as FSM and corresponding source code

Example 3.1 (FSM-SEFF). In figure 3.9(a), a SEFF is modeled as a FSM for the provided service read, whose source code is shown in Figure 3.9(b)). This service first initialises a file handle, writes to a log file, and then reads from a cache within a loop. After completing the file access, another entry is added to the log. In the FSM, edges represent calls to required services and are annotated with the name of these services. The states abstractly represent the internal computations of a service after or before executing a required service. Notice, that the SEFF only contains the sequence of calls to the required services, while the component internal activity of initialising the file handle is abstracted.

Although SEFFs reveal the inner dependencies between provided and required interfaces of a component, they do not violate the black box principle. First, these specifications are only used by tools performing analyses, and do not have to be understood by humans. Second, they do not reveal the intellectual property of component developers encoded in the service's algorithms, because they are a strong abstraction of the component's source code. Third, in many cases, these specification can be generated out of byte code components, which are generally considered black box components.

SEFFs can be specified for basic components by the component developers and computed for composite components out of the SEFFs specified for the inner components [12]. For existing legacy basic components with available source code, the SEFFs have to be specified manually so far. However, in the future it is planned to implement analysis tools for component source code to assist component developers in the SEFF specification of legacy components by semi-automatically generating them.

\subsubsection{Types of Service Effect Specifications}

Different types of SEFFs besides simple service lists and FSMs can be modelled to support different kinds of analysis (e.g., protocol checking, QoS analysis, etc.). If different SEFF types are defined for the same provided service, a mapping should exist between the FSM SEFF and the other types of SEFFs, 
which ensures the same names for provided and required services and the same order of calls to required interfaces.

SEFFs have been introduced by Reussner [12], who has used them in the context of parameterised contracts for protocol adaptation (Section 2.3). In that work, counter-constraint automata are used to model SEFFs restricting the number of calls to specific required services. Furthermore, using Petri nets to model SEFFs is envisioned to support concurrent component behaviour. However, assuring protocol interoperability is not possible if the component behaviour is modeled with Petri nets, because the language inclusion problem is undecidable for them in the general case.

While plain FSMs are well-suited for restricted protocol checking, they are generally insufficient for QoS analyses, because additional stochastic information and QoS characteristics (such as execution times or reliability values) are needed. Thus, several other forms of SEFFs have been proposed. For reliability prediction, Reussner and Schmidt [20] enhance SEFF FSMs with transition probabilities, so that they become Markov models. Similar Markov models enhanced with distribution functions for execution times have been used for performance predictions by Firus et. al. [4]. Happe et. al. [26] propose modeling SEFFs as stochastic Petri nets to enable QoS analyses involving concurrency. Koziolek et. al. [27] use stochastic regular expressions as SEFFs to make component-based performance predictions. These expressions are similar to Markov models, but are hierarchically structured and contain special constructs to model loops. Koziolek et. al. [3] use annotated UML 2.0 activities as SEFF models in the context of performance analysis. In [1] so-called resource demanding SEFFs have been introduced for QoS analysis, which have become part of the PCM and are described in Section 3.1.4.4.

In the PCM, a basic component can contain any number of SEFFs for each provided service, but at most one SEFF of each type, such as FSM or Petri net. A restriction on a particular SEFF type is deliberately avoided to enable different kinds of analyses. At the point of writing, the only SEFF type explicitly included in the PCM is the resource demanding SEFF. However, other types can be included in the PCM by inheriting from the class ServiceEffectSpecification. Consistency between different SEFF types has to be ensured by component developers, as it is not checked by the component model. If component developers implement a component based on a SEFF, it has to be ensured that the language of the SEFF is a superset of the language of the implementation.

\subsubsection{Resource Demanding Service Effect Specification}

A resource demanding service effect specification (RDSEFF) [1] is a special type of SEFF designed for performance and reliability predictions. Besides dependencies between provided and required services of a component, it additionally includes notions of resource usage, data flow, and parametric dependencies for more accurate predictions. Its control flow is hierarchically structured and can be enhanced with transition probabilities on branches and numbers of iterations on loops. In the following, the meta model of the RDSEFF will be illustrated, and its design rationale will be explained. For understanding and clarity, the illustration of the meta model and the concept descriptions are spread over several paragraphs.

Overview Figure 3.10 shows how RDSEFFs are connected to the PCM and contains their main parts. Each BasicComponent can contain a number of ServiceEffectspecifications, each of which references a signature of a provided service of the component. Each provided service can be described with different types of SEFFs.

A ResourceDemandingSEFF is a ServiceEffectSpecification and a ResourceDemandingBehaviour at the same time inheriting from both classes. The reason for this construct lies in the fact, that ResourceDemandingBehaviours can be used recursively inside themselves 


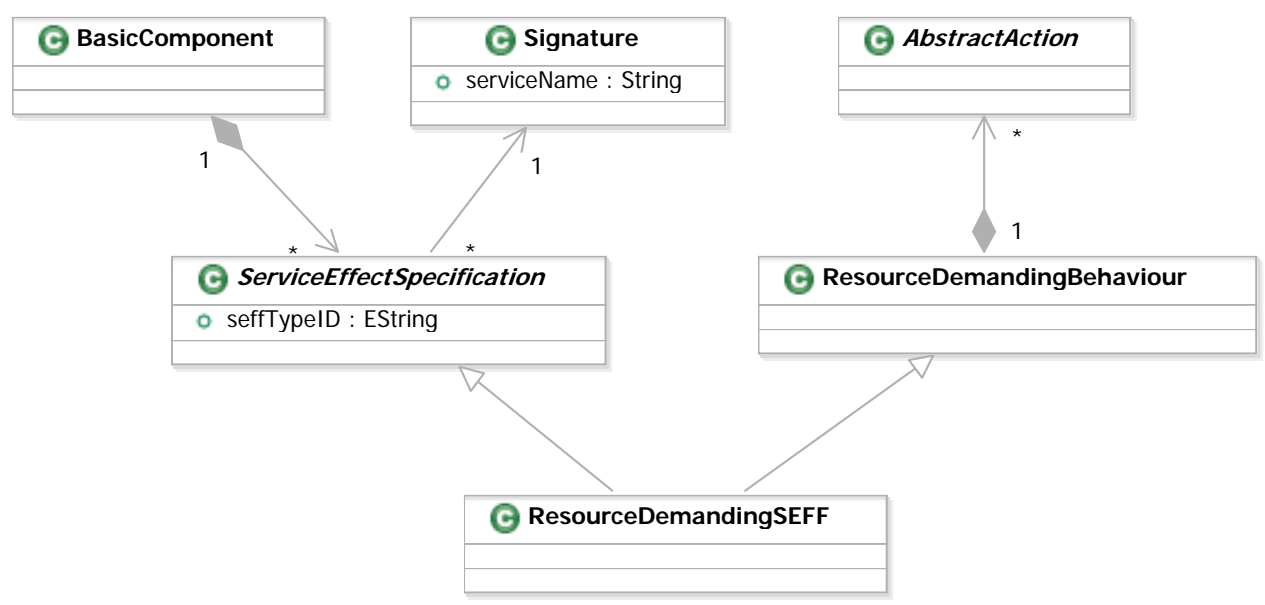

Figure 3.10: Overview of the RDSEFF

to describe loop bodies or branched behaviours (explained later), and these inner behaviours should not be RDSEFFs themselves.

The ResourceDemandingBehaviour is designed to reflect different influence factors on the performance and reliability of a component service. It contains a set of AbstractActions to model

- calls to required services,

- resource usage by internal activities, and the

- corresponding control flow between required service calls and resource usage.

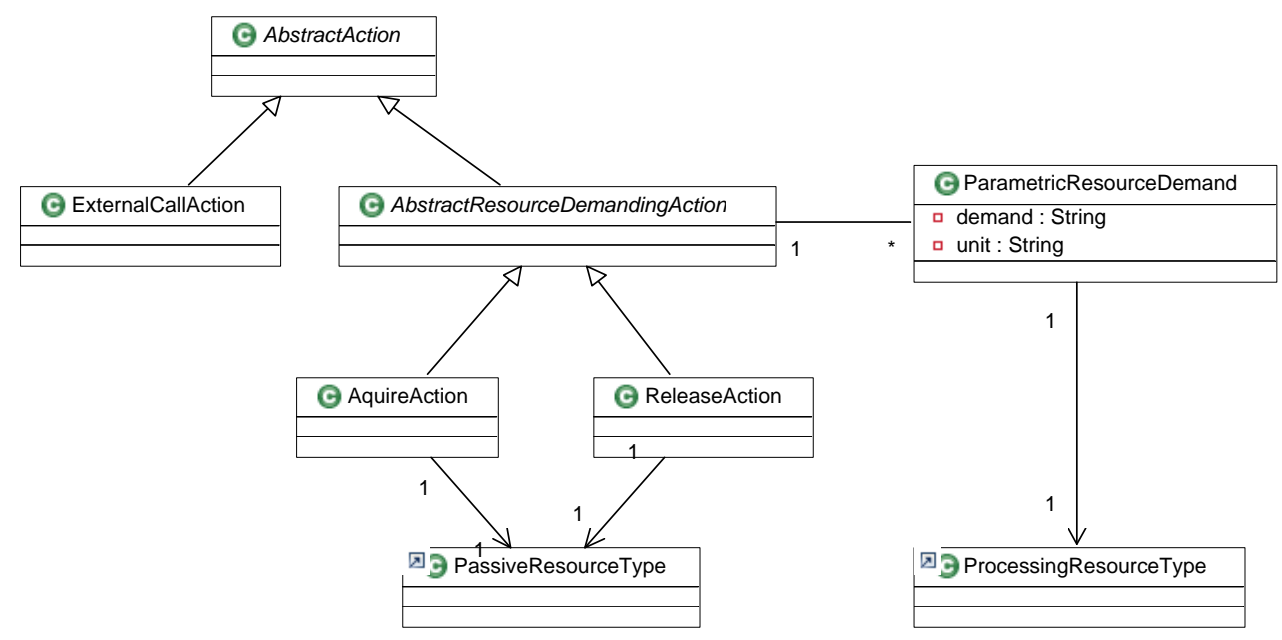

Figure 3.11: Resource Usage in RDSEFFs

Resource Demand To conduct QoS analyses, component specifications must contain information on how system resources, such as hardware devices or middleware entities are used by components. Ideally, component developers would specify a timing value for the execution time of each provided service of 
a component. However, these timing values would be useless for third party users of the component, because they would depend on the specific usage profile, hardware environment, software platform, and attached required services the component developer had used while measuring them.

Thus, component developers have to specify the demand each provided service places on resources instead of a timing value. Other than a timing value, the demand is independent from concrete resources. For example, a component developer could specify the number of CPU cycles of a specific operation within a service or the number of bytes read from or written to an I/O device. These demands have to be specified against abstract resource types, because the component developer does not know all possible resources the component could be deployed on. Only software architects and deployers know the concrete resources the component shall be used on and can define a specific deployment context (i.e., a resource environment model, Section 3.3.4). With this concrete context, for example, the execution time of one CPU cycle or the time to read one byte from an I/O device is specified. Then, actual timing values can be derived from the resource demands.

Resource demands of a component service may vary depending on how the service is used. For example, the hard disk demand of a component service, which offers downloading different files from a server, strongly depends on the size of the file that is requested via an input parameter. Another example would be the CPU demand of a component that allows sorting collections. Its CPU demand for the sort operation would depend on the number of elements in the collection. Thus, it could not be specified as a fixed value by the component developers, because they cannot forsee how the component will eventually be used by third parties. Therefore, it is necessary to specify resource demands in dependency of input parameters.

These considerations have been mapped to the meta model of the RDSEFF (see Figure 3.11). AbstractActions can either be external calls (ExternalcallAction), which reference required services and do not produce resource demands themselves, or internal computations actions (AbstractResourceDemandingActions), which actually place demands on resources. These ParametericResourceDemands contain a demand (e.g.,"127") and a unit (e.g., "bytes"). The demand can be specified in dependency to the service's input parameters (e.g., demand="x.BYTESIZE * 200", unit="CPU cycles", where " $x$ " is an input parameter of the service). Once "x.BYTESIZE" is specified by third party users, the actual resource demand can be computed.

Resource demands reference ProcessingResourceTypes from the ResourceType package of the PCM (Section 3.3.3). Once the concrete processing resource, such as a CPU or network device, is specified, the actual resource demands can be placed on them to calculate timing values.

Besides active resources, such as CPUs, I/O devices, storage devices, memory etc., component service may also acquire or release passive resources, such as semaphores, threads, monitors, etc. These resources usually exist in a limited number, and a service can only continue its execution if at least one of them is available. Passive resource are themselves not able to process requests and do not allow to place demands on them. They can only be acquired and released, which can be modelled with the AquireAction and ReleaseAction (see Figure 3.11). These actions reference PassiveResourceTypes from the resource type package of the PCM.

External Calls and Parameter Usage RDSEFFs allow the specification of calls to required services with ExternalCallActions, which are themselves AbstractActions (see Figure 3.12), but produce no resource demands directly. The resource demand produced by executing a required service has to be specified in the RDSEFF of that service. ExternalCallActions reference the signature of a required service.

Classical FSM SEFFs only specify the name of a called required service, but do not include the 


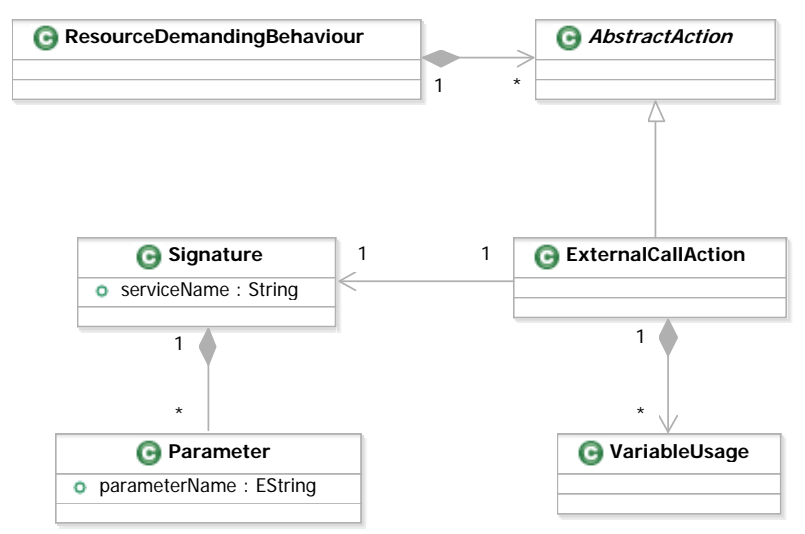

Figure 3.12: External Service Calls and Parameter Usage in RDSEFFs

parameter values of this call. They are control flow oriented and neglect the data flow. However, input parameters, which represent the concurrent data flow, can have a significant impact on the resource usage of a called service. Therefore, characterisations of parameter values passed to required services should be included into the RDSEFF by the component developers. They can either specify these characterisations if the component is still being designed. Or, after completing the component implementation, they can derive these characterisations from the source code.

It is possible that input parameters passed to a required service do not receive fixed or constant values within the calling component service. They might in turn depend on the input parameters of the calling service. These input parameters are however unknown to the component developers. Therefore, in such a case, the component developers have to specify a dependency (instead of a constant characterisation) between input parameters of the calling service and input parameter passed to required services.

In the PCM, VariableUsages can be used to specify the needed dependencies between parameters (Figure 3.12), which abstractly characterise the data flow through a component service. These variable usages are aligned to the parameter model described in Section 3.4.3. With them, it is possible to characterise the value, byte size, or type of primitive parameters as well as the number of elements or the structure of collections. The characterisations can be expressed as random variables (for details refer to Section 3.4.3).

Control Flow RDSEFFs extend classical FSM SEFFs with additional constructs for modeling control flow of the dependencies between provided and required interfaces (Figure 3.13). All control flow constructs are aligned in a hierarchical fashion that avoids ambiguities and eases analyses (an example will follow). A ResourceDemandingBehaviour contains a chain of AbstractActions, which each reference at most a single predecessor and successor. The first element of the chain is a StartAction, which has no predecessor, while the last element of the chain is a StopAction, which has no successor.

InternalActions should be used to reference ParametricResourceDemands for activities inside the described service, between calls to required services. In the future, they could be used to characterise the inner resource demand of basic components more detailed.

BranchActions split the control flow with an XOR-semantic: Exactly one of the attached $\mathrm{Ab}-$ stractBranchTransitions is taken when such an action is executed. Branches may result from if/ then/ else or case statement of the underlying source code. 


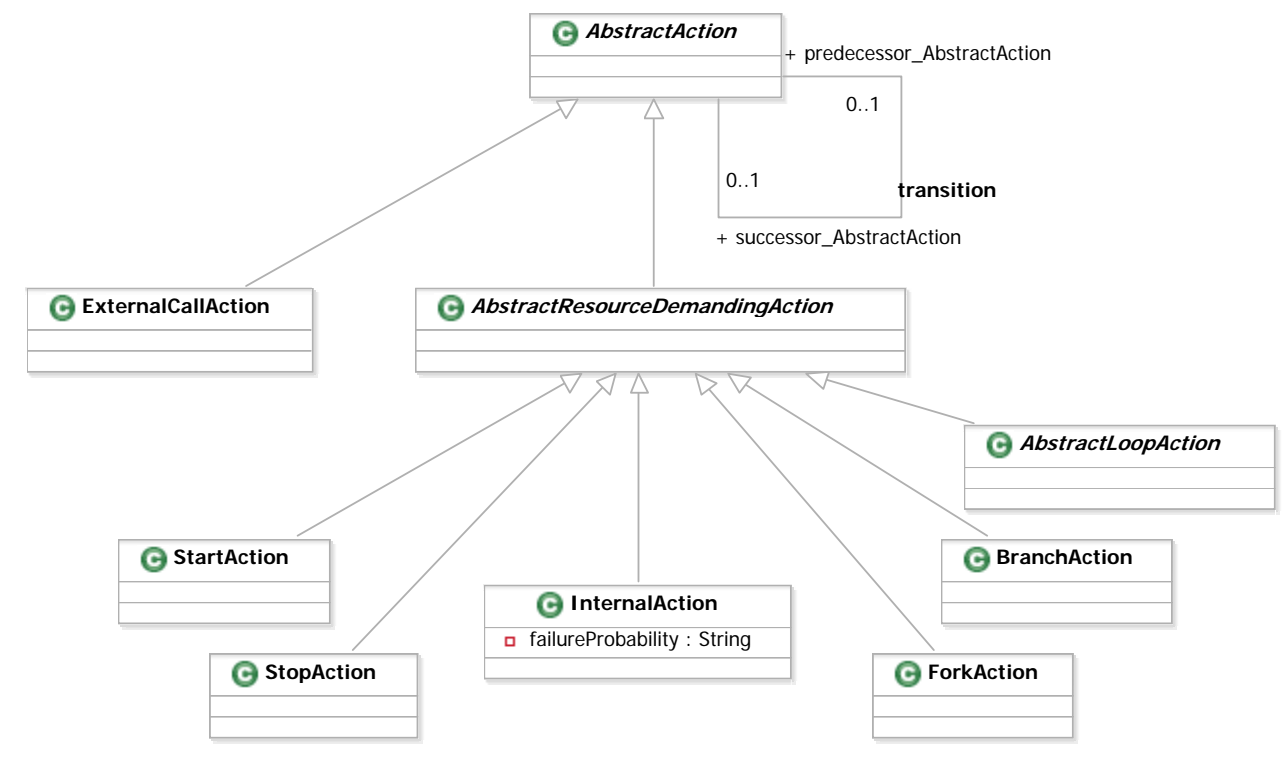

Figure 3.13: Control Flow in RDSEFFs

Branch transitions can be either guarded or probabilistic (Fig. 3.14). GuardedBranchTransitions, contain a branch condition as a random variable. For example, a branch condition could be connected to the value of an input parameter (" $x<1$ "), in which case a branching probability could be computed once the value of the input parameter is known. ProbabilisticBranchTransitions directly contain a probability and not a branch condition. They can be used in case a component developer cannot specify a guard related to input parameters or just to ease the analyses.

Additionally, each type of branch transition contains a ResourceDemandingBehaviour to model the inner actions of the branch. Using inner behaviours avoids the need to have a merge action to join branches. Furthermore, it prevents problems, which might arise when a nested "else"-branch cannot be associated unambigiously with an according "if"-branch.

ForkActions split the control flow with an AND-semantic: Each of the inner forked ResourceDemandingBehaviours has to be executed (possibly concurrently) before the control flow continues with the successor of the corresponding ForkAction. Forks may for example result from the invocations of threads. Because the inner activities of the forked behaviours are encapsulaed in ResourceDemandingBehaviours, there is no need for a join action to reconnect concurrent control flows.

Abstract LoopActions, like BranchTransitions and ForkActions, contain inner ResourceDemandingBehaviour, which include actions carried out in the loop body (Fig. 3.15). Loops can originate from for or while statements of the underlying source code.

Concrete loop action can be modelled either with LoopActions or CollectionIteratorActions. The former contain the number of iterations as a random variable, and this random variable can include dependencies on input parameters (explained later). The latter enables modeling the special but common case of iterating over a collection. Because of this, CollectionIteratorActions reference an input parameter of the current component service. This input parameter must be a collection parameter and the number of elements in this collection has to be characterised with a random variable. Then the loop gets executed for each element in the collection.

Notice, that for LoopActions, it is assumed that the parameters characterisations used in the loop body are stochastically independent, whereas for CollectionIteratorActions it is assumed that 


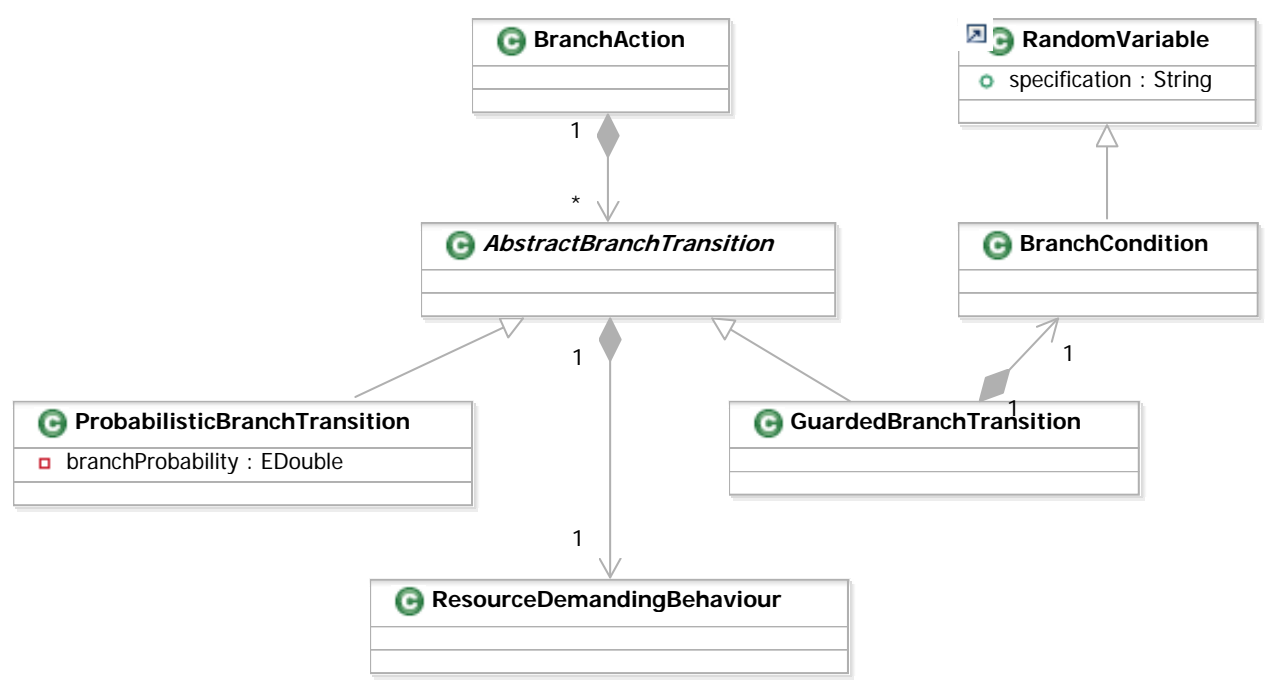

Figure 3.14: Branches in RDSEFFs

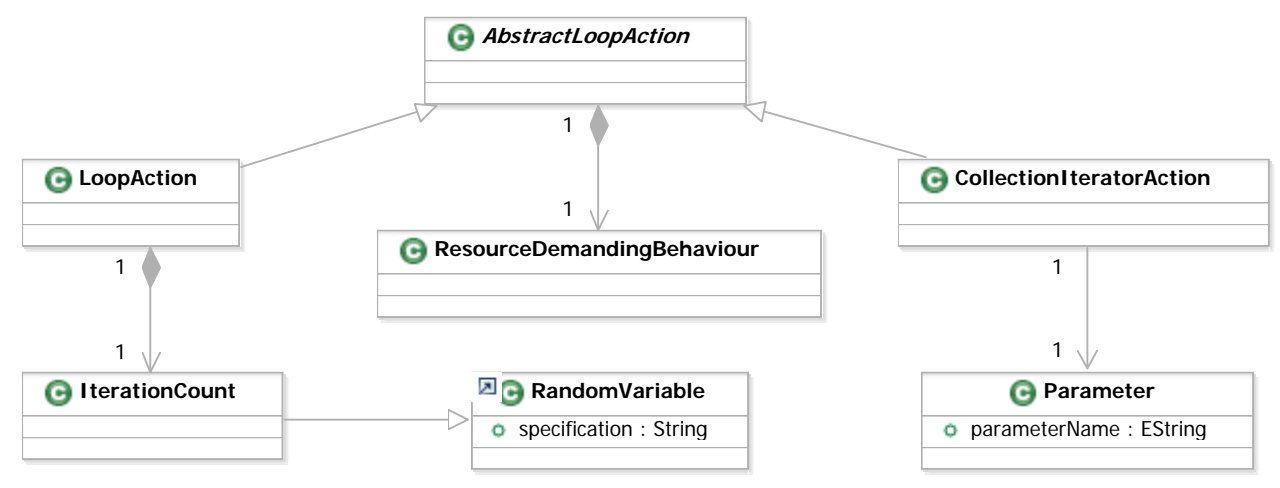

Figure 3.15: Loops in RDSEFFs

the characterisations are not independent. For example, if the characterisation of a parameter value is specified by a random variable and is used by two external call actions within a loop body, the analyses algorithms have to assure, that the second action uses the same characterisation as the first action and that the random variable does not get evaluated a second time.

Modelling loops with inner behaviours instead of allowing cyclic references in the chain of $\mathrm{Ab}-$ stractActions has several advantages [27]. In Markov models, loops are specified with cycles, so that there is an entrance probability for each loop and an exit probability. The probability of entering the loop decreases if the number of loop iterations is increased. For example, entering a loop with a entrance probability of 0.9 , leads to a probability of 0.81 for two loop iterations, and a probability of 0.729 for three loop iterations. Thus, the number of loop iterations is always limited to a geometrical distribution, which does not resemble practical situations well. Fixed number of loop iterations can only be specified by unrolling the loop to a number of states in Markov models. With the approach described above, it is possible to attach an arbitrary distribution function for the number of iterations to each loop.

Figure 3.16 shows a simplified example instance of an RDSEFF, which highlights the control flow concepts introduced before. Note that the constructs are hierarchically structured. Analysis algorithms can easily traverse the abstract syntax tree to make model transformations or QoS predictions. 


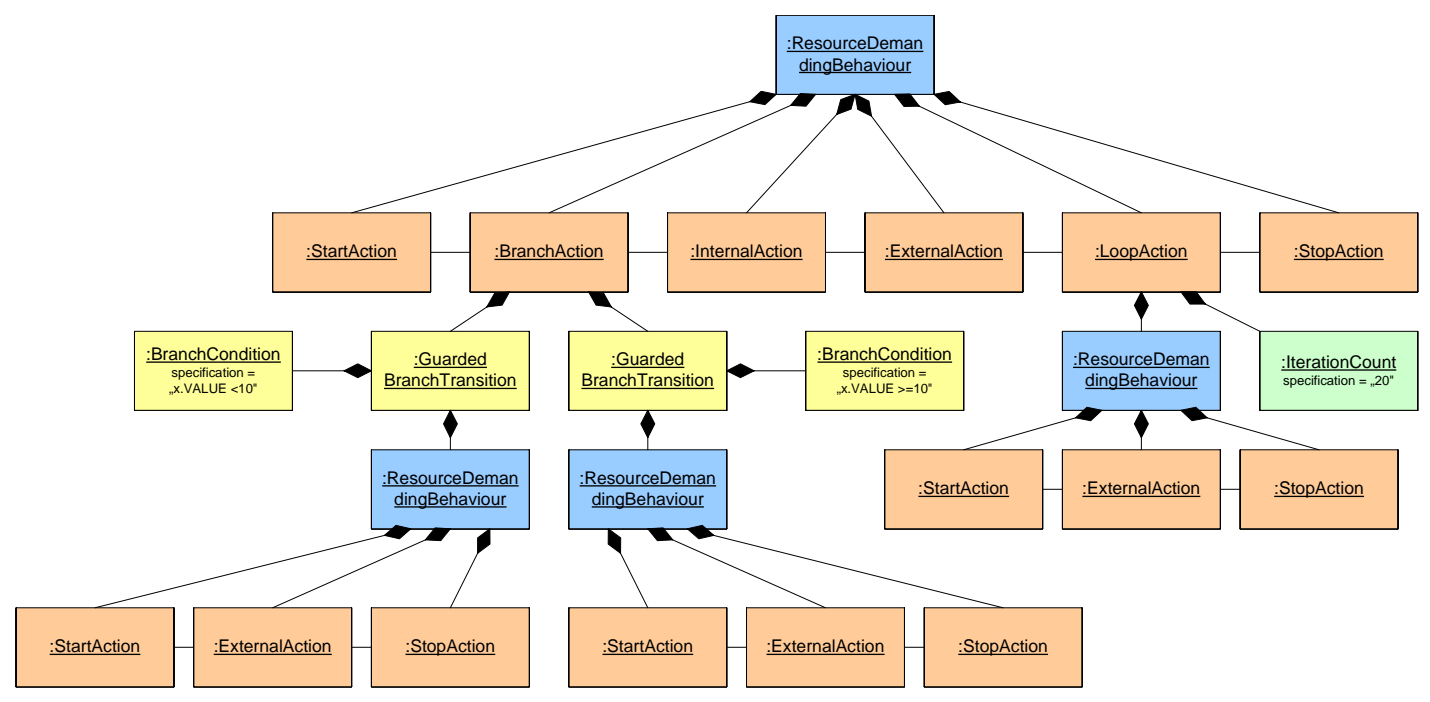

Figure 3.16: Example Instance RDSEFF highlighting control flow concepts

Parametric Dependencies A major problem for component developers is that during component specification it is unknown how the component will be used by third parties. Thus, in case of varying resource demands or branch probabilities depending on user inputs, component developers cannot specify fixed values. However, to help the software architects in QoS predictions, the component developer can specify the dependencies between input parameters and resource demands, branch probabilities, or loop iteration numbers in SEFFs. If an usage model of the component has been specified by business domain experts or if the usage of the component by other components is known, the actual resource demands and branch probabilities can be determined by the software architect by solving the dependencies. In the following, examples for the specification of parametric dependencies in the PCM will be illustrated. Note that as a concrete syntax a more easily readible UML-based notation is used for the examples instead of the abstract syntax.

As the first example (Figure 3.17), the ResourceDemandingSEFF of the service HandleShipping from an online-store component is depicted. It has been specified by a component developer in a parametrised form. The service calls required services shipping a customer's order with different charges depending on its costs, which it gets passed as an input parameter. If the order's total amount is below 100 Euros, the service calls a service preparing a shipment with full charges (ShipFullCharges). If the costs are between 100 and 200 Euros, the online store grants a discount, so ShipReducedCharges is called. Orders priced more than 200 Euros are shipped for free with the ShipWithoutCharges service.

Once a domain expert specifies the value of the parameter costs, it can be derived which of the services will be called.

The second exmample (Figure 3.18) illustrate assigning a number of iterations to a loop in a parameterisable way. The illustration shows the ResourceDemandingSEFF of the service UploadFiles. It gets an array of files as input parameter and calls the external service HandleUpload within a loop for each file.

With the specified dependency to the number of elements in the input collection, the probability distribution of random variable $X_{i t e r}$ for the number of loop iterations in the ResourceDemandingBehaviour can be determined once the number of elements are known. If the dependency had not been specified, it would not have been known from the interfaces how often the required service would 


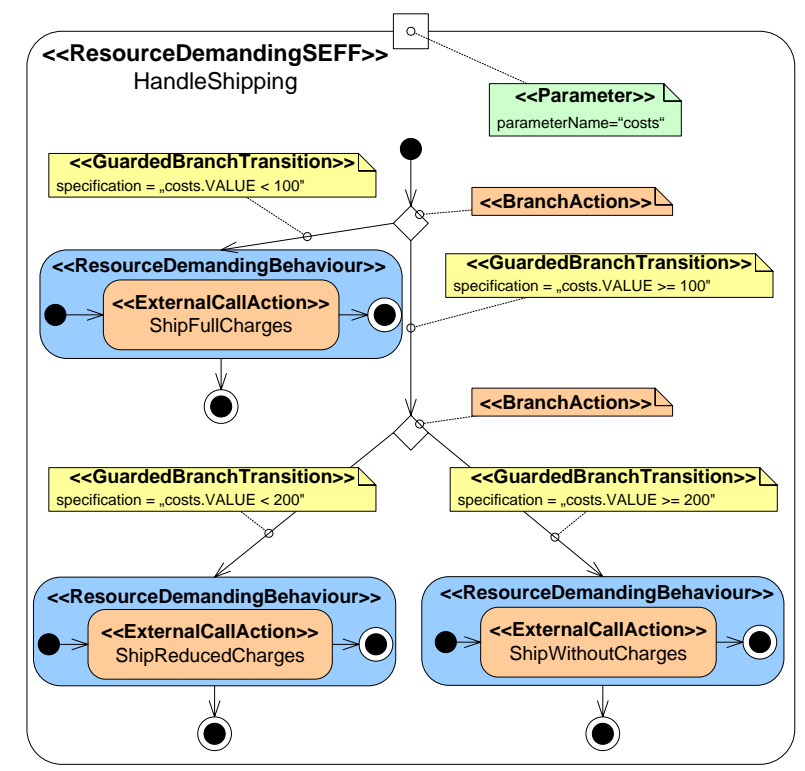

Figure 3.17: Branch Condition Example

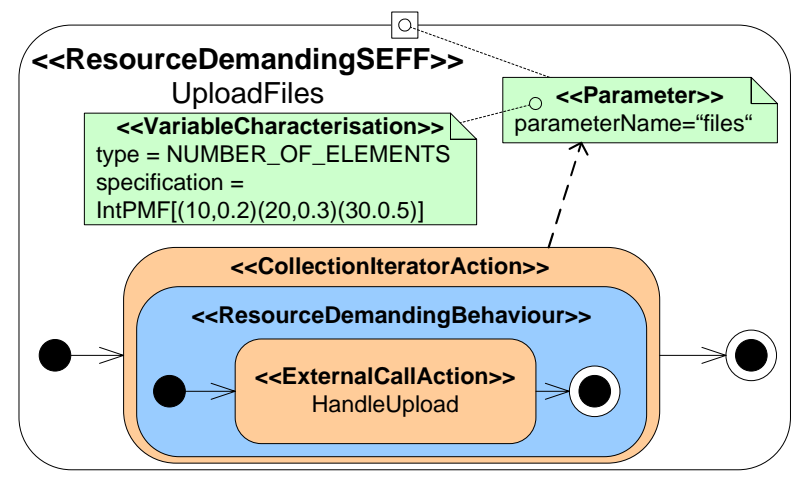

Figure 3.18: Loop Example 
have been called. Thus, with the specified PMF, a more refined prediction can be made for varying usage contexts. 


\subsection{Software Architect}

\subsubsection{Overview}

The tasks of the software architect are to retrieve components from existing repositories and connect them to build an assembly which is an essential part of the complete system. Connections are specified by using system assembly connectors to connect required roles of components with provided roles of other components. After connecting all components, the software architect puts the components into a system and defines the system provided and system required roles as well as the respective delegation connectors. The definition of a system and its boundaries is comparable to the definition of composite components. However, the difference is that composite components are built with the aim to use them in other composite components or assemblies. On contrary, systems are built to interact with other systems only. An overview of a system and its subconcepts is shown in figure 3.19.



Figure 3.19: A system and its assembly

Components can only be used in contexts as introduced in section 2.4. Hence, the software architect is responsible to introduce system assembly contexts in which a component is put. When a component is put into a context its roles also become part of the context. Such roles which are part of a context can be connected. For this, a required role in a specific context is connected to a compatible provided role in an other context. A single component can be put into several different contexts and can be connected differently in each of them. As mentioned in section 2.4, the introduction of multiple assembly contexts is important as they capture the different influence of component external calls in different contexts.

The defined assembly model is finally passed on the the system deployer who specifies the allocation of the components to middle- and hardware environments. The assembly model is the second essential part of a system and is described in detail in section 3.3. 


\subsubsection{Assembly}

An assembly is a set of assembly contexts containing component types from several repositories and a set of system assembly connectors connecting the components in their context. Conceptually, every system has exactly one assembly. An assembly is different from a composite component in its visibility for the system deployer. The inner structure of a composite component is hidden from the system deployer. Only the outer aspects of the component are visible for the system deployer which is mainly the component and its roles. Opposed to this, the system deployer has full access to the assembly contexts and the system assembly connectors. The rationale behind this difference in modelling is that a composite component should always look like any other component (besides for the developer of that component). The decision, whether a component's inner structure is build from scratch (i.e., as basic component) or by connecting existing components (i.e., using a composite component), is considered as an implementation detail. As a consequence the inner structure of any component is only visible to the component developer. Neither the assembler nor the deployer know about the inner structure. To be consequent this means that a composite component can not be allocated on more than a single runtime environment as this would mean that the system deployer has access to the composite components inner structure. This is different for an assembly. The component and their contexts as well as the system assembly connectors are visible and can be distributed in arbitrary ways by the system deployer on execution environments.

\subsubsection{Assembly Context}

As introduced above, the software architect uses assembly contexts to put components into a component assembly. Contexts support the multiple use of the same component type in several environments in an assembly.

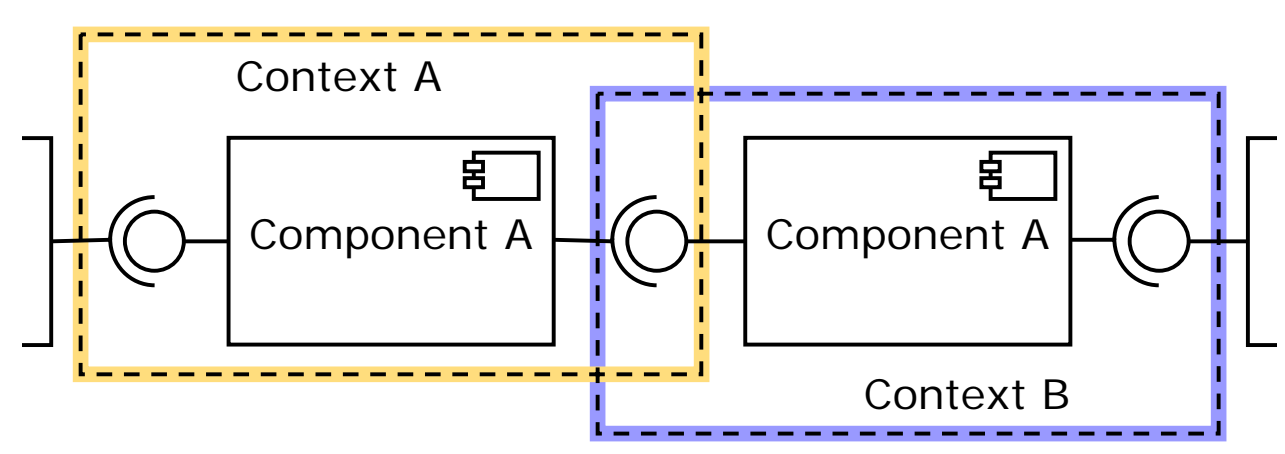

Figure 3.20: Component Assembly Context

The assembly context refers to exactly one component from an arbitrary available repository for which the context is applied. The component and its provided and required roles are affected by the context in which it is used. This can be indicated by deriving from the provided and required roles the corresponding provided and required context roles.

According to the principles of parametric contracts (see section 2.3, context roles represent the contextual influenced interfaces of the component in a given assembly context. 


\subsubsection{System Assembly Connectors}

After putting components into assembly contexts (from which provided and required context roles can be derived) they can be connected by using system assembly connectors. A system assembly connector connects a required role in of a component in a given assembly context with the provided role of a component in a different assembly context ${ }^{2}$. The meaning of the connector is that any call of the client component using the required role involved will be routed to the provided role of the server component.

Connectors are important entities when it comes to checking of interoperability classes. The minimum requirement a connector has to fulfil is that the interface of the provided role is a supertype of the required role. This automatically implies semantic conformance of the interfaces (compare section 3.1.2).

\subsubsection{System}

As mentioned in the overview, an assembly forms on of the important aspects of a system. A system consists of an assembly and an allocation as described in section 3.3.5. The first specifies how the components are connected with other components, the latter specifies how the components and connectors are mapped to hardware and middleware environments. Systems can be seen as special kind of composite components - with the visibility differences mentioned above and the fact that an allocation is also provided. Systems are not supposed to be reused as components are. The are assumed to be coupled by using special techniques for system integration.

\subsubsection{System Roles}

As components, also systems can specify that the offer the functionality of a specific interface or that they require functionality of a specific interface. Analogous to the component roles, the PCM defines system provided and system required roles. The semantics corresponds to the semantics of the roles of a complete component type. The system offers the functionality specified in the provided interfaces if all requirements of the system are met. If they are not met, only a subset will be offered. The semantics of the required interfaces is that a system may call other systems using a required role. It can not call other services than those defined in the system required roles. Using parametric contracts (see section 2.3) for functional dependencies, the actuall demand or the actual provided functionally can be derived (which would result in a system context role, but as it can be fully derived, it is not part of the PCM specifications).

\subsubsection{System Delegation Connectors}

Systems can have delegation connectors, just like composite components. The delegation connectors are used to route calls to the system interfaces to the desired destination. As composite component delegation connectors there are also two types of system delegation connectors: provided and required. Provided system delegation connectors route calls to system interfaces to components in the assembly which are responsible to process the requests. System required delegation connectors route calls of components in the assembly, which are not processed in the current system, to system required roles. Hence, they can be used to model calls to other systems.

\footnotetext{
${ }^{2}$ Using the derived context roles as concept, we can say, a system assembly connector connects a required context role and a provided context role
} 


\subsection{System Deployer}

\subsubsection{Motivation}

To execute an application specified by a component assembly, components and connectors have to be allocated to different hardware and software resources, which provide the required infrastructure. Servers, clients, or any other kind of systems are set up with the required operating system and middleware. Components are installed on the systems and configured so that they can run in this environment. Computers are connected by networks enabling the communication needed by the components. The whole process of setting up the infrastructure, allocating components, and configuring the system is handled by the deployer as introduced in section 2.1.

For QoS analyses, it is required that the deployment of the software architecture is specified in advance, since it has a major influence on QoS attributes, such as performance and reliability. For example, the response times of a component's services will be shorter when it is deployed on a machine with a $3 \mathrm{GHz}$ processor instead of a machine with a $1 \mathrm{GHz}$ processor. With the specification of the execution environment with its hardware and software resources and connections, and the component allocation, several QoS attributes can be predicted. So, deployers are able to try different deployment scenarios to find the optimal configuration for a software architecture. In many cases, such a procedure can save a lot of work and cost, since bottlenecks can be discovered early and hardware will not be oversized.

In the context of the PCM, we currently provide a basic model for the description of resource environments and allocation of components. In the following, we describe how these concepts can be used to specify new resource types that form an execution environment. Furthermore, we introduce allocation contexts that allow us to allocate components to multiple hard and software nodes. For the future, it is most likely that the model described here will be extended to allow a higher accuracy in terms of modelling as well as prediction results.

Section 3.3.2 describes the responsibilities and duties of deployers. In section 3.3.3, we describe what kinds of resource types we model and how they can be used. Section 3.3.4 shows how the PCM in combination with a fixed set of resource types can model an execution environment. In section 3.3.5, we describe how components are allocated on resource containers and how they can access the available resources. Finally, section 3.3.6 sums up open issues and assumptions of our model.

\subsubsection{Responsibilities of the Deployer}

Mainly, the resource environment is in the deployer's responsibility. This includes the specification of resource environments as well as the installation of a component-based application or the setup of a new environment. Deployers are assumed to be experts in the area of component deployment (allocating software components to different hard- and software nodes) and the configuration or creation of an environment, that enables the system to fulfil its extra-functional requirements. Deployers are responsible for:

- Definition and description of the resource environment. This includes the specification of hardand software resources, their properties, and their interconnections.

- Allocation of components to different resources.

Deployers are not only concerned with the specification of the resource environment and component allocation, but also with the realisation of the actual system setup. However, as these are two different 
tasks, they might not be performed by a single person only. For example, an application for the mass market might have a set of typical deployment scenarios defined by members of the development team, but the setup will be accomplished by the customers themselves.

To specify the resource environment in the PCM, deployers use resource containers. A resource container represents a part of the real world that can host components, for example an application server or client computer. It holds a set of different resources, such as processors, hard disks, or thread pools. Each resource conforms to a certain resource type that discribes a class of resources with common properties. If a component is allocated on a resource container, it has access to all resources the container provides.

\subsubsection{Resource Types}

A resource type describes the common properties of a class of resources. For example, a processor type could be used to describe different CPUs, e.g. with a different clock speed or a different architecture. The concept of resource types allows a flexible specification of different kinds of resources that might occur in a real world scenario. Component developers and deployers agree on a common set of resource types that is specified within a so-called resource repository.

We distinguish passive and processing resources. Passive resources can only be owned by a process or thread, while processing resources do some work by themselves and offer processing services. A scheduler might decide, which process is handled next by the processing resource. CPUs and hard disks are typical processing resources, while connections to a database or a block of memory are passive resources. Communication links are a special kind of processing resource type used to describe connections between different resource containers.

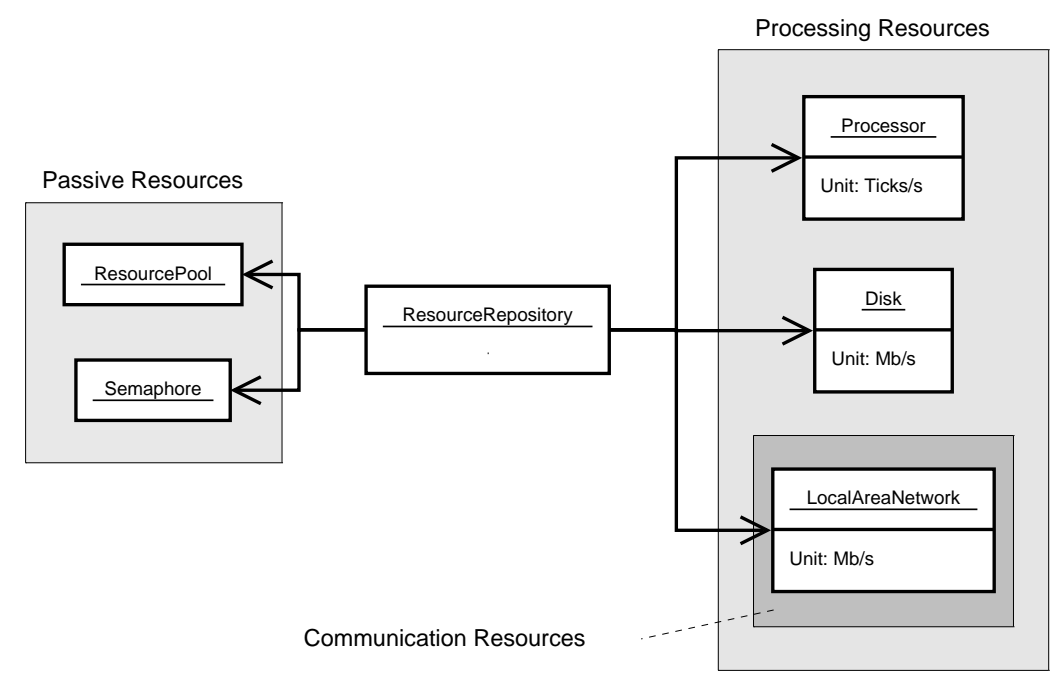

Figure 3.21: Basic instance of a resource repository.

Figure 3.21 shows an initial instance of a resource repository. It contains a set of passive and processing resources. The processing resources themselves are subdivided into plain processing resources and communication resources. The latter can only be used to connect two different resource containers. In this example, resource pools and semaphores are the only passive resources. In the following, we describe the different resource types in more detail.

Resource pools manage a limited set of resources of the same type. Typical examples are database connection pools and thread pools. A process or thread can fetch one database connection, use it to 
read or update some of the database entries and then return it to the pool. If no database connection is available, the process will block until one is available in the pool.

Semaphores are the most basic kind of passive resources. They can be used for synchronisation and limiting access to a resource. Basically, a semaphore is an integer value with an acquire (or $p$ ) and release (or v) operation. Intuitively, the value of a semaphore indicates how many instances of a resource are available. If the semaphore is greater than zero, the acquire operation reduces the semaphore counter by one and continues the execution. Otherwise, it waits until the counter is greater than zero. The testing and setting of a semaphore's value has to be atomic (i.e., it must not be interrupted). The release operation increases the counter by one, which must be atomic as well, and awakes the waiting threads or processes.

Acquire and release actions are used for semaphores as well as for resource pools and can be directly modelled in the service effect specification (see section 3.1.4).

Processors and disks are classical processing resources. They are available in every desktop and server computer system and provide the basic computational and storage functionalities. In figure 3.21, the processing rate of a CPU is specified as the number of cycles per second. Another possible metric is the number of instructions per second.

Disks are used to permanently store and to retrieve data if needed. In figure 3.21 , we indicate the speed of a disk (or data storage) in megabytes per second. In our case, we assume that the rate is the same for read and write operations.

For communication resources, we consider any kind of network connection. The rate or throughput of a connection is specified in megabytes per second. This resource type can be used to model most of the common networks. For example, a wireless connection between two nodes can be described as an ethernet connection with a throughput of $11 \mathrm{MB} / \mathrm{s}$.

The resource types described here can be considered as a basic set, which has to be extended and refined in future. Next, we describe how these resource types can be used to specify an execution environment.

\subsubsection{Resource Environment}

In the PCM, resource environments are described by a set of resource containers and connections between them. A resource container provides a set of processing and passive resources to the components it hosts. It represents a physical entity such as a server, a desktop computer or an element on a higher level like application servers or web browsers.

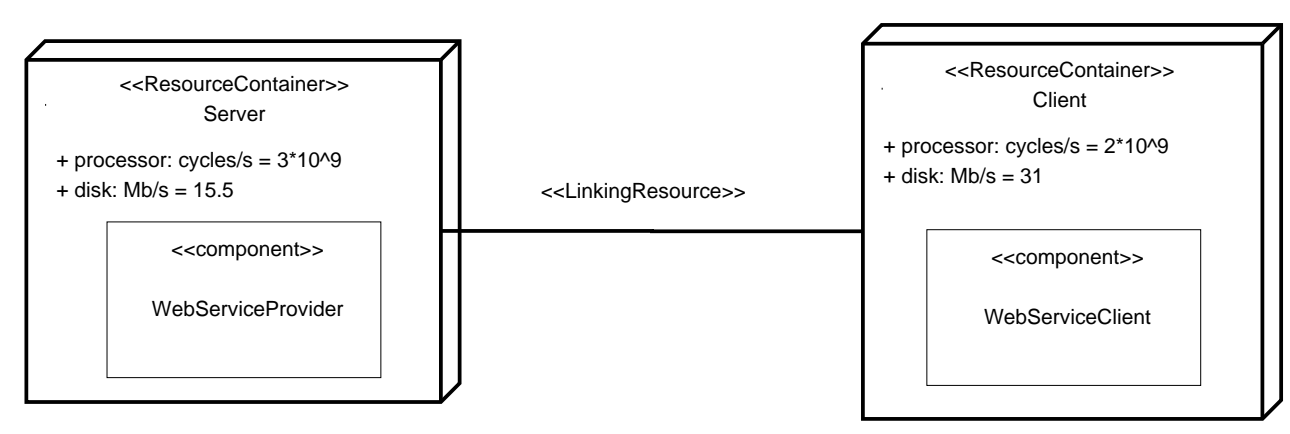

Figure 3.22: Simplified example of a resource environment.

Example 3.2 (Resource Environment). Figure 3.22 shows a simplified view on a resource environment. The depicted system consists of two resource containers, a server and a client, and a linking resource 
between them. The figure also shows the allocation of two components, a WebServiceProvider and a WebServiceClient. Figure 3.22 is a structural view of the resource environment. For each container, a processor and a disk are specified. Both have different performance values for the resources they provide. For instance, the processor of the server has a clock frequency of $3 \mathrm{GHz}$, while the client has a clock frequency of $2 \mathrm{GHz}$.

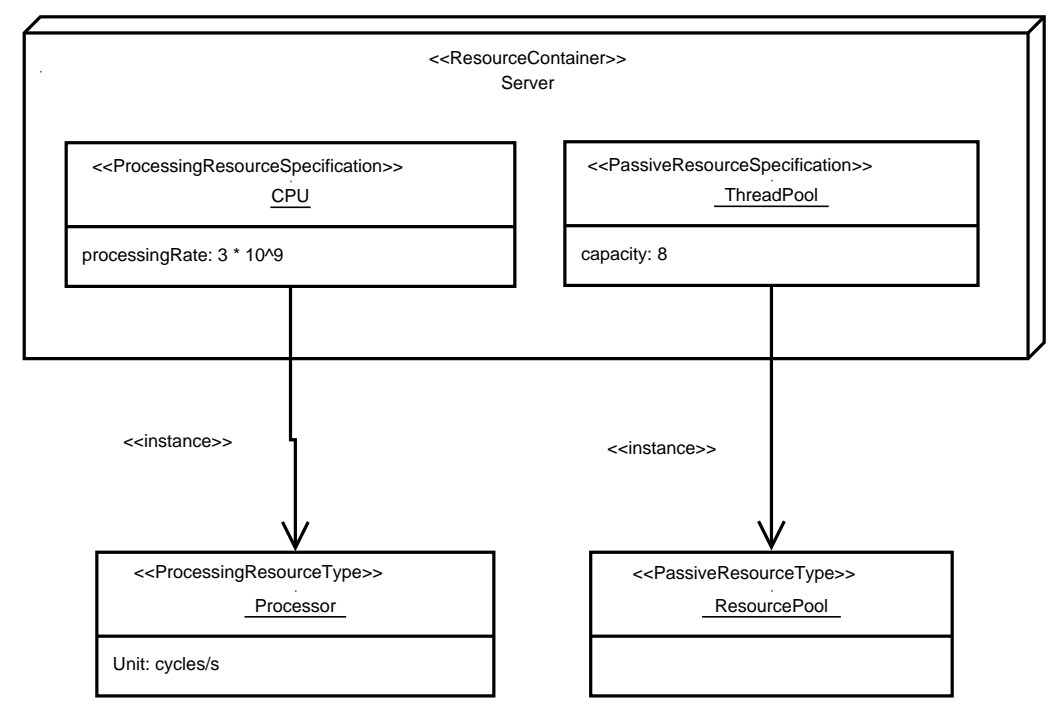

Figure 3.23: Specification of Resources of a Container.

Figure 3.23 shows the resource specification in more detail. The server contains a CPU and a ThreadPool. Both are described by ProcessingResourceSpecifications, which characterise the QoS relevant attributes of a resource and relate it to a resource type. There is a CPU with a processing rate of $3 \mathrm{GHz}$ and a thread pool that limits the degree of concurrency within the system. The CPU is a processing resource of the type Processor. The type also specifies the units of the CPU's processing rate. The thread pool is a passive resource with a capacity of eight threads. The thread pool is of the type ResourcePool, which is depicted by an association to the type instance. For sake of simplicity, we omitted the modelling of any kind of data storage and hard disks at this point.

Passive and Processing Resources Resources are divided into processing and passive resources, whose concepts are elaborated in the following.

Active resources are those which perform tasks on their own and thus can actively execute a task. This includes CPUs, hard disks, and network connections. As these resources always do some kind of job processing, we call them processing resources.

Passive resources on the other hand can be owned by a process or thread for a certain period of time. A passive resource has to be acquired to be accessed. Since passive resources can be limited, processes or threads might have to wait until a resource becomes available. Typical examples of passive resources are connection pools and thread pools. The acquisition and the release of a passive resource has to be represented in the SEFFs, which describe the control flow of a component (see page 50). If a component requires access to a limited resource, it first has to acquire it using the AcquireAction. After it has finished its operation, it has to release the resource using the ReleaseAction. The semantics of a passive resource with its AcquireActions and ReleaseActions is based on the semantics of semaphores. 


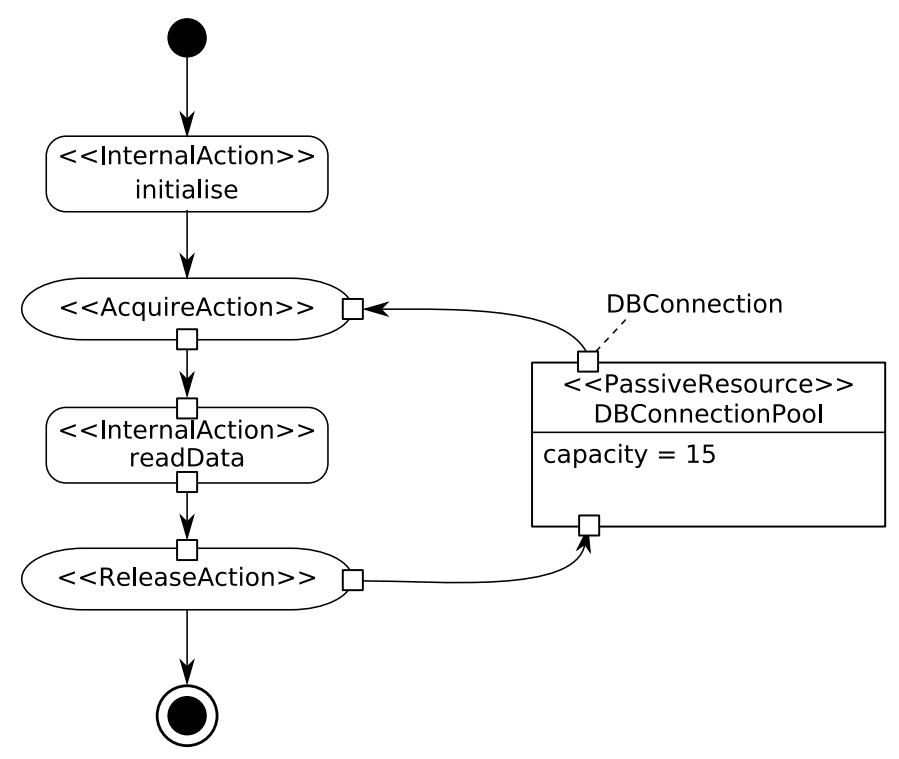

Figure 3.24: Example of a SEFF using a passive resource.

Example 3.3 (Passive Resource). Figure 3.24 shows a simple SEFF that uses a passive resource. First, the SEFF performs some initialising actions that are captured in the InternalAction initialise. Next, an AcquireAction is invoked to get a connection to the database. The capacity attribute of the DBConnectionPool indicates that there are 15 connections to the database available. If no connection is left, the AcquireAction blocks the current thread until a database connection is returned to the pool. The DBConnection object is then passed by the AcquireAction to the InternalAction readData, which reads some entries from the database. Finally, the ReleaseAction returns the connection object to the $\mathrm{DBC}$ nnectionPool allowing other processes to use it.

Example 3.3 shows how a passive resource is used by a SEFF. The object received from the DBConnectionPool is passed from one action to another. Within the actions, the object can be used. So, a passive resource can be owned and used by a process or thread for a certain period. Opposed to that, active resources cannot be owned. A scheduler decides which thread or process will be handled next by a processing resource.

\subsubsection{Allocation Context}

After introducing different resource types and means to specify execution environments, which provide the infrastructure to an application, components have to be allocated on the available resource containers. For this purpose, the PCM uses the allocation context. In section 3.2.3, we described how a component is integrated in a system assembly using assembly contexts. The idea of allocation contexts is similar. Each component integrated in an assembly might be allocated on multiple resource environments. Thus, for each component in an assembly context, there can be multiple allocation contexts that place the component on different resource containers. For example, a possible alternative of the allocation in figure 3.22 is shown in figure 3.25 .

Figure 3.26 shows a simplified instance of the PCM that realises the allocation shown in figure 3.25. The allocation context is an association class that links a component to a resource environment. The allocation context allows to specify the placement of the same component on multiple resource environments. In reality, a copy of the component is created for each machine. Furthermore, the allocation 


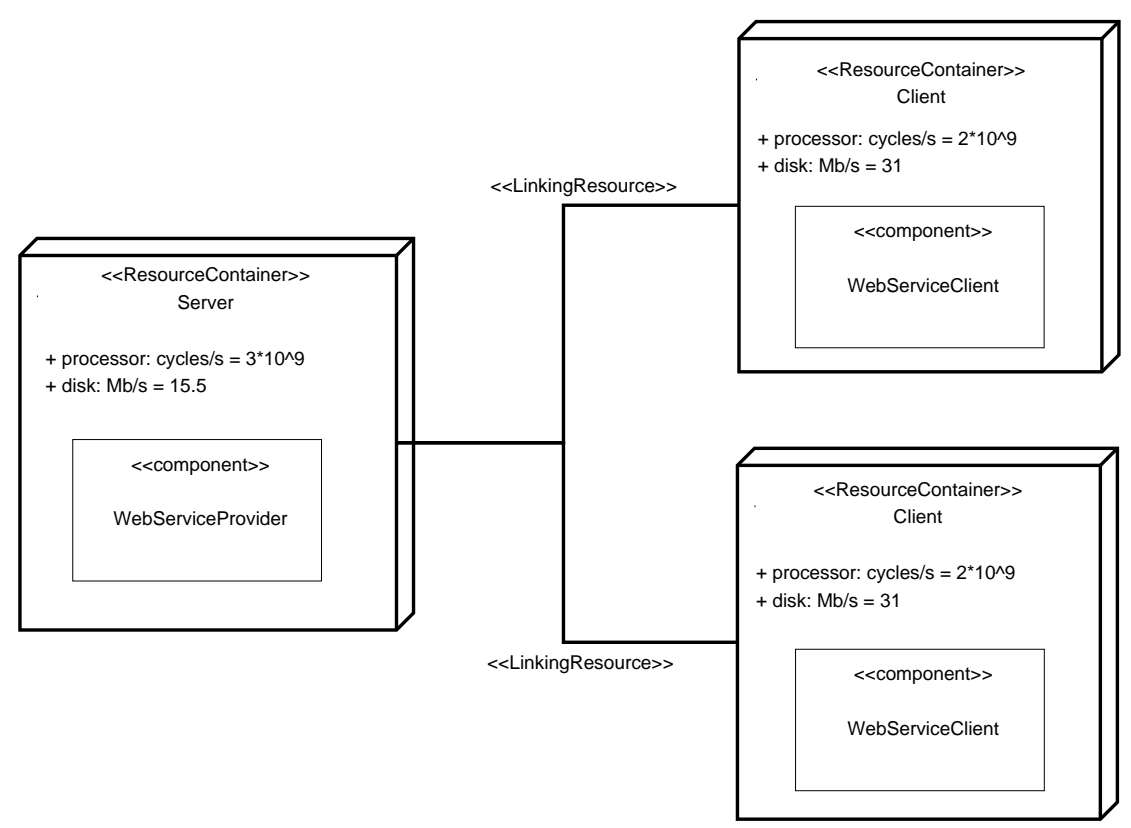

Figure 3.25: Alternative allocation for figure 3.22.

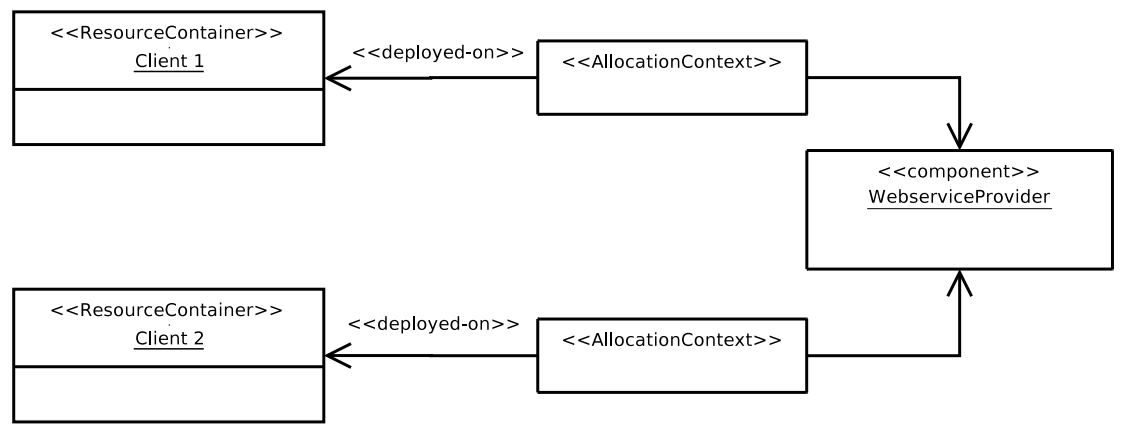

Figure 3.26: Allocation of a component on multiple resource environments (simplified). 
context stores QoS related information that depends on the resources used by a component. For example, if an internal action of a component uses 5000 cycles on a Proces sor resource, this can be transformed to an execution time of $1 . \overline{6} \mu \mathrm{s}$ for a processor with $3 \mathrm{GHz}\left(1 /\left(3 * 10^{9} \mathrm{~s}^{-1}\right) * 5000\right)$. As the execution time of internal actions depends on the resources the component is allocated on, these information are handled by the allocation context.

In the PCM, resource environments are described using resource containers holding an arbitrary number of processing and passive resources. Linking resources connect resource containers with each other and provide a communication resource for sending data from one container to another. Resource types can be used to specify which kinds of passive, processing and communication resources exist. Components that are integrated into an assembly can be allocated on resource containers using allocation contexts. These allow to allocate one component on multiple resource containers and store QoS relevant information, which depends on the container, independent of the component. So, the PCM provides a complete infrastructure to specify the environment of an application and its allocation. However, there are a lot of open issues that need to be addressed in the future. We will discuss some of them in the following.

\subsubsection{Open Issues and Future Work}

So far, the PCM does not support the modelling of hierarchical resource containers. This is a major limitation for deployers, since they cannot model different software layers running on the same machine. For example, virtualisation of operating systems cannot be specified. Furthermore, it is not possible to describe systems that contain multiple components that are placed on different software layers, e.g. operating system and application server, but on the same machine.

Another limitation stems from the modelling of linking resources. At the moment, we only allow a single linking resource between two resource environments with one specification. Thus, scenarios in which two hardware nodes are connected by multiple links, e.g. LAN and wireless LAN connection cannot be modelled. Furthermore, it is not possible to explicitly allocate connectors between components to linking resources. With only one connection between two containers, this can be done automatically using direct links only. However, if multiple connections are allowed the allocation of connectors must be modelled explicitly. The same problem arises when indirect communication between containers is allowed. In this case, the communication path between components is ambiguous even with only one connection between two containers.

In section 3.3.3, we described how to specify new resource types. Even though this provides a high flexibility, it requires component developers and deployers to agree on a common set of resource types. For scientific purposes, this is feasible. However, we need to integrate a standardised set of resources into our model so that there are no mismatches between the specifications of different parties. As the modelling of execution environments is not as elaborated as other parts of the PCM, we left the specification of resources open for the time being. For the future, we plan to fix the available set of resources.

Also, the specifiable properties of the resource types are limited. So, if a new resource type has additional attributes that have to be specified, this cannot be described. For example, queues could be introduced as a passive resource. Usually, queues are used for asynchronous communication between multiple processes and threads. One process puts a message or any data into the queue while another process reads it. A producer-consumer system is a common example for such a scenario. A special application for queues can be found in combination with active objects [28]. Instead of calling a method of an active object directly, the call with its parameters is placed in a message queue. The scheduler of 
the active object fetches the messages from the queue and processes them. Queues do not only have a capacity as all passive resources do, but also require an attribute which specifies the order in which its items are processed, like LIFO or FIFO. This is not possible so far.

Furthermore, multicore processors and multi-processor systems are becoming more and more common forming new challenges for the PCM, hence we need appropriate mechanisms to specify the QoSrelevant aspects of these systems. For example, the number of memory buses and caches has a significant influence on the performance of a multithreaded application. So, specifying two processors with the same properties might not be sufficient, as these processors share other important resources that are not modelled explicitly. 


\subsection{Domain Expert}

\subsubsection{Overview}

Business domain experts participate in the development of any larger software system. This role has special knowledge and experience in the business domain (e.g., automobile, banking, etc.) of the system being developed. However, domain experts usually have no or only a limited technical background. They mainly participate in the development process during feasibility studies and requirements analyses and help in specifying the functionality and business logic of the system. Therefore, they have to interact closely with the system architects, who have a technical background and are able to tailor their requirements to a component-based software architecture.

For early QoS analyses, domain experts assist system architects in specifying the user interaction with the system. As they are familiar with the business domain and the targeted end-users, they should best be able to specify the anticipated usage scenarios and workloads of the system. The usage specifications may be based on experiences with similar legacy systems or on market analyses of the business domain. In the PCM, the usage specification consists of usage models (see Section 3.4.2), which are similar to UML use cases with attached UML activities. They additionally contain stochastical information (e.g., probabilities of choosing a branch in an alternative) and the notion of workload to characterise the number of users in the system, which is especially relevant for performance predictions. Usage models may be refined with a parameter model (explained in Section 3.4.3) to characterise the data values passed to component services by users.

\subsubsection{Usage Model}

An instance of the PCM usage model specifies user interactions with a system. It describes which services are directly invoked by users in specific use cases and models the possible sequences of calling them.

\subsubsection{Example}

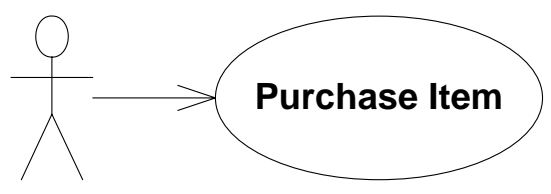

(a) Example UML Use Case

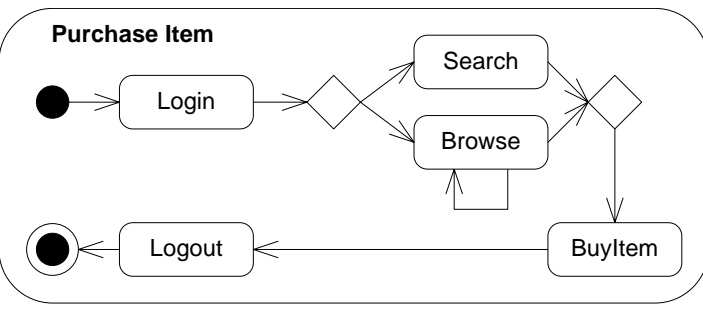

(b) Example UML Activity

Figure 3.27: Example UML diagrams for using an Online Shop

Example 3.4 (Usage Model). For a first overview, Figure 3.27 shows a UML use case diagram and corresponding UML acitivity for using an online shop. Users log in to the shop, either search or browse in the shop's catalog, then buy items, and finally log out. Figure 3.28 shows the corresponding PCM usage model instance of this scenario. In this example figure, the concepts were illustrated with UML activities, where the stereotypes (denoted by $<<$ stereotype $>>$ ) refer to classes in the PCM. This simple usage model instance serves as a running example for the rest of this section. 


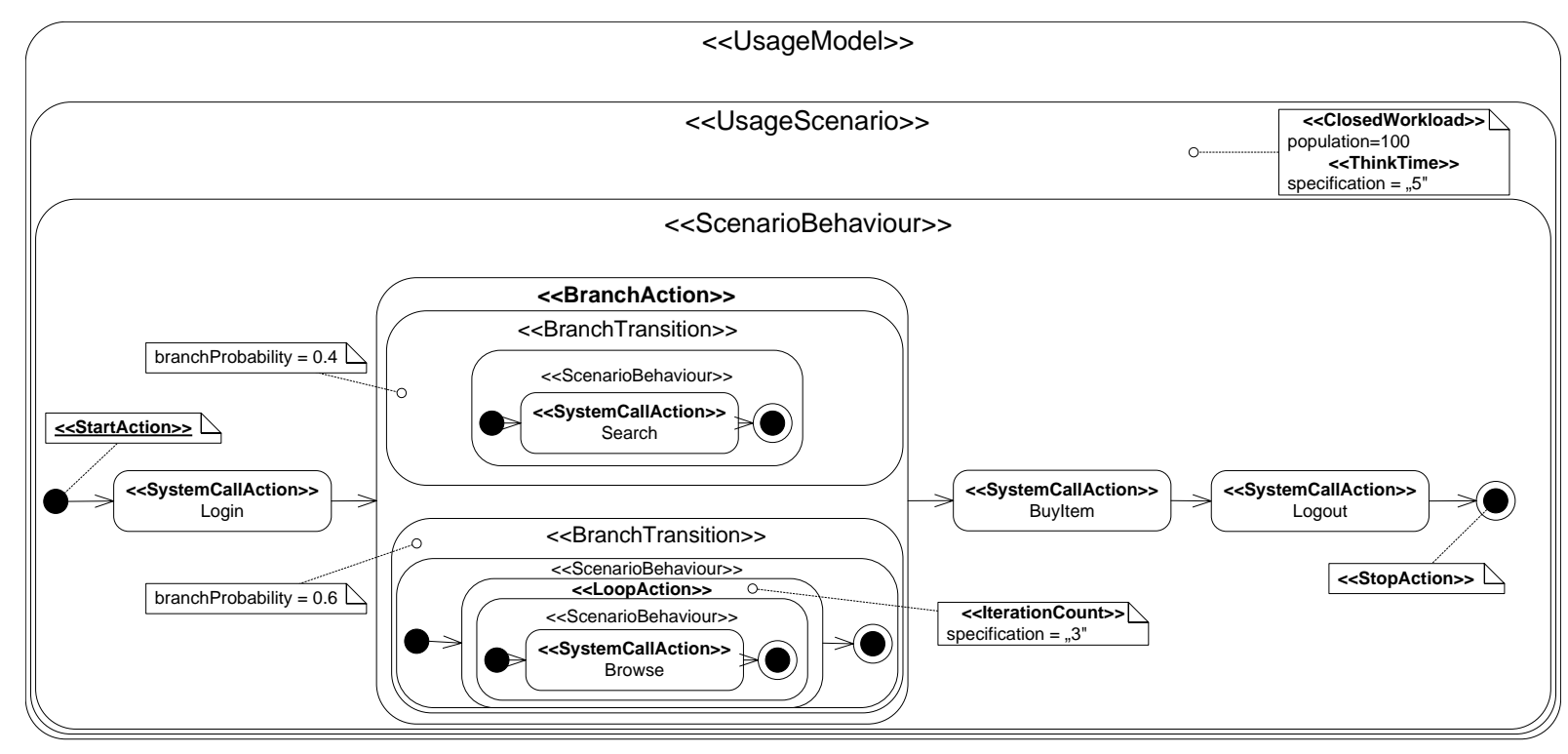

Figure 3.28: Example Usage Model for an Online Shop

An usage scenario consists of i) a workload to model the frequency of user interactions and ii) a scenario behaviour to model the steps of service invocations by users. In this example, the workload is a closed workload (upper right corner of Figure 3.28) and specifies that 100 users (population) execute the scenario behaviour. Each user executes the actions specified in the behaviour from the start action to the end action. After reaching the end action, the user reenters the behaviour at the start action after 5 seconds (think time). The number of users in the system is fixed to 100 in this scenario.

The actions inside the behaviour either model flow constructs (start, stop, branch, loop) or user invocations of services available in system provided roles (Login, Search, Browse, BuyItem, Logout) (also see Section 3.2.6). Like in the UML diagrams before, users first log in to the online shop and then either search directly for an item via a search interface or browse the shop's catalog to find an item to buy. This alternative is modelled with a branch action and the probabilities of search and browsing are specified as $40 \%$ and $60 \%$ respectively. Browsing the catalog is modelled as a loop with three iterations, as it is assumed that users need three clicks to find the item they want to buy. After browsing or searching is finished, the user continues with buying the selected item, and finally the logging out from the shop.

Note, that usage models are completely decoupled from the inner contents of a system (see Section 3.2.5), which consists of an assembly (see Section 3.2.2) and a connected resource environment (see Section 3.3.4). The usage model only refers to services of system provided roles. It regards the component architecture (i.e., the assembly) as well as used resources (i.e., hardware devices such as CPUs and harddisks or software entities such as threads, semaphores) as hidden in the system. Thus, the usage model only captures information that is available to domain experts and can be changed by them. Resource environment and component architecture may be changed independently from the usage model by system architects, if the system provided roles remain unchanged.

\subsubsection{Structure}

The meta model for usage modelling in the PCM is described with more detail in the following (see Figure 3.29). A usage model consists of a number of usage scenarios. Each usage scenario is intended 
to model a use case of the system and the frequency of executing it. Thus, a usage scenario contains a Workload to model execution frequency and a ScenarioBehaviour to model a use case.

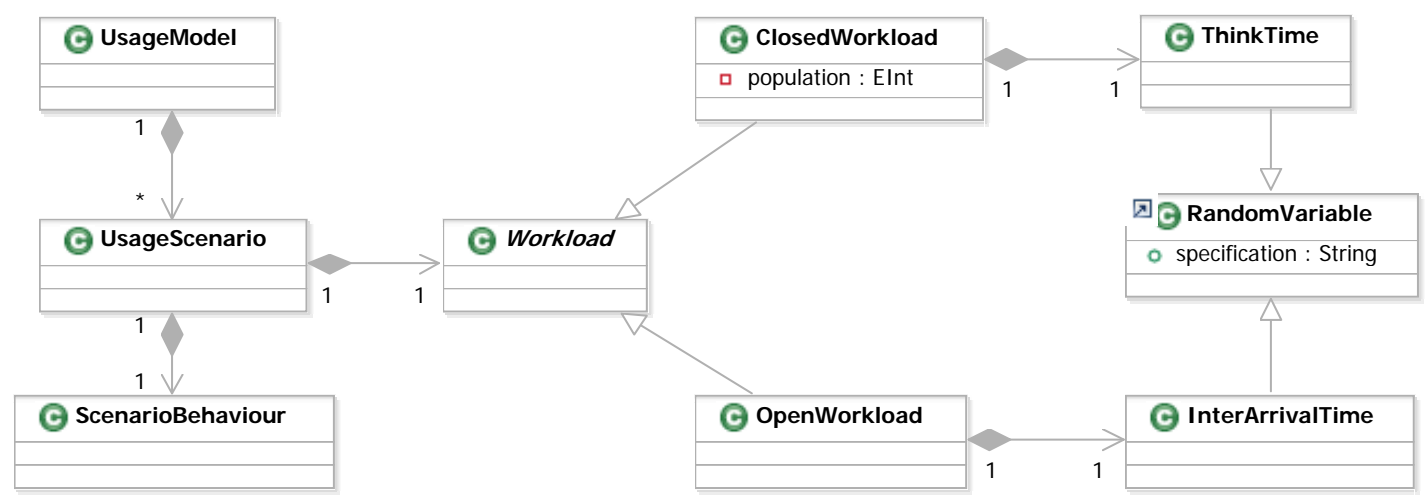

Figure 3.29: Usage Model: Scenario and Workload

Modelling workloads in the PCM is aligned with performance models such as queueing networks [29] or stochastic process algebras [30] as well as the UML SPT profile [31]. Therefore, open and closed workloads can be specified. An open workload models an unbounded (thus open) number of users entering the system with a specific inter-arrival time as a random variable (e.g., a new customer arrives each 0.5 seconds) and leaving the system after executing their scenario. A closed workload models a bounded (thus closed) number of users entering the system, executing their scenario, and then re-entering the system after a given think time, which can be specified as a random variable (see Section 2.5).

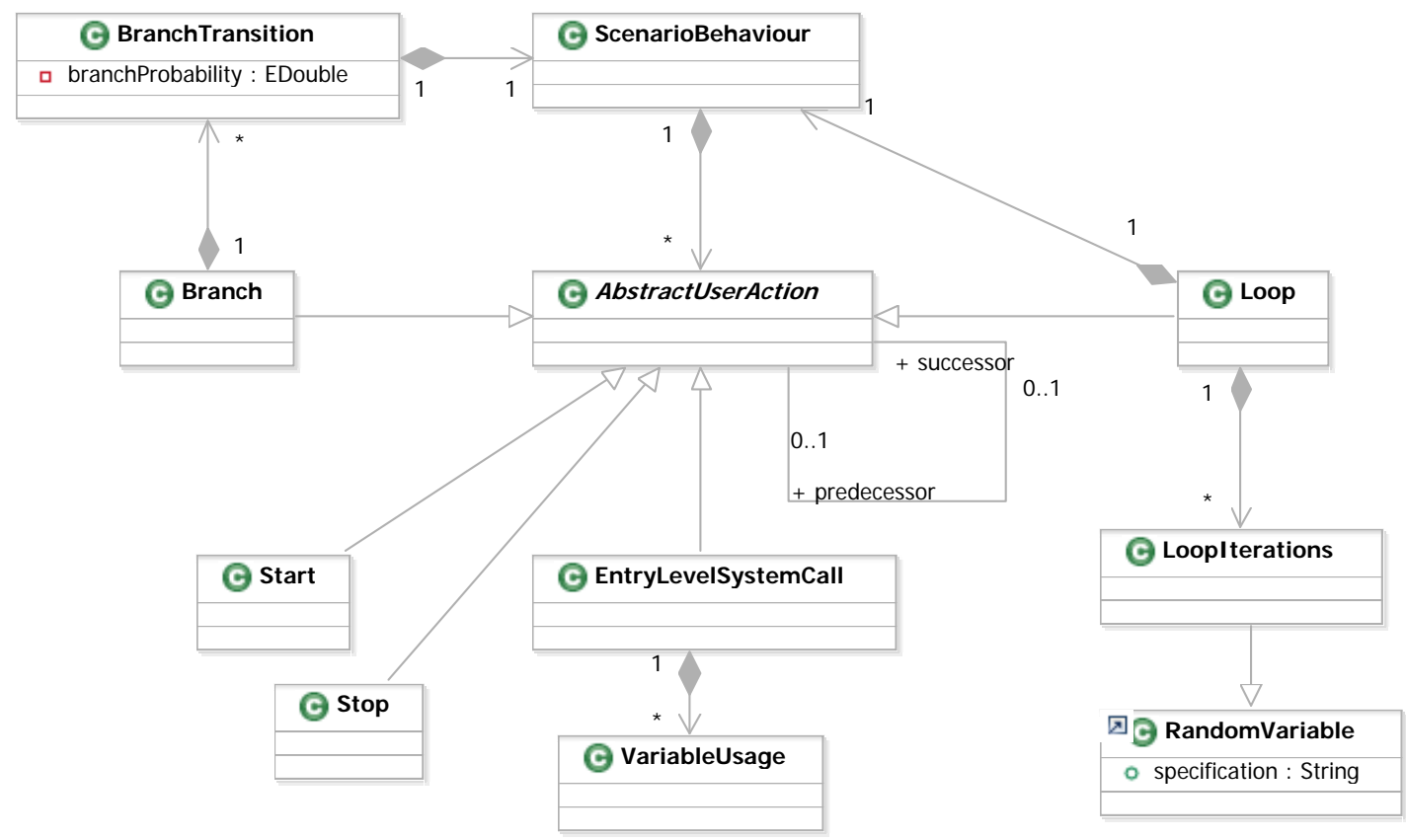

Figure 3.30: Usage Model: Scenario Behaviour

Modelling scenario behaviours in the PCM (Figure 3.30) is similar to modelling resource demanding behaviours in SEFFs (see Section 3.1.4). However, SEFFs contain notions of resource usage, while the 
language for usage scenarios is reduced to concepts familiar to domain experts, and does not refer to resources.

ScenarioBehaviours contain a number of user actions. Within a scenario behaviour, the flow of actions can be described as follows: Each AbstractUserActions references at most one predecessor and one successor. StartActions initiate a scenario behaviour and contain only a successor, while StopActions end a scenario behaviour and contain only a predecessor. Notice, that the start and stop actions in the example above (Figure 3.28) follow this pattern.

Loops can be modelled to describe user actions that are repeated multiple times (e.g., searching for an item in a online store by repeatedly entering search terms, or repeatedly checking the latest status of an online auction). Loops over user service invocations are modelled with LoopActions, which are attributed with the number of iterations and contain inner ScenarioBehaviours to model loop bodies. These loop bodies may consist of multiple actions or even nested loops themselves. In the example (Figure 3.28), the browse action is called within a loop three times. It is additionally possible to specify the number of loop iterations with a probabilty distribution instead of a constant value to allow more fine-grained modelling (see Section 2.5).

Notice that the chain of user actions in a scenario behaviour must not contain cycles to model loops, i.e., an action referencing another action as its successor and predecessor. Instead, loops always have to be modelled explicitly with loop actions. This explicit modelling eases the later analyses, as it arranges actions hierarchically in a tree structure, which can be analysed by standard tree traversal algorithms.

Most often, users have multiple choices to continue their interaction with the system. For such cases, the usage model offers branch actions, which are able to split the user control flow with an XOR-semantic and allow different successors to a single user action. A probability of executing each branch transition can be specified. In the example (Figure 3.28), users first log in to the system and then have the choice to either search the shop with a probability of $40 \%$ or browse in the shop's catalog with a probability of $60 \%$. Branchtransitions contain inner ScenarioBehaviours to model the content of a branch. With this kind of modelling, additional merge actions for reconnecting two branches are not needed, as the control flow continues with the successor of the branch actions once the end action of the the branched behaviour is reached. Forks of user behaviour (i.e., splitting the flow with an ANDsemantic) are not allowed so far, as it is assumed that a single user only executes the services of the same system subsequently but not concurrently.

Besides these control flow constructs, actual service invocations to the architecture are modelled by EntryLevelSystemCalls. They refer to services in system provided roles (see Section 3.2.6), which are connected to component services directly visible to the users. Inner component services, which are only called by other components cannot be referenced from the usage model.

\subsubsection{Related Work}

The PCM usage model has been designed based on meta models such as the performance domain model of the UML SPT profile [31], the Core Scenario Model (CSM) [32], and KLAPER [33]. It is furthermore related to usage models used in statistical testing [34]. Although the concepts included in the PCM usage model are quite similar to the modelling capabilities of the UML SPT profile, there are some subtle differences:

- The usage model is aligned with the role of the domain expert, and uses only concepts known to this role. It is a domain specific language, whereas the UML SPT performance domain model is a general purpose language that includes information, which is usually spread over multiple developer roles such as the component assembler and the system deployer, so that a domain expert 
without a technical background could not specify an instance of it. Nevertheless, domain experts should be able to create PCM usage models with appropriate tools independently from other developer roles, because such models only contain concepts known to them.

- The number of loop iterations is not bound to a constant value, but can be specified as a random variable.

- The control flow constructs are arranged in a hierarchical fashion to enable easy analyses.

- Users are restricted to non-concurrent behaviour, as it is assumed, that users only execute the services of a system one at a time.

- System service invocations can be enhanced with characterisations of parameters values, as described in Section 3.4.3.

\subsubsection{Parameter Model}

\subsubsection{Motivation}

Parameters of component services may have a significant impact on the perceived performance and reliability of a component-based systems. It can be distinguished between

- Input Parameters: which are passed to a component service by its clients (users or other components)

- Output Parameters: return values, which are sent back to clients by a service after finishing its execution

- Internal Parameters: which can be global variables or configuration options of a component

All of these forms of parameters can cause different influences on the QoS properties of a system:

- Resource Usage: Parameters can influence the usage of the resources present in the system executing the component. For example, the time for the execution of a service that allows uploading files to a server depends on the size of the files that are passed as input parameters. In this case, the parameter alters the usage of the storage device. Another example would be a service for sorting items within an array. The duration of executing the sort service would mainly depend on the size of the array passed to it. Thus, the parameter would alter CPU usage.

- Control Flow: SEFFs (see Section 3.1.4) describe how requests to provided services are propagated to other components. The transition probabilities or number of loop iterations in SEFFs can depend on the parameters passed to a service. For example, a component service might provide clients access to a number of databases, thus communicating with several database interfaces as required services. This service would call different required services depending on the input parameter passed to it. Thus, the transition probabilities in the SEFF modelling the alternative to communicate with different databases would directly be linked to the input parameter. Another example could be a component service having a collection parameter, which would call another component's service subsequently for each item in the array. Such a situation would be expressed as a loop in a SEFF, and the number of iterations would directly be linked to the size of the array. 
- Internal State: Input parameters can influence the internal state of the component. The component state in turn may influence resource usage or control flow between components. Imagine a component allowing users to $\log$ in to a system, which stores user sessions as global variables. The later behaviour of other services of this component in terms of control flow propagation and resource usage could depend on which user is currently logged in. Thus the QoS properties of the component would be related to the internal parameter, which was created when the user logged in to the system. Although the influence of the internal state has been recognised by us, it is so far not modelled in the PCM and remains future work.

\subsubsection{Example}

Before describing the parameter model in detail, two short examples are shown in Figure 3.31 to give the reader a feel for the modelling capabilities. These examples extend certain actions from the usage model example in Figure 3.28 with parameter characterisations.

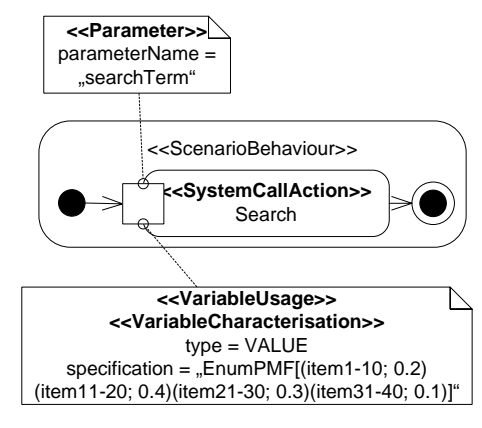

(a)

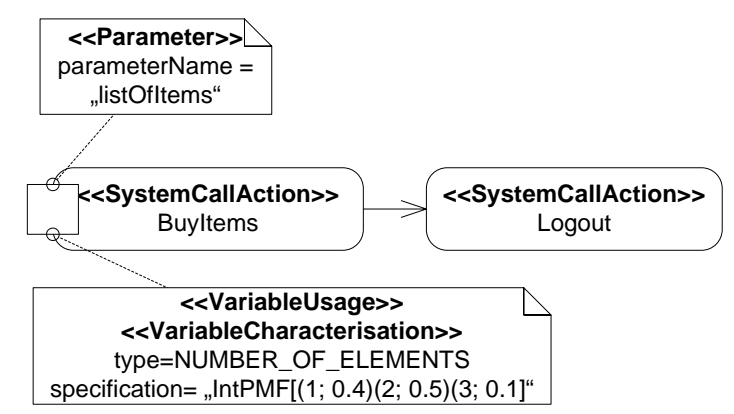

(b)

Figure 3.31: Parameter Characterisation Examples

Example 3.5. In Figure 3.31(a), a parameter 'searchTerm' has been introduced to the 'Search' action. The Parameter class of the PCM enables specifying a name and a data type (not shown here) for a parameter. Thus, it includes only information about the formal parameter. The actual parameter, i.e., the value a parameter takes when the service is actually called, can be characterised with a VariableUsage. In this case, the parameter is a string, which is the name of the item to be searched for. The database is assumed to contain 40 items. The domain expert has characterised the value of the input parameter and has specified a probability distribution for the search terms users pass to the service. Therefore, the domain expert has divided the input domain of the service into four subdomains (item1-10, item11-20, item21-30, item31-40) to reduce the modelling effort, and has provided probabilities for each of these subdomains. If the behaviour of the component service changes depending on which item is searched for (e.g., because of calling different databases), this can be included in the performance prediction, because the parameter has been characterised.

Example 3.6. In Figure 3.31(b), an array 'listOfItems' is passed to the 'BuyItems' action. The domain expert has not characterised the value of this array, but just the number of elements it contains. It is a suitable abstraction of the parameter in this case, because the value of the array is not relevant in this example. The service 'Buy Items' calls required services for each item in the array (not shown here because this is part of the service's SEFF and not the usage model), so that the number of elements in the array is sufficient for the performance predictions, as it is directly related to the number of loop iterations 
in the SEFF of this service. The number of elements is specified as a probability distribution, so that the loop is iterated with the same probability distribution.

\subsubsection{Structure}

The PCM parameter model (Figure 3.32) allows characterising actual parameters of a component service by associating a VariableUsage with a formal Parameter. The formal Parameter is part of an interface from the repository model (see Section 3.1.2) and referenced from the parameter model using an AbstractNamedReference. This may be a NamespaceReference or a concrete VariableReference, which contains the name of the parameter to be characterised. With NamespaceReferences more complex data structures such as composite data types or the inner elements of collections can be referenced. For example, an object 'customer' containing two strings 'name' and 'address', can be characterised by providing characterisations for both 'customer.name' and 'customer.address'.

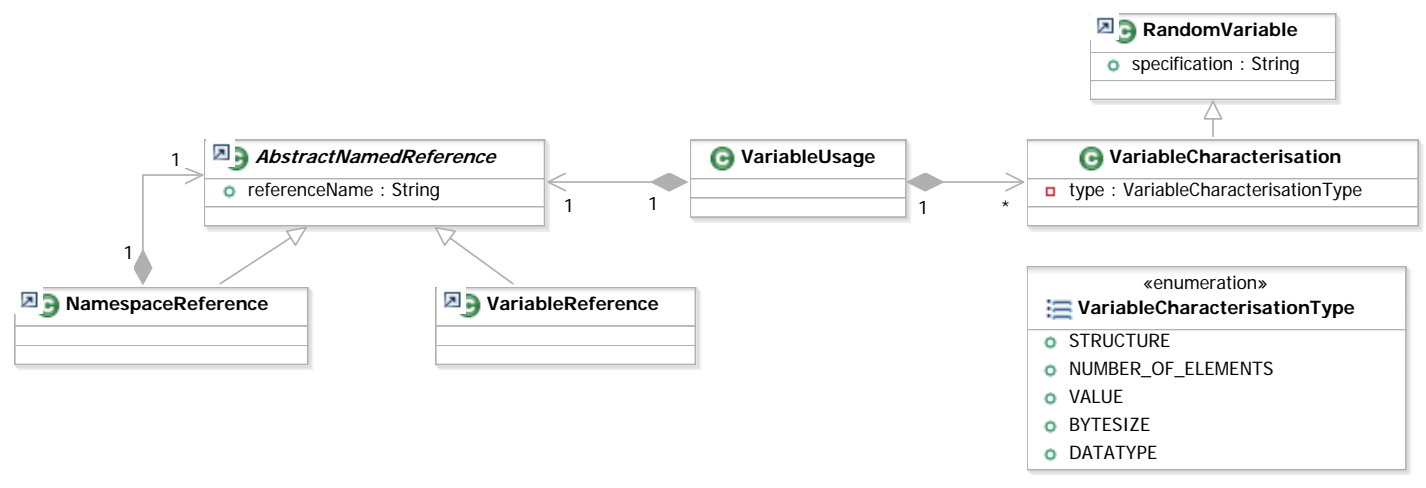

Figure 3.32: Parameter Model

Note, that it is only necessary to characterise parameters if they indeed influence performance or reliability. Many parameters do not change resource usage or alter the control flow between components, and their characterisation can be omitted. Characterising every parameter of the services in a complex component-based architecture would require too much effort and not support performance analysis.

Many parameters can be characterised by simply providing a constant value for them. However, as motivated in the example above, in some situations it is useful to characterise parameters not only with constant values but with probability distributions to allow more fine-grained predictions. Thus the attributes of parameters are characterised with VariableCharacterisation in the PCM, which inherit from RandomVariables (see Section 2.5).

Different attributes of parameters can be characterised in the PCM parameter model. Primitive data types can be characterised with their value, byte size, or data type. To demonstrate the modelling capabilities, consider the examples for primitive parameters in Figure 3.33. Note, that these examples are illustrated with class diagrams instead of the annotated activities used before.

Example 3.7. In Figure 3.33(a), a probability distribution for the value of the integer parameter named "id" has been specified. The parameter receives the values 1, 2, or 3 with probabilities of $70 \%, 20 \%$, and $10 \%$ respectively. Figure 3.33(b) shows a parameter named "inputFile", whose size in bytes has be specified as a constant value (20). Via inheritance, extensions of certain parameters may be passed to a component service, thus the concrete data type may additionally be characterised by a domain experts. 
In the example in Figure 3.33(c), the parameter "shape" of type GraphicObject may become a circle, triangle, or rectangle, which may alter the response time of the service that is supposed to draw these graphics. It is also possible to specify multiple characterisations of a single parameters, for example to specify the value and byte size.

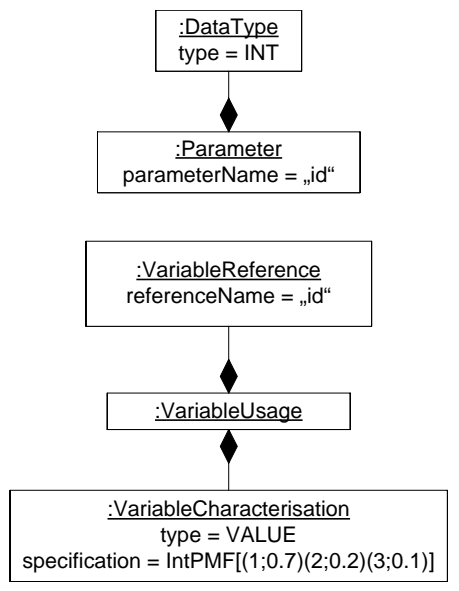

(a)

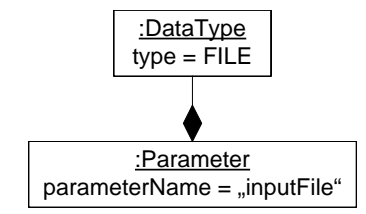

:VariableReference

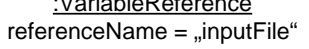

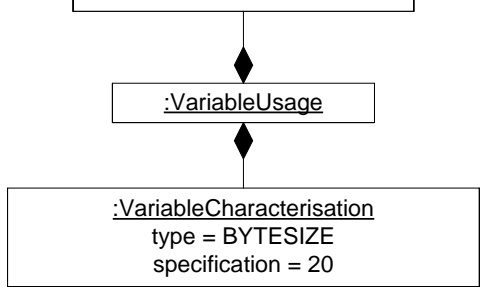

(b)

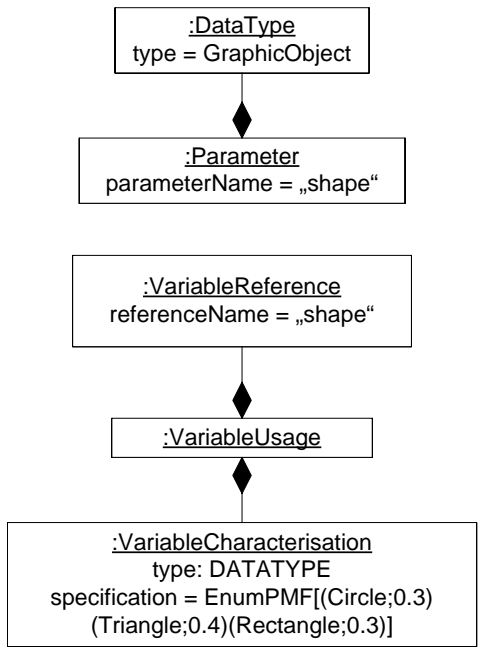

(c)

Figure 3.33: Primitive Parameter Characterisation Examples

Example 3.8. For collection parameters, it is more difficult to characterise the value domain. The performance-influence of collections like array, tree, or hash can sometimes be characterised simply by the number of elements. Thus, it may be appropriate for such parameters to specify probability distributions over the number of elements. Consider the example in Figure 3.34(a): the number of elements in the collection "niceTree" of type RedBlackTree has been specified with a probability distribution, i.e., the tree contains 10,100, or 1000 nodes with probabilities of $10 \%, 30 \%$, and $60 \%$. The value, byte size or data type of a collection can be characterised as explained above. In Figure 3.34(a), the size of the collection has additionally been specified.

Besides the number of elements, it is sometimes useful to specify the structure of a collection, if it influences QoS properties of a component service. For example presorted arrays may by sorted quicker than unsorted arrays or the deletion duration of an element in a tree may depend on the balance of the tree. In Figure 3.34(b), the structure of the array list "luckyNumbers" has been characterised as sorted with a probability of $10 \%$ and unsorted with a probability of $90 \%$. Additionally, the number of elements in the array list has been characterised with the constant value of 10.

To ease modelling, collection contain may contain one inner VariableUsage, which shall representatively model the inner elements of a collection. In the example in Figure 3.34(c), the collection "interestingFiles" is characterised with its number of elements. Additionally, the inner parameter usage representatively characterises a single file within the collection. Here, the files in the collection have a size in bytes between 10 and 40 bytes. 


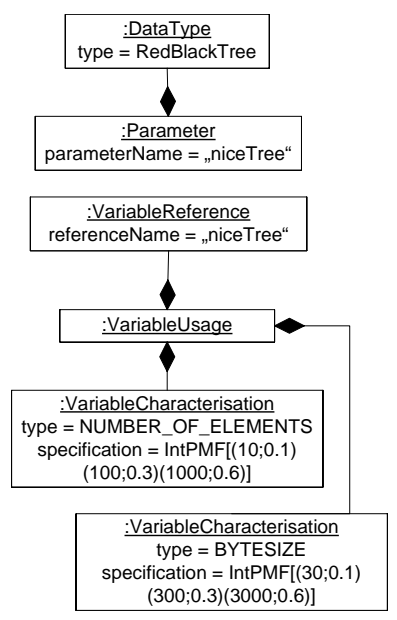

(a)

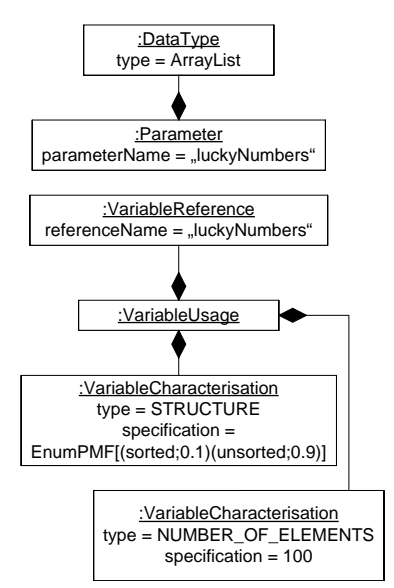

(b)

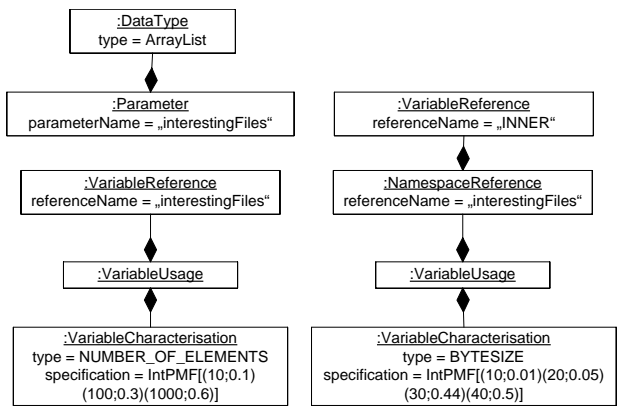

(c)

Figure 3.34: Collection Parameter Characterisation Examples

\subsubsection{Related Work}

Many performance prediction approaches or performance related meta models neglect the influence of parameters values to the above described properties. The UML SPT profile [31] as well as the CSM [32] do not include notions of parameters. Methods that build on these modelling approaches such as CB-SPE [35] thus also cannot express the influence of parameters to QoS properties.

KLAPER [33] allows characterising parameters values, but does not include a formal way of creating abstractions for parameters, because the kind of parameter specification is left open for developers. This limits the use of tools evaluating KLAPER instances, because they can not foresee all possible ways of abstracting parameters. Thus, manual work is required with KLAPER to complete the performance prediction process if parameters are involved.

The ROBOCOP component model [36] also allows characterising parameter values. However, as ROBOCOP aims at embedded systems, it is assumed that the domain for parameter values is very limited. It is possible to model parameters with constant values only, stochastical characterisation for parameter abstractions are not in the scope of that work.

The performance prediction approach by Hamlet et. al. [25] models components as functions and divides their input space into several subdomains. For each subdomain, which can be conceived as a parameter abstraction, different execution times can be determined. However, subdomains are always built for the values of parameters in this approach, other attributes of parameters are neglected. 


\subsection{QoS Analyst}

QoS analysts collect and integrate information from the other roles, extract QoS information from the requirements (e.g., maximal response times for use cases), and perform QoS analyses by using mathematical models or simulation. Furthermore, QoS analysts estimate missing values that are not provided by the other roles. For example, in case of an incomplete component specification, the resource demand of this component has to be estimated. Finally, they assist the software architects to interpret the results of the QoS analyses.

We have planned several metamodel extensions to support QoS analysts. They should support adding required QoS values to model entities and also store the result of QoS predictions attached to corresponding model constructs. For example, the response time of an use case can be attached to a UsageScenario or the throughput of a resource to a ProcessingResourceSpecification. However, these modelling constructs have not been finalised and are subject to future work. So far, the QoS analyst is not explicitly supported by the PCM. 
Chapter 4

\section{Technical Reference}




\subsection{Core and Repository}

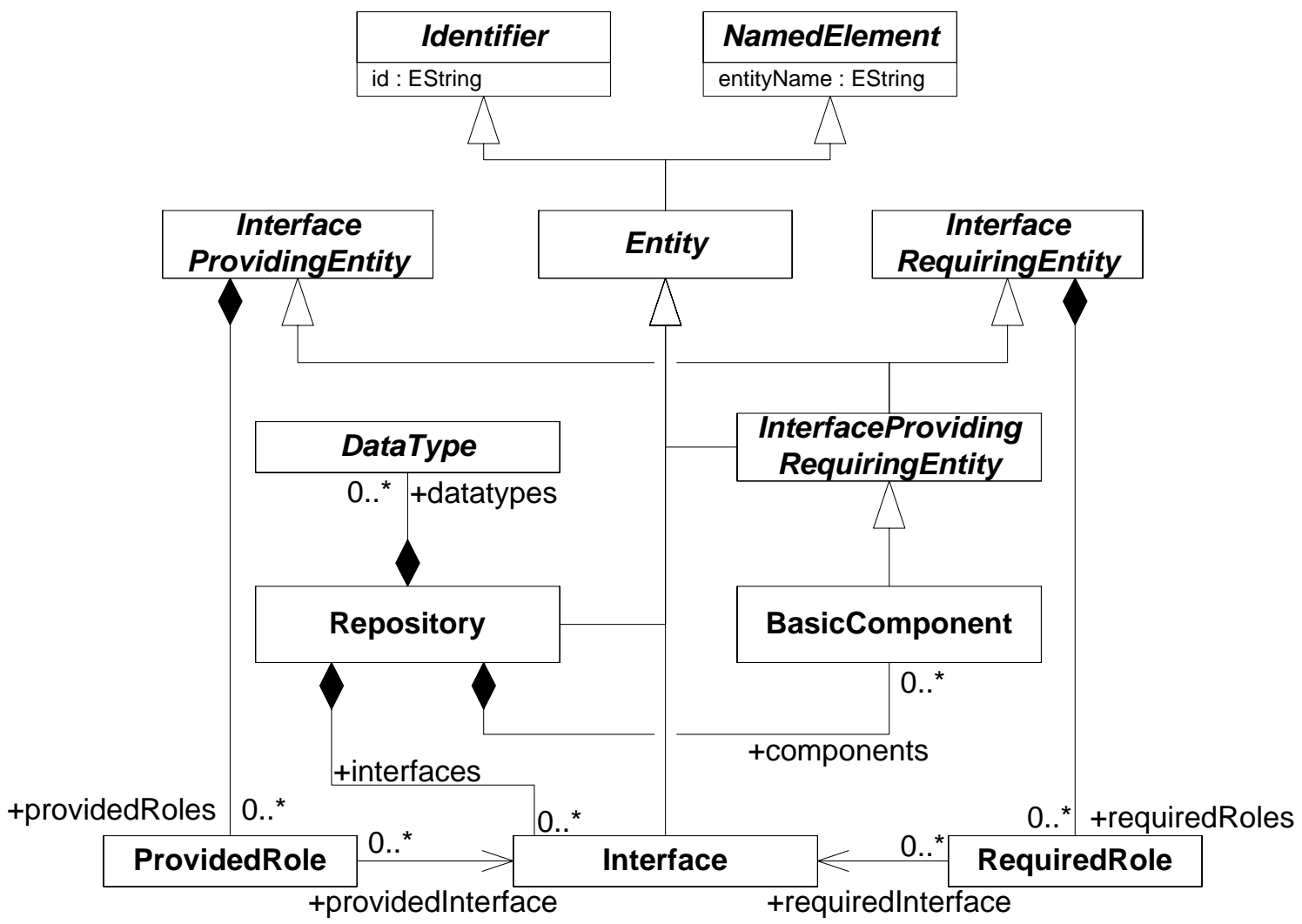

Figure 4.1: Repository 


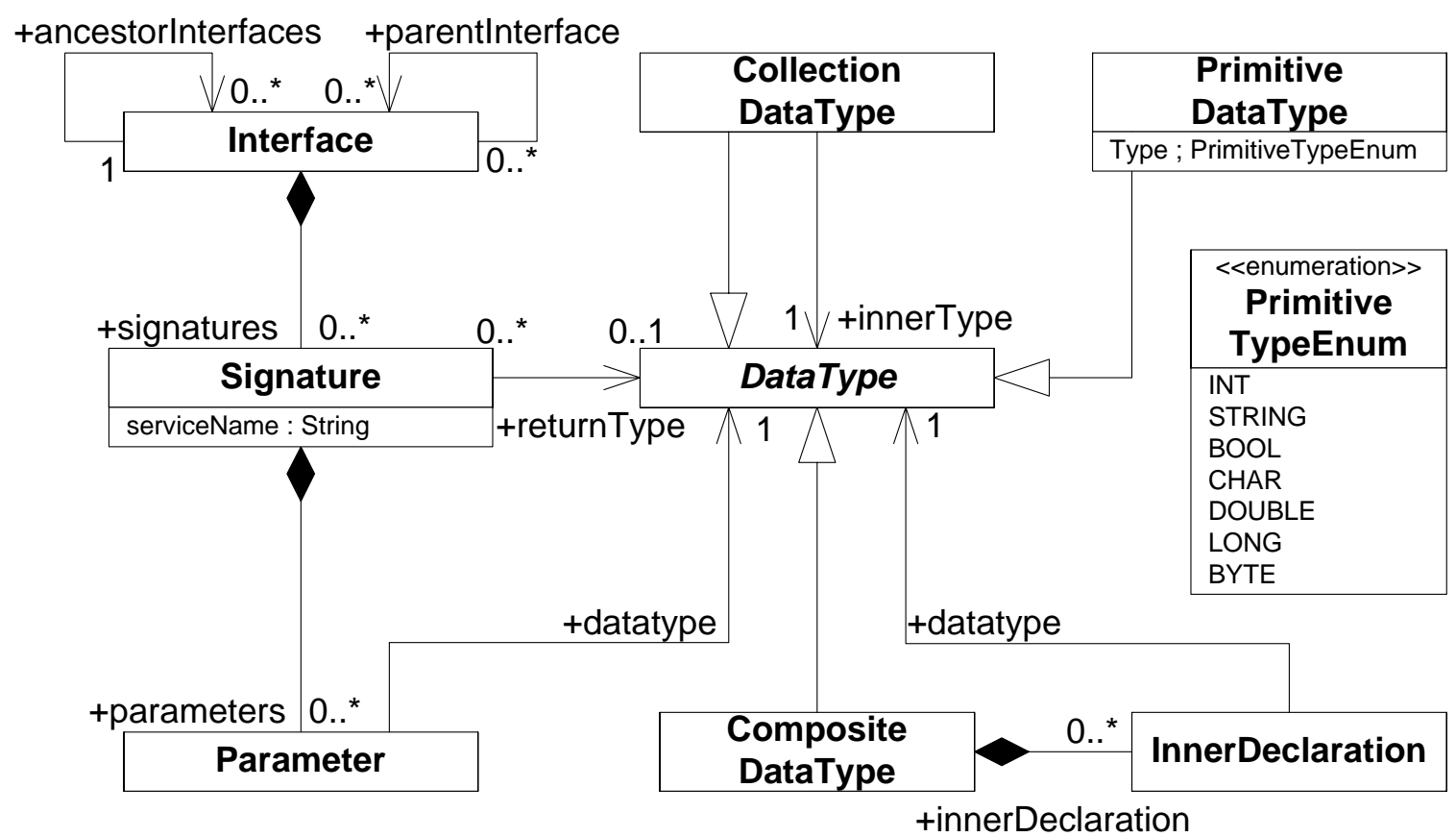

Figure 4.2: Interface

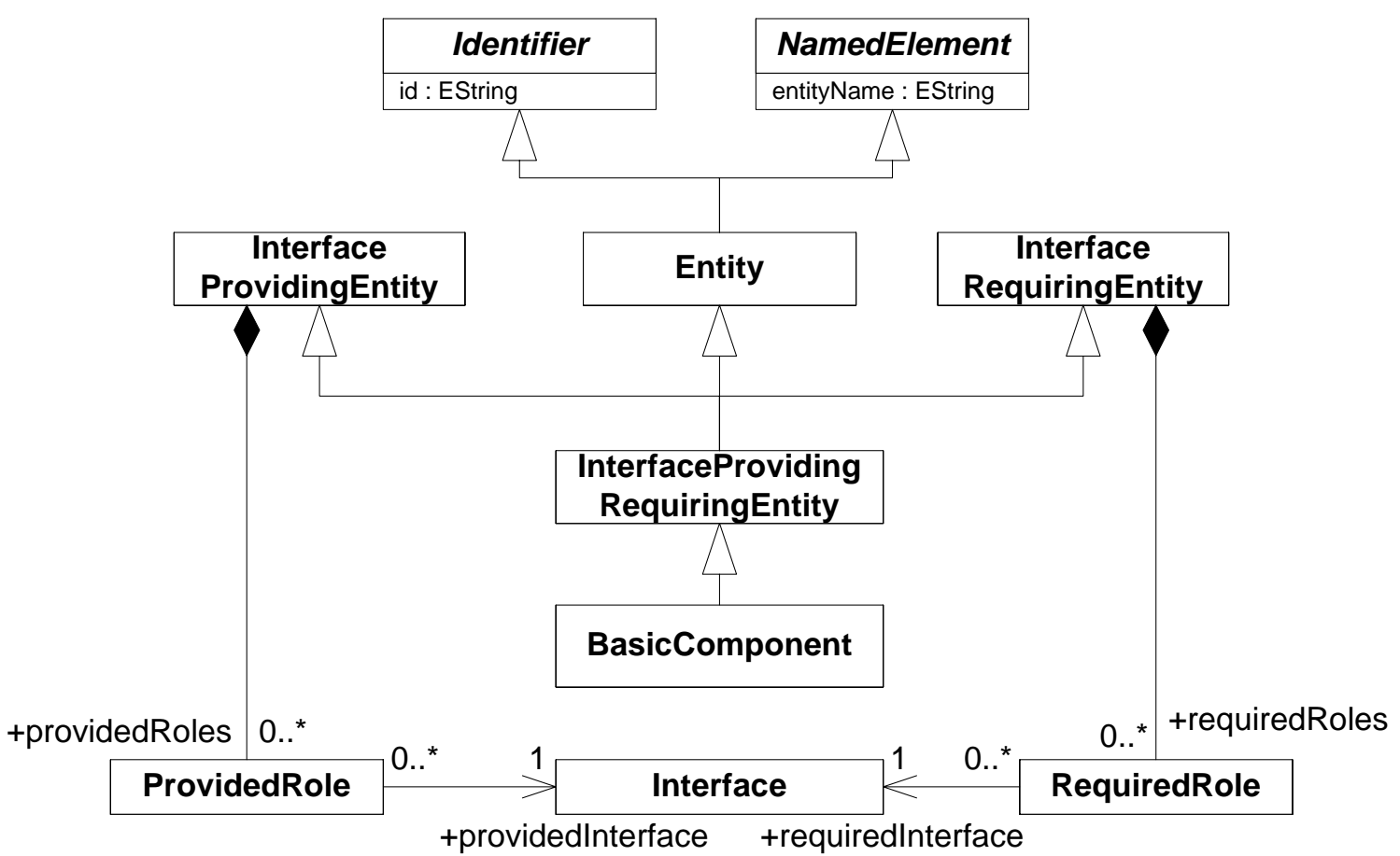

Figure 4.3: Component 


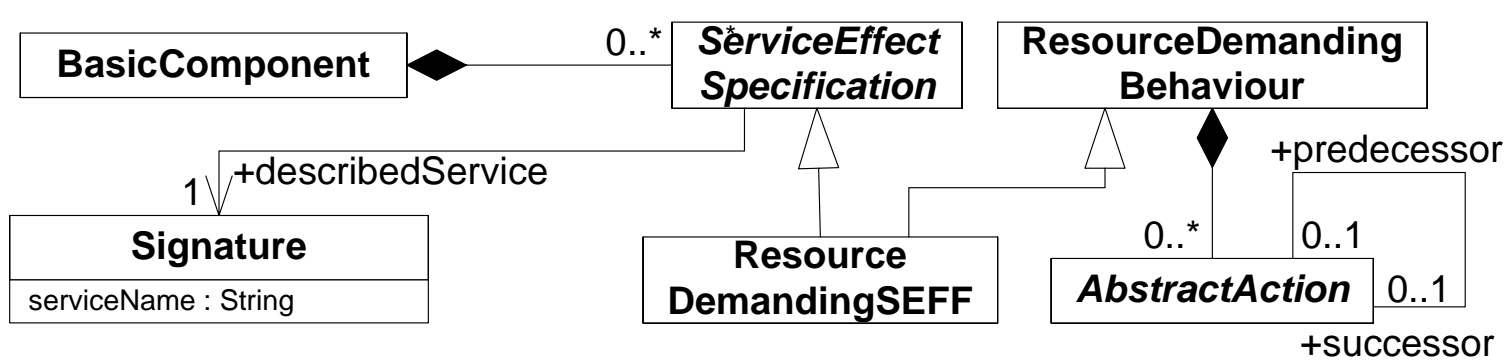

Figure 4.4: Resource Demanding Service Effect Specification (1/5)

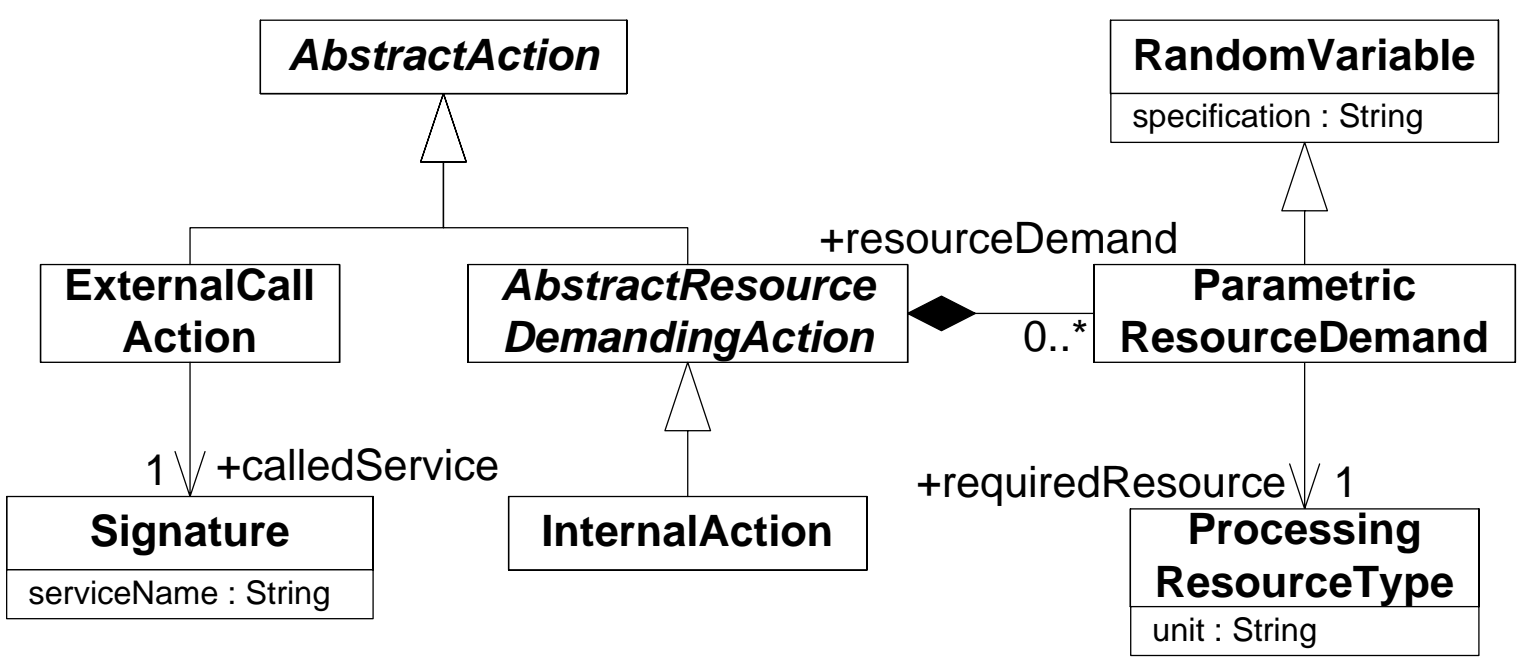

Figure 4.5: Resource Demanding Service Effect Specification (2/5)

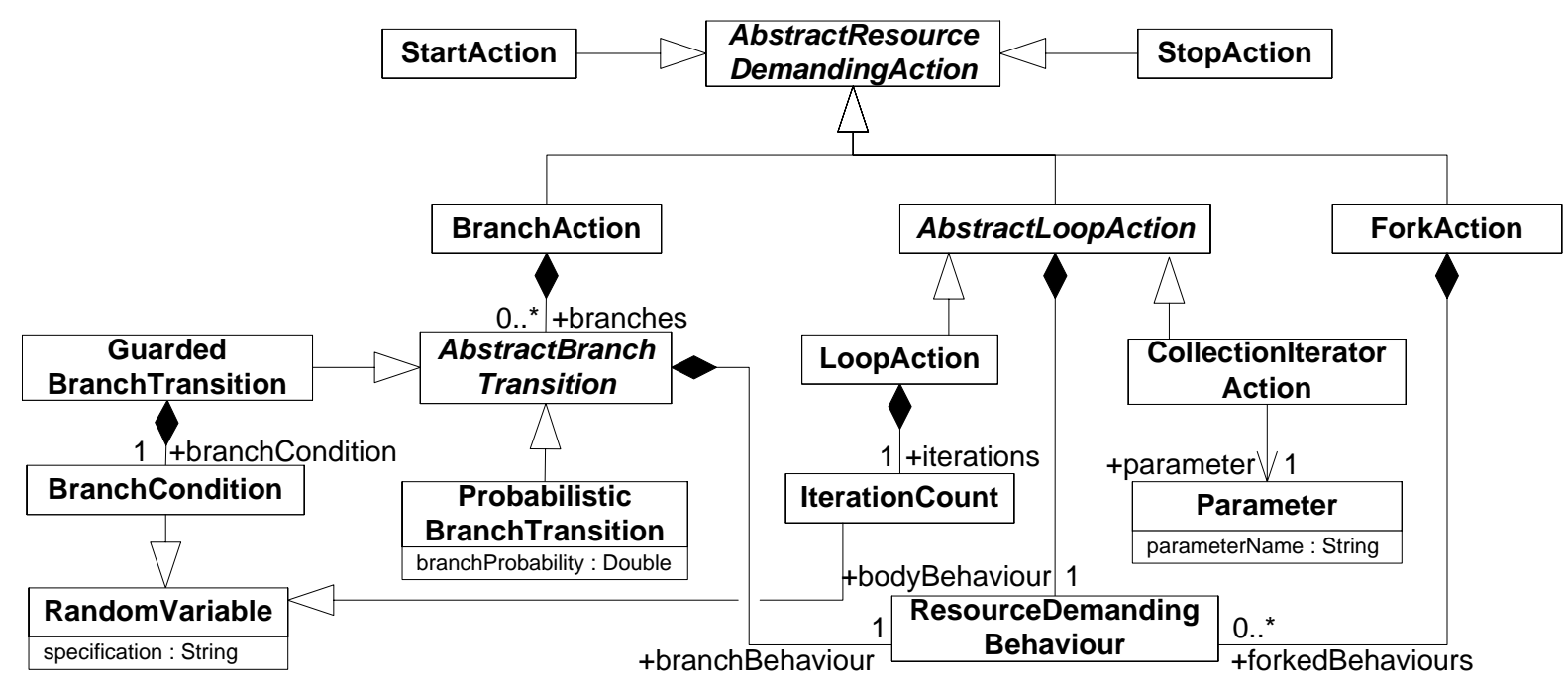

Figure 4.6: Resource Demanding Service Effect Specification (3/5) 


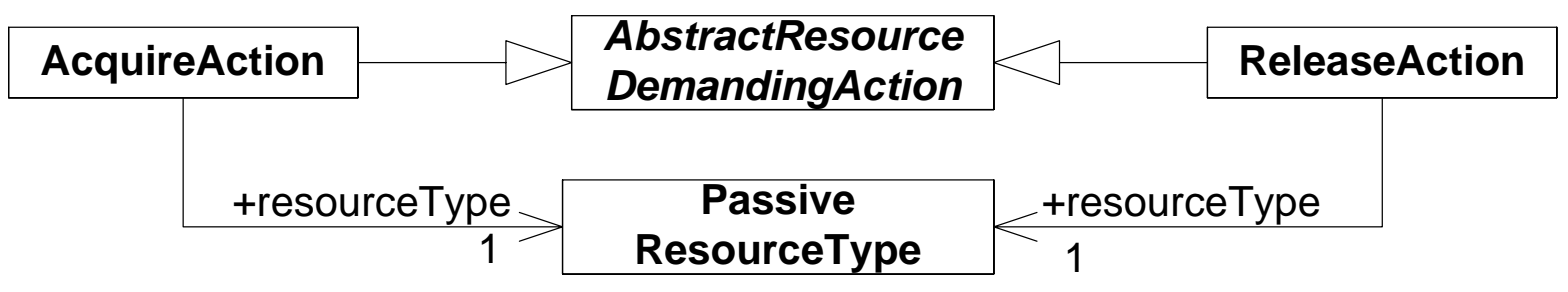

Figure 4.7: Resource Demanding Service Effect Specification (4/5)

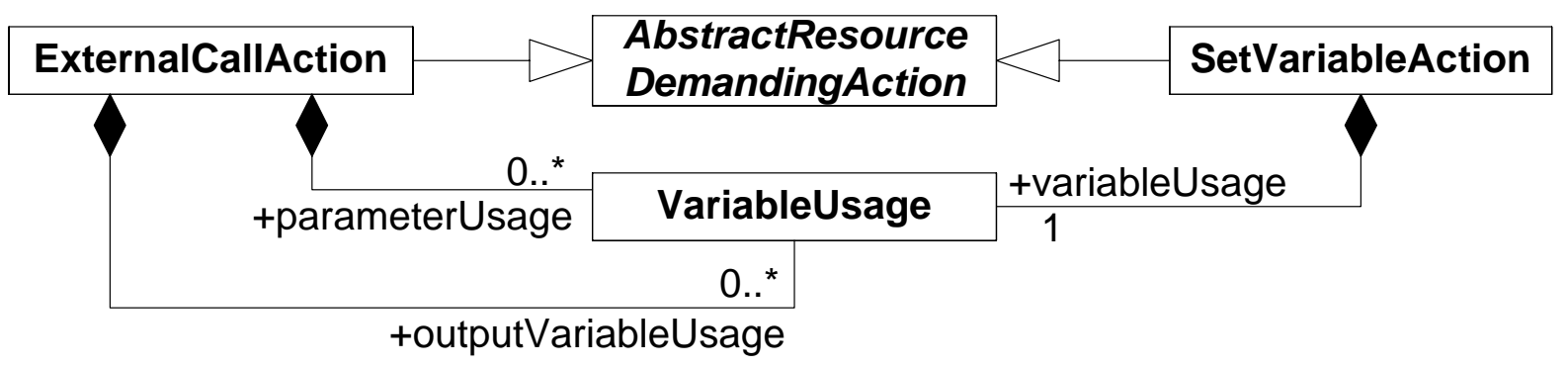

Figure 4.8: Resource Demanding Service Effect Specification (5/5) 


\subsection{Assembly and System}

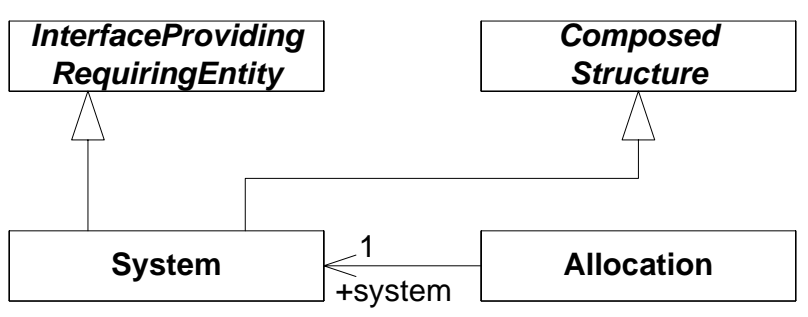

Figure 4.9: System

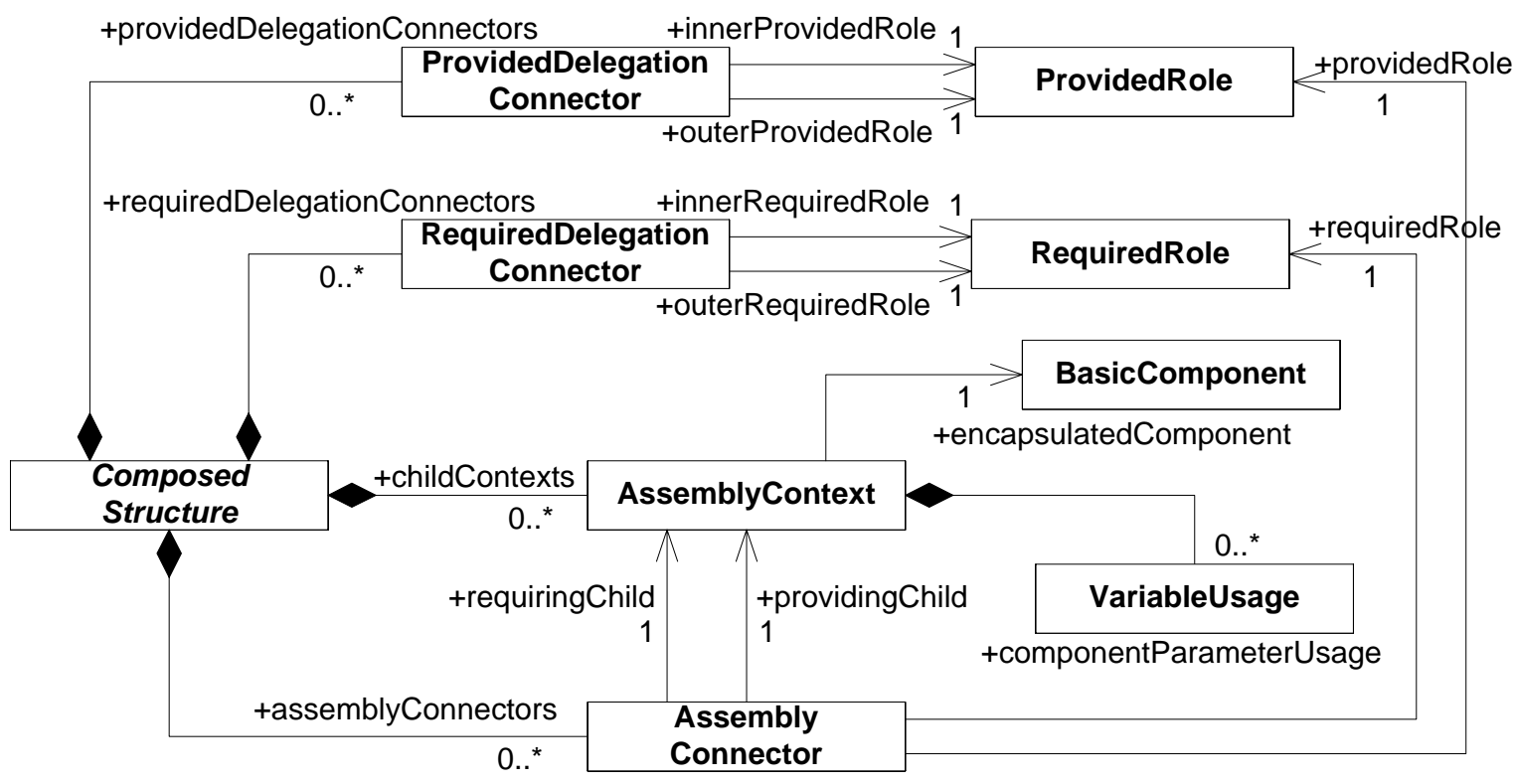

Figure 4.10: Composed Structure 


\subsection{Resource Type and Resource Environment}

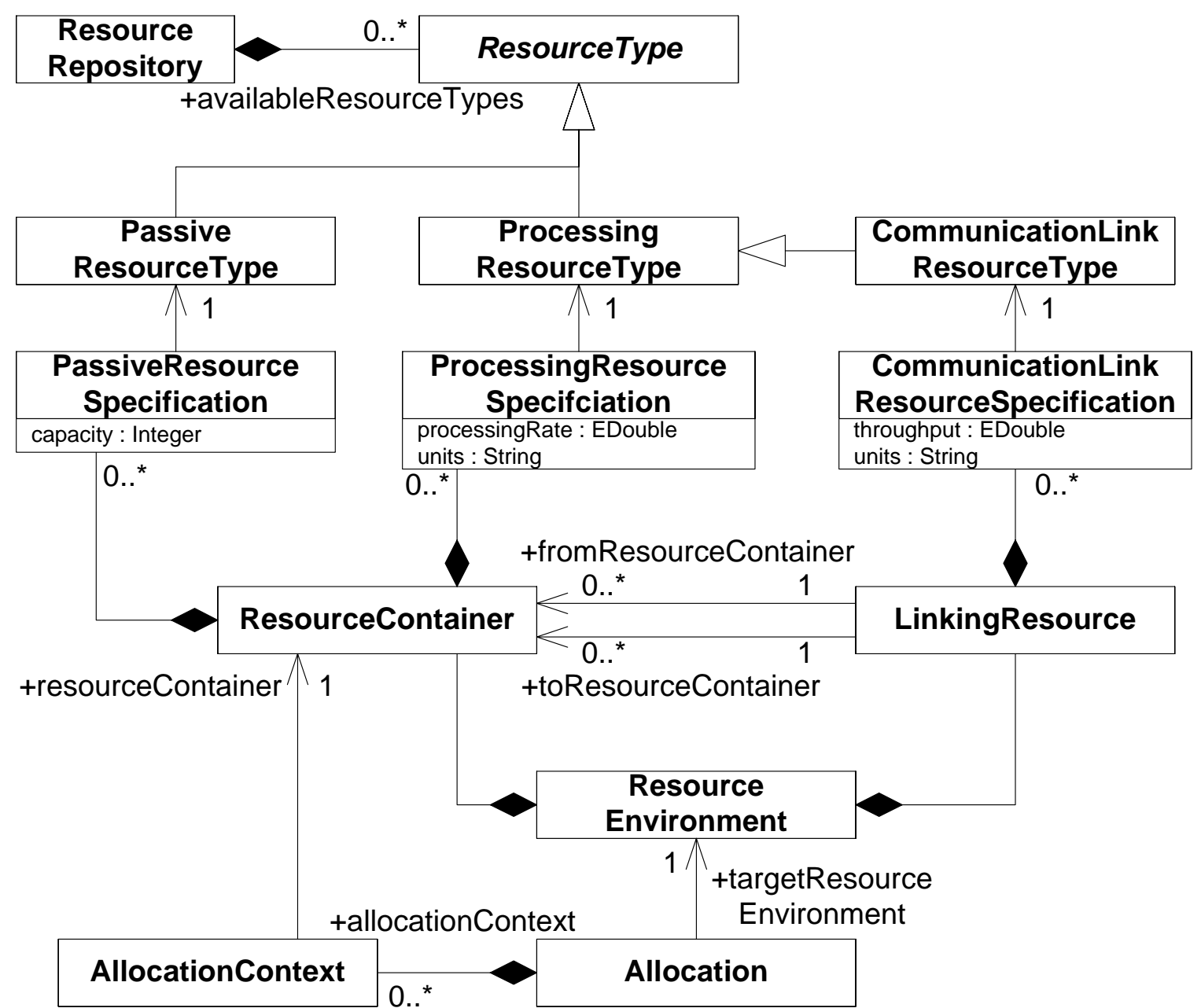

Figure 4.11: Resource Types and Resource Environment 


\subsection{Usage Model}

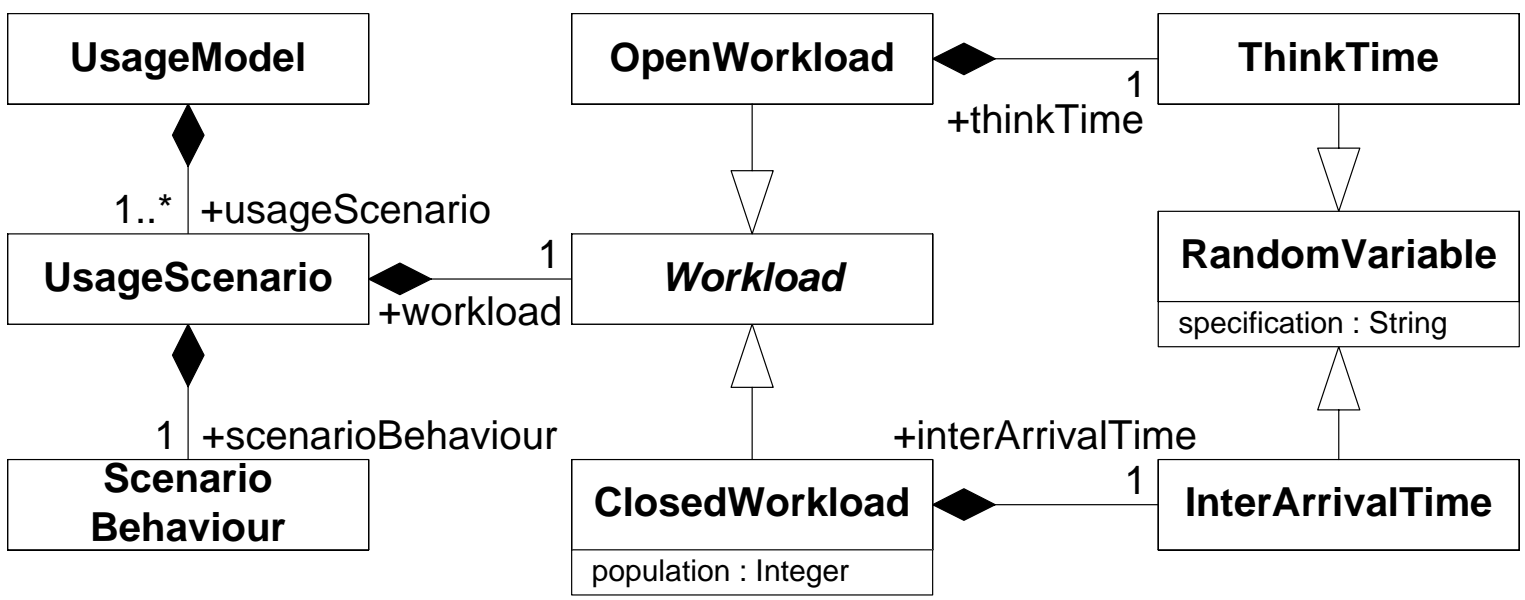

Figure 4.12: Usage Model 


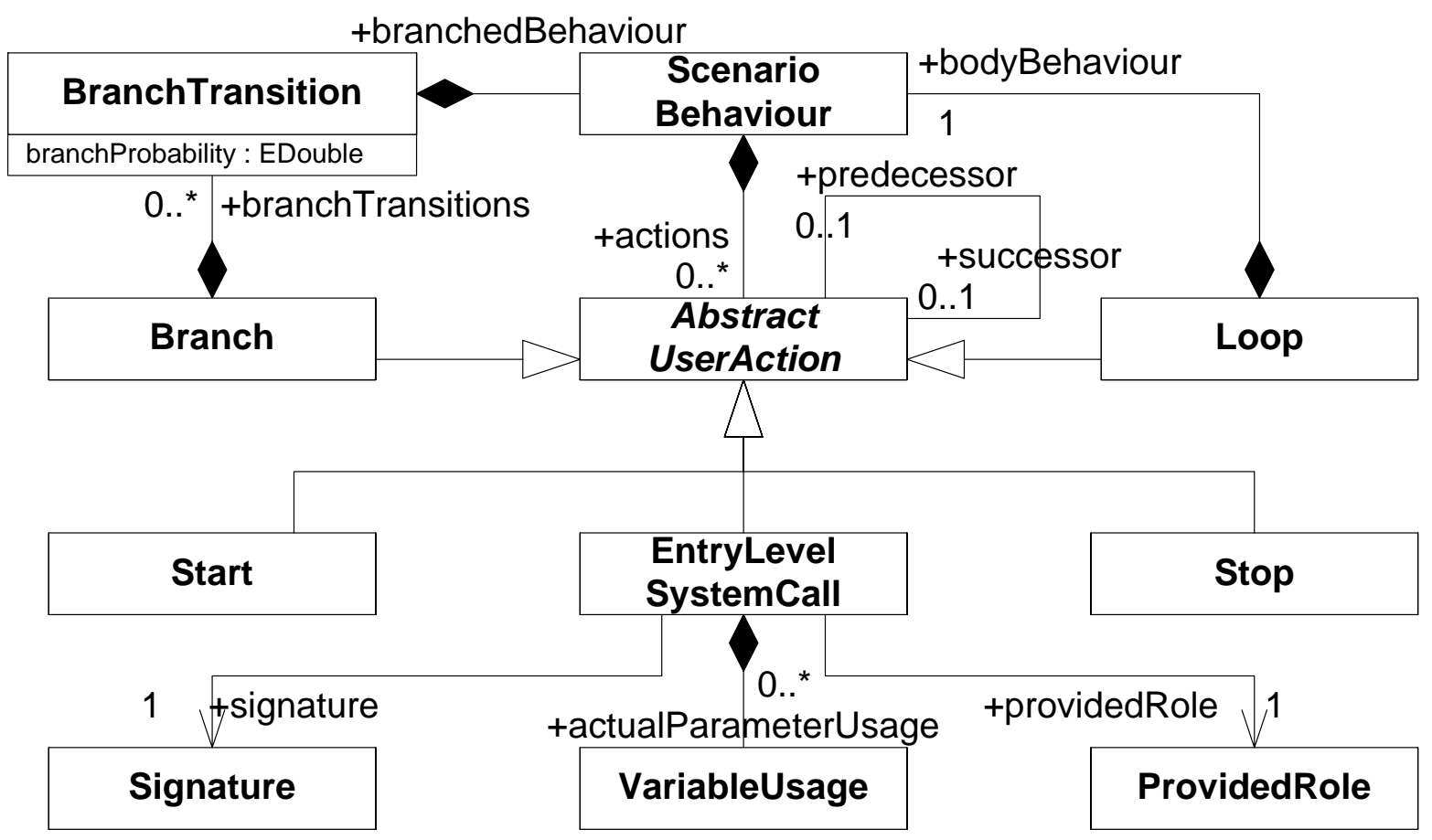

Figure 4.13: Usage Model Scenario Behaviour

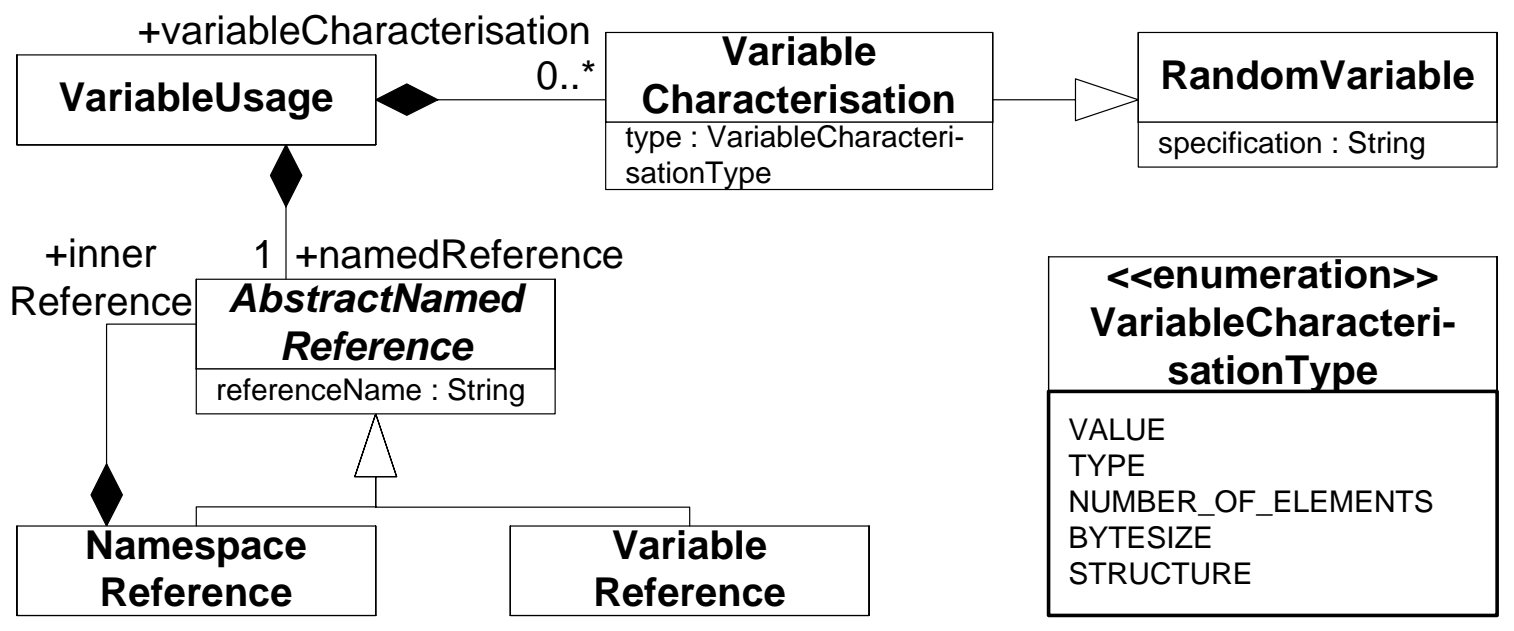

Figure 4.14: Variable Usage 
Chapter 5

Discussion 


\subsection{PCM versus UML2}

Despite the fact that several concepts in the PCM have counterparts in the UML2 meta-model, the PCM's design is intentionally not based on the UML2 meta-model. We argue against commonly used arguments for using UML2 and highlight its additional problems which hinder an approach as described in this paper.

Common arguments for using UML2 as foundation of performance prediction models are the widespread use and familiarity of the developers with UML2, the availability of model-instances and the reuse of existing concepts.

We agree that using a notation familiar to developers is necessary to increase the willingness to accept and use a new technique. As a consequence we reused the UML2 graphical notation whenever it appeared adequate. However, as we use model-driven techniques to transform the model instances into prediction models, the source model should be unambiguous in a way which ideally only has one concept to model a certain fact. In UML2 many constructs exist which allow modelling a single fact in many different ways. Take for example a loop modelled in an UML2 activity diagram. Either an iterator node or a control flow going backwards may be used to express it. Facing such ambiguities, transformations turn out to be overly complex as they have to identify all these different options.

The same argument also holds for the reuse of existing UML2 models for performance predictions. Industrial style UML2 models have mostly been designed for human communication. Hence, they use models which need further explanations, UML2 notes, or additional documentation which renders them unsuited for automated, machine interpreted model transformations. Besides the effort of addition performance annotations using a UML profile, such models would need significant effort to prepare them for automated predictions aligning the model with the concepts as expected by the transformation (for further discussions on using UML2 in model-driven approaches see [37]).

Using UML2 tools and profiles for performance annotations raises an additional issue. Performance annotations like those defined in the UML-SPT profile are rather complex and their attachment to model elements is error-prone. Support for this task by means of modern UIs, like on the fly error correction, syntax highlighting, etc. is crucial. However, the UML stereotype mechanism allows only for editing basic datatypes like strings or numbers with very basic editing capabilities. As a way out, some UML tools offer extension mechanisms to customise the tool's editing capabilities. However, such extensions need special coding for every UML2 tool available. In addition to the issues with specifying the annotations, additional problems arise when implementing model transformations using standard transformation approaches like QVT. In present state, support for stereotypes is limited as they do not offer any means to parse tagged values. For SPT based annotations this means transforming the annotations using for example ad-hoc Java transformations which means loosing the advantage of the standardised transformation engine again.

Reusing UML2 concepts sounds good on first sight as well. In our PCM, several concepts like interface, signature, etc. have their counterparts in UML2. However, as UML2 is a large and complex meta-model respecting all kinds of concepts available is difficult - especially, when defining the concept's meaning wrt. a performance prediction model. Therefore, the PCM is restricted to concepts for which we know how to map them onto the performance domain and how to predict their performance impact.

Finally, UML2 is not designed specifically for the aim of doing performance predictions which can be seen by the need of profiles for model annotations. Opposed to that, the PCM includes advanced concepts coming from the CBSE as well as from the performance domain. Examples of the additional concepts are service effect specifications, a component type hierarchy, inherent support for performance annotations and an explicit component context model for expressing a components QoS in dependence 
of its environment [38]. In this paper, we focus on QoS-relevant modelling elements, a specification of the other concepts can be found on the PCM's website [39].

With all that said, it becomes clear that using the PCM for legacy projects includes migrating existing UML2 model into the PCM. If the models have been designed for human communication, this might not be a problem as the models need checking anyhow. For models which have already been designed for model driven approaches, writing a transformation to initially transform the UML2 model into the PCM becomes necessary. 


\subsection{Related Work}

Component Models In recent years, many component models have been developed for many different purposes. A taxonomy of these models can be found in [7].

- ROBOCOP: [40] allows performance predictions, aims at embedded systems.

- PACC: [41] allows performance predictions, aims at embedded systems.

- Koala: [42] no QoS, aims at embedded systems

- SOFA: [21] allows protocol checking.

- Fractal: allows runtime reconfigurations of architectures

- UML: [43] limited component concept

- CCM: no QoS

- EJB: no QoS

- COM+: no QoS

Performance Meta-Models To describe the performance properties of software systems, several metamodels have been introduced (Survey by [44]).

- SPE-Metamodel: [45] designed for object-oriented software systems.

- UML+SPT profile: [31] offers capabilities to extend UML models with performance properties. Is not suited for parametric dependencies, which are needed for component specifications.

- CSM: [32] is closely aligned with the UML-SPT approach and does not target component-based systems.

- KLAPER: [33] is designed for component-based architectures and reduces modelling complexity by treating components and resources in a unified way. 


\subsection{Open Issues and Limitations}

- Resource Model: The PCM's resource model is still very limited and supports only a few types of abstract resource types on the hardware layer. QoS influencing factors from the middleware, the virtual machine, and operating system are still missing in the PCM's resource model. We plan to introduce a layered resource model, where different system levels (such as middleware, operating system, hardware) can be modelled independently and then composed vertically, just as software components are composed horizontally.

- Dynamic Architectures: The PCM is only targeted at static architectures, and does not allow the creation/deletion of components during runtime or changing links between components. With the advent of web services and systems with dynamic architectures changing during runtime, researchers pursuit methods to predict the dynamic performance properties of such systems.

- Prediction Result Interpretation and Feedback: While today's model-transformations in software performance engineering bridge the semantic gap from the developer-oriented models to the analytical models, the opposite direction of interpreting performance result back from the analytical models to the developer-oriented models has received sparse attention. Analytical performance results tend to be hard to interpret by developers, who lack knowledge about the underlying formalisms. Thus, an intuitive feedback from the analytical models to the developer-oriented models would be appreciated.

- State of Component Protocols: Parametric contracts model dependencies between component interfaces with protocols which, therefore, have a state. In some cases, this leads to difficulties when analysing the interoperability of components communicating via an interface. Assume an interface provided by component $A$ is accessed by components $B$ and $C$. If $B$ changes the state of the interfaces by calling a service, does component $C$ see the changes or does it have its own view on $A$ ? This question cannot be answered in general. In some cases, components share the state, e.g. when using the Singleton pattern, in other cases they don't. To solve this issue, additional information in the component model is required. Ports and interfaces with cardinalities seem to be a promising concept.

- Identification of the Relevant QoS Parameters: To achieve accurate QoS predictions, the parameters influencing the attributes of interest need to be identified. A lot of work has already been done in this context, in UML for example by the definition of the UML SPT profile [46]. However, the existing work needs to be reviewed, to be extended and the identified parameters needed to be specified within our component model. Furthermore, means to analyse and derive the desired performance metrics from the input values have to be found and/or developed.

- High-level concurrency modelling constructs: We plan to add special modelling constructs for concurrent control flow into the PCM. This shall relieve the burden from developers to specify concurrent behaviour with basic constructs, such as forks or semaphores for synchronisation. The concurrency modelling constructs shall be aligned with known concurrency patterns and be configurable via feature diagrams. Model-transformations shall transform the high-level modelling constructs to the performance domain of analytical and simulation models. 


\section{Bibliography}

[1] Steffen Becker, Heiko Koziolek, and Ralf Reussner, "Model-based Performance Prediction with the Palladio Component Model," in Proceedings of the 6th International Workshop on Software and Performance (WOSP2007). February5-8 2007, ACM Sigsoft.

[2] Heiko Koziolek and Jens Happe, “A Quality of Service Driven Development Process Model for Component-based Software Systems," in Component-Based Software Engineering, Ian Gorton, George T. Heineman, Ivica Crnkovic, Heinz W. Schmidt, Judith A. Stafford, Clemens A. Szyperski, and Kurt C. Wallnau, Eds. July 2006, vol. 4063 of Lecture Notes in Computer Science, pp. 336-343, Springer-Verlag GmbH.

[3] Heiko Koziolek, Jens Happe, and Steffen Becker, "Parameter dependent performance specification of software components," in Proceedings of the Second International Conference on Quality of Software Architectures (QoSA2006). July 2006, vol. 4214 of Lecture Notes in Computer Science, pp. 163-179, Springer-Verlag, Berlin, Germany.

[4] Viktoria Firus, Steffen Becker, and Jens Happe, "Parametric Performance Contracts for QMLspecified Software Components," in Formal Foundations of Embedded Software and Componentbased Software Architectures (FESCA). 2005, vol. 141 of Electronic Notes in Theoretical Computer Science, pp. 73-90, ETAPS 2005.

[5] Ralf H. Reussner, Heinz W. Schmidt, and Iman Poernomo, "Reliability prediction for componentbased software architectures," Journal of Systems and Software - Special Issue of Software Architecture - Engineering Quality Attributes, vol. 66, no. 3, pp. 241-252, 2003.

[6] Clemens Szyperski, Dominik Gruntz, and Stephan Murer, Component Software: Beyond ObjectOriented Programming, ACM Press and Addison-Wesley, New York, NY, 2 edition, 2002.

[7] K.-K. Lau and Z. Wang, "A Taxonomy of Software Component Models," in Proceedings of the 31st EUROMICRO Conference. 2005, pp. 88-95, IEEE Computer Society Press.

[8] Simonetta Balsamo, Antinisca Di Marco, Paola Inverardi, and Marta Simeoni, "Model-Based Performance Prediction in Software Development: A Survey," IEEE Transactions on Software Engineering, vol. 30, no. 5, pp. 295-310, May 2004.

[9] Katerina Goseva-Popstojanova and Kishor S. Trivedi, "Architecture-based approach to reliability assessment of software systems," Performance Evaluation, vol. 45, no. 2-3, pp. 179-204, 2001.

[10] John Cheeseman and John Daniels, UML Components: A Simple Process for Specifying Component-based Software, Addison-Wesley, Reading, MA, USA, 2000. 
[11] Steffen Becker, Lars Grunske, Raffaela Mirandola, and Sven Overhage, "Performance Prediction of Component-Based Systems: A Survey from an Engineering Perspective," in Architecting Systems with Trustworthy Components, Ralf Reussner, Judith Stafford, and Clemens Szyperski, Eds., vol. 3938 of LNCS, pp. 169-192. Springer, 2006.

[12] Ralf H. Reussner, Parametrisierte Verträge zur Protokolladaption bei Software-Komponenten, Dissertation, Fakultät für Informatik, Universität Karlsruhe (TH), Germany, July 2001.

[13] Jim Q. Ning, “A component-based software development model," in COMPSAC '96: Proceedings of the 20th Conference on Computer Software and Applications, Washington, DC, USA, 1996, p. 389, IEEE Computer Society.

[14] A. W. Brown and K. C. Wallnan, "Engineering of component-based systems," in ICECCS '96: Proceedings of the 2nd IEEE International Conference on Engineering of Complex Computer Systems (ICECCS '96), Washington, DC, USA, 1996, p. 414, IEEE Computer Society.

[15] E. Teiniker, G. Schmoelzer, J. Faschingbauer, C. Kreiner, and R. Weiss, "A hybrid componentbased system development process," in Proceedings of 31st EUROMICRO Conference on Software Engineering and Advanced Applications, August 2005, pp. 152-159.

[16] Ivica Crnkovic, Michel Chaudron, and Stig Larsson, "Component-based development process and component lifecycle," in International Conference on Software Engineering Advances, ICSEA'06, Tahiti, French Polynesia, October 2006, IEEE.

[17] David Lorge Parnas, "On the criteria to be used in decomposing systems into modules," Сотmunications of the ACM, vol. 15, no. 12, pp. 1053-1058, Dec. 1972.

[18] Bertrand Meyer, Object-Oriented Software Construction, Prentice Hall, Englewood Cliffs, NJ, USA, 2 edition, 1997.

[19] A. V. Aho and J. D. Ullman, Foundations of Computer Science, Computer Science Press, W.H. Freeman and Co., New York, 1992.

[20] Ralf H. Reussner, "Automatic Component Protocol Adaptation with the CoCoNut Tool Suite," Future Generation Computer Systems, vol. 19, pp. 627-639, July 2003.

[21] Frantisek Plasil and Stanislav Visnovsky, "Behavior Protocols for Software Components," IEEE Transactions on Software Engineering, vol. 28, no. 11, pp. 1056-1076, 2002.

[22] Ralf H. Reussner, Steffen Becker, and Viktoria Firus, "Component Composition with Parametric Contracts," in Tagungsband der Net.ObjectDays 2004, 2004, pp. 155-169.

[23] Franz Eisenführ and Martin Weber, Rationales Entscheiden, Springer Verlag, Berlin, u.a., 4. edition, 2003.

[24] Object Management Group, "Common Object Request Broker Architecture: Core Specification," March 2004, [letztes Abrufdatum 29.04.2006].

[25] Dick Hamlet, Dave Mason, and Denise Woit, Component-Based Software Development: Case Studies, vol. 1 of Series on Component-Based Software Development, chapter Properties of Software Systems Synthesized from Components, pp. 129-159, World Scientific Publishing Company, March 2004. 
[26] Jens Happe and Viktoria Firus, "Using stochastic petri nets to predict quality of service attributes of component-based software architectures," in Proceedings of the Tenth Workshop on Component Oriented Programming (WCOP2005), 2005.

[27] Heiko Koziolek and Viktoria Firus, "Parametric Performance Contracts: Non-Markovian Loop Modelling and an Experimental Evaluation," in Proceedings of FESCA2006, 2006, Electronical Notes in Computer Science (ENTCS).

[28] Douglas Schmidt, Michael Stal, Hans Rohnert, and Frank Buschmann, Pattern-Oriented Software Architecture - Volume 2 - Patterns for Concurrent and Networked Objects, Wiley \& Sons, New York, NY, USA, 2000.

[29] E.D. Lazowska, J. Zahorjan, G. S. Graham, and K. C. Sevcik, Quantitative System Performance Computer System Analysis Using Queueing Network Models, Prentice-Hall, 1984.

[30] Holger Hermanns, Ulrich Herzog, and Joost-Pieter Katoen, "Process Algebra for Performance Evaluation," Theoretical Computer Science, vol. 274, no. 1-2, pp. 43-87, 2002.

[31] Object Management Group (OMG), "UML Profile for Schedulability, Performance and Time," http://www.omg.org/cgi-bin/doc?formal/2005-01-02, January 2005.

[32] Murray Woodside, Dorina C. Petriu, Hui Shen, Toqeer Israr, and Jose Merseguer, "Performance by unified model analysis (PUMA)," in WOSP '05: Proceedings of the 5th International Workshop on Software and Performance, New York, NY, USA, 2005, pp. 1-12, ACM Press.

[33] Vincenzo Grassi, Raffaela Mirandola, and Antonino Sabetta, "From Design to Analysis Models: a Kernel Language for Performance and Reliability Analysis of Component-based Systems," in WOSP '05: Proceedings of the 5th international workshop on Software and performance, New York, NY, USA, 2005, pp. 25-36, ACM Press.

[34] James A. Whittaker and Michael G. Thomason, "A Markov chain model for statistical software testing," IEEE Transactions on Software Engineering, vol. 20, no. 10, pp. 812-824, Oct. 1994.

[35] Antonia Bertolino and Raffaela Mirandola, "CB-SPE Tool: Putting Component-Based Performance Engineering into Practice," in Proc. 7th International Symposium on Component-Based Software Engineering (CBSE 2004), Edinburgh, UK, Ivica Crnkovic, Judith A. Stafford, Heinz W. Schmidt, and Kurt C. Wallnau, Eds. 2004, vol. 3054 of Lecture Notes in Computer Science, pp. 233-248, Springer.

[36] Egor Bondarev, Peter de With, Michel Chaudron, and Johan Musken, "Modelling of InputParameter Dependency for Performance Predictions of Component-Based Embedded Systems," in Proceedings of the 31th EUROMICRO Conference (EUROMICRO'05), 2005.

[37] Brian Henderson-Sellers, "UML - the good, the bad or the ugly? perspectives from a panel of experts," Software and System Modeling, vol. 4, no. 1, pp. 4-13, February 2005.

[38] Steffen Becker, Jens Happe, and Heiko Koziolek, "Putting components into context - supporting qos-predictions with an explicit context model," in Proceedings of the Eleventh International Workshop on Component-Oriented Programming (WCOP'06), Ralf Reussner, Clemens Szyperski, and Wolfgang Weck, Eds., June 2006. 
[39] Palladio, "The Palladio Component Model (Download and Documentation)," http: / / s dqweb . ipd.uka.de/wiki/Palladio_Component_Model, May 2007.

[40] Egor Bondarev, Peter H. N. de With, and Michel Chaudron, "Predicting Real-Time Properties of Component-Based Applications," in Proc. of RTCSA, 2004.

[41] Scott A. Hissam, Gabriel A. Moreno, Judith A. Stafford, and Kurt C. Wallnau, "Packaging Predictable Assembly.," in Component Deployment, IFIP/ACM Working Conference, CD 2002, Berlin, Germany, June 20-21, 2002, Proceedings, Judy M. Bishop, Ed. 2002, vol. 2370 of Lecture Notes in Computer Science, pp. 108-124, Springer.

[42] Rob van Ommering, Frank van der Linden, Jeff Kramer, and Jeff Magee, "The koala component model for consumer electronics software," Computer, vol. 33, no. 3, pp. 78-85, 2000.

[43] Object Management Group (OMG), "Unified modeling language specification: Version 2, revised final adopted specification (ptc/05-07-04)," 2005.

[44] Vittorio Cortellessa, "How far are we from the definition of a common software performance ontology?," in WOSP '05: Proceedings of the 5th International Workshop on Software and Performance, New York, NY, USA, 2005, pp. 195-204, ACM Press.

[45] C. U. Smith and L. G. Williams, Performance Solutions: A Practical Guide to Creating Responsive, Scalable Software, Addison-Wesley, 2002.

[46] Object Management Group (OMG), "UML Profile for Modeling Quality of Service and Fault Tolerance Characteristics and Mechanisms," http://www.omg.org/cgi-bin/doc?ptc/2005-05-02, May 2005. 


\section{Index}

Assembly, 59

Component Developer, 36

Connector

System Assembly Connector, 60

Context

Assembly Context, 59

Domain Expert, 69

External Calls, 51

Interface, 38

Parametric Dependencies, 55

Probability

PDF, 28

discetisized, 28, 29

PMF, 28

Protocol, 38

QoS Analyst, 78

Random Variable, 28

Definition, 28

Resource Demand, 50

Scenario Behaviour, 71

Service Effect Specification, 47

FSM, 47

RDSEFF Control Flow, 52

Resource Demanding SEFF, 49

Signature, 38

Software Architect, 58

System, 60

Delegation Connectors, 60

Roles, 60

Usage Model, 69

Workload, 71 